UNIVERSITÉ DU QUÉBEC A CHICOUTIMI

\author{
MÉMOIRE \\ PRÉSENTÉ À \\ UNIVERSITÉ DU QUÉBEC A CHICOUTIMI \\ COMME EXIGENCE PARTIELLE \\ DE LA MAITRISE EN SCIENCES APPLIQUÉES
}

\author{
PAR \\ RAYNALD RICHER \\ B.Sc. PHYSIQUE
}

ETUDE DE L'INTERACTION DES ONDES DE CHOC AVEC LA GLACE

À L'INTERFACE AIR-GLACE

Août 2003 


\section{Bibliothèque}

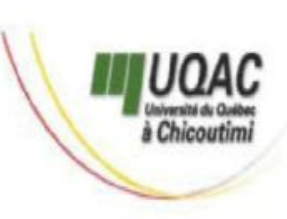

\section{Mise en garde/Advice}

Afin de rendre accessible au plus Motivated by a desire to make the grand nombre le résultat des results of its graduate students' travaux de recherche menés par ses research accessible to all, and in étudiants gradués et dans l'esprit des accordance with the rules règles qui régissent le dépôt et la governing the acceptation and diffusion des mémoires et thèses diffusion of dissertations and produits dans cette Institution, theses in this Institution, the I'Université du Québec à Université du Québec à Chicoutimi (UQAC) est fière de Chicoutimi (UQAC) is proud to rendre accessible une version make a complete version of this complète et gratuite de cette œuvre. work available at no cost to the reader.

L'auteur conserve néanmoins la The author retains ownership of the propriété du droit d'auteur qui copyright of this dissertation or protège ce mémoire ou cette thèse. thesis. Neither the dissertation or Ni le mémoire ou la thèse ni des thesis, nor substantial extracts from extraits substantiels de ceux-ci ne it, may be printed or otherwise peuvent être imprimés ou autrement reproduced without the author's reproduits sans son autorisation. permission. 
À ma Femme

Denise

Et $\dot{a}$ mes enfants

Thomas, Simon et l'autre qui

s'en vient très bientôt. 


\section{Résumé}

Ce mémoire décrit sur une nouvelle méthode pour briser la glace en utilisant les ondes de choc. Le travail effectué a consisté à concevoir, fabriquer et caractériser un dispositif pouvant focaliser des ondes de choc dans l'air. Trois réflecteurs elliptiques ont été utilisés de façon à focaliser des ondes de choc sur un disque de glace. Les ondes de choc sont générées par une décharge électrique d'environ $10 \mathrm{kV}$ dans l'air à $-4,5{ }^{\circ} \mathrm{C}$ au foyer primaire du réflecteur. Les échantillons de glace qui ont un diamètre de $8,5 \mathrm{~cm}$ et une épaisseur variant de 3 à $10 \mathrm{~mm}$, sont placés au foyer secondaire. L'efficacité des réflecteurs a été caractérisée dans les directions axiales et radiales à l'aide d'un capteur de pression commercial. La pression maximale enregistrée au point focal est de 400 $\mathrm{kPa}$ (près de quatre fois la pression atmosphérique) avec un temps de montée de $2 \mu$ s. La rupture complète a été observée sur des échantillons d'une épaisseur maximale de 9,2 $\mathrm{mm}$ après un seul passage de l'onde de choc. Les échantillons dont l'épaisseur est comprise entre 9,2 et $10 \mathrm{~mm}$ se fracturent après plusieurs coups et finalement ceux dont l'épaisseur est supérieure à $10 \mathrm{~mm}$ ne se fracturent pas. Le processus de rupture semble être reliée à un processus de flexion en domaine fragile. Une hypothèse, reliée à la présence des bulles dans les échantillons de glace, a été proposée pour expliquer la rupture de ceux-ci par plusieurs coups successifs. 


\section{Remerciements}

À mon directeur de recherche, Jean-Louis Laforte, qui m'a donné l'occasion de faire une recherche originale et qui m'a supporté tout au long de ce projet.

À Gilles Lemire pour la réalisation des montages expérimentaux.

À tous les membres du L.I.M.A, pour qui la glace n'a (presque) plus de secrets.

À Claude D'Amours, un retraité qui vit sous haute tension.

Aux gars de l'électronique, Dany Ouellet, qui a toujours de l'équipement sous la main et Serge Gauthier, qui est plus qu'un simple fournisseur de fusible.

À Patrice Paquette, mon voisin de laboratoire pour son coffre d'outils

Au département de physique pour les petits morceaux manquants

À Daniel Bégin pour avoir fouillé dans sa réserve personnelle de pièces.

À Laslo Kish pour son oreille attentive et pour m'avoir initié au schlieren

À Martin Brouillette pour ses précieux conseils.

Je vous remercie tous. 


\section{Table des matières}

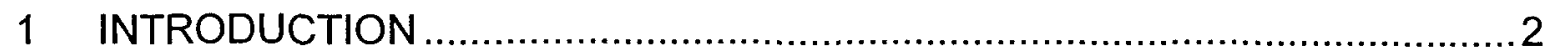

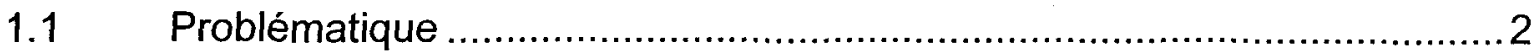

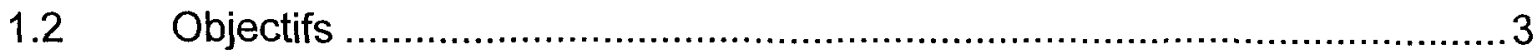

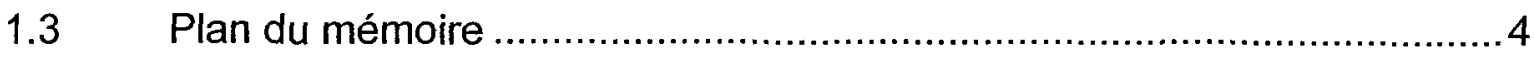

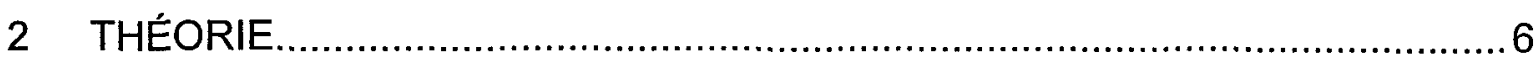

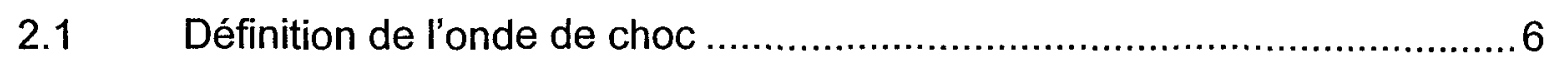

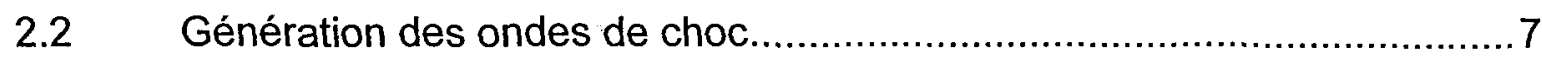

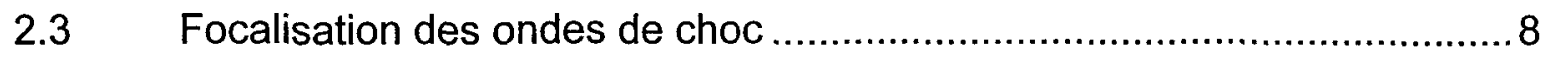

$2.4 \quad$ Interaction des ondes de choc avec les solides poreux ........................14

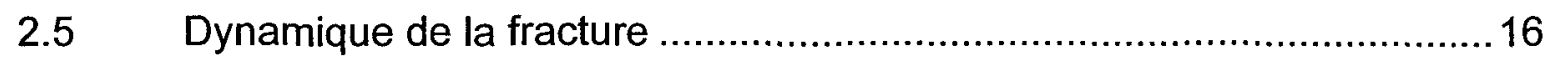

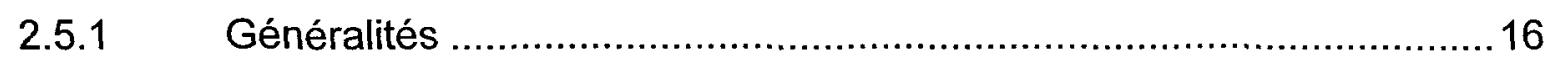

2.5.2 Processus de fracture de la glace sous ondes de choc focalisées ......20

2.5.3 Déflection d'une plaque mince .....................................................23

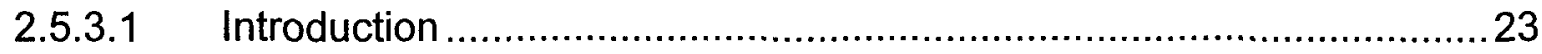

2.5.3.2 Méthode du théorème de l'énergie potentielle minimum.....................26

2.5.3.3 Calcul des contraintes dans les échantillons circulaires de glace ........33

3 MONTAGE EXPÉRIMENTAL ET ACQUISITION DES DONNÉES .................37

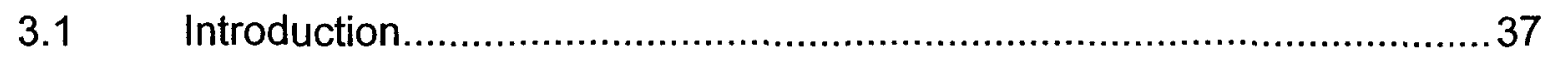

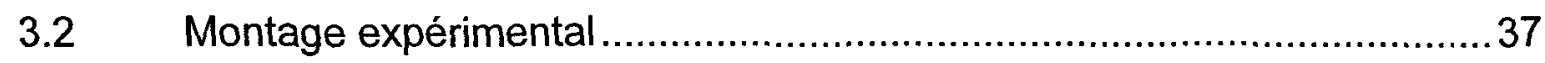

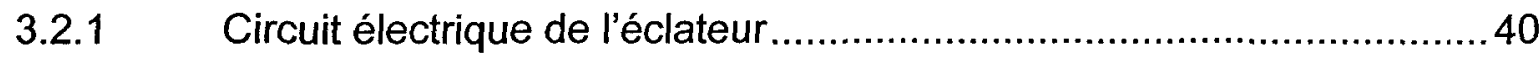

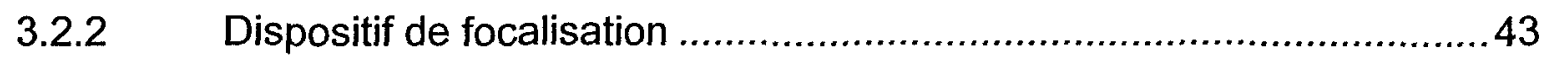

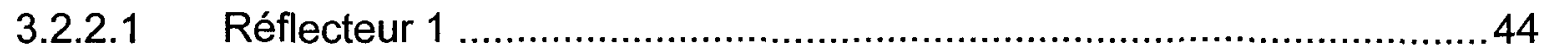




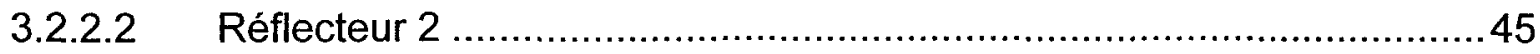

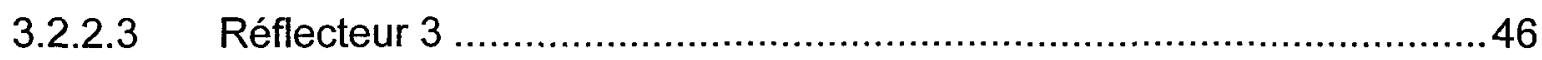

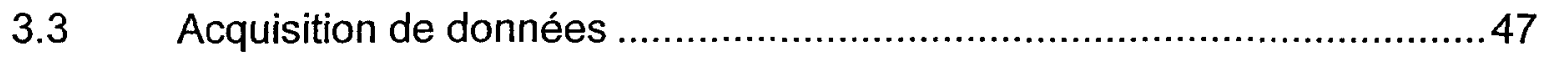

3.4 Montage pour le calcul de l'énergie de fracture ...................................48

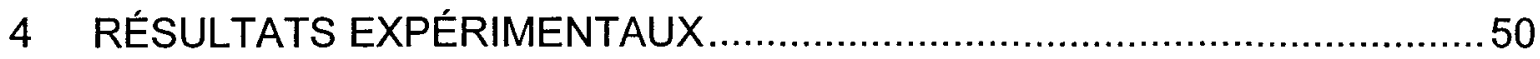

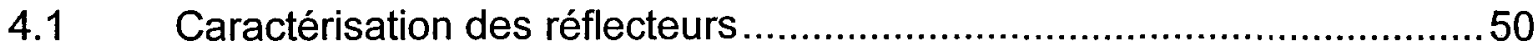

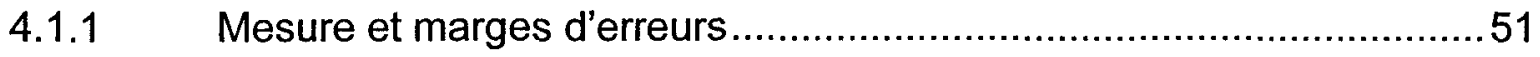

4.1.1.1 Erreur sur la mesure de la pression...............................................51

4.1.1.2 Erreur sur la position axiale et radiale..........................................51

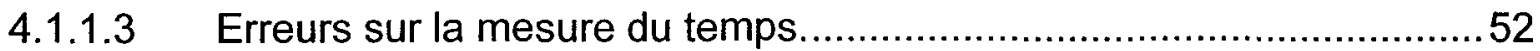

4.1.1.4 Erreur sur la dimension des échantillons ......................................52

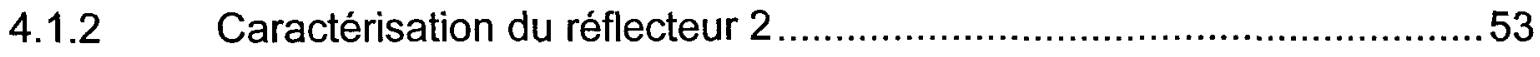

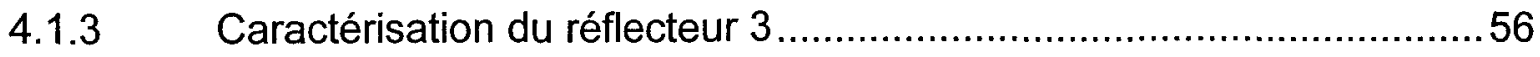

4.1.4 Sommaire de la caractérisation des réflecteurs ................................59

4.2 Caractéristiques des échantillons de glace ........................................60

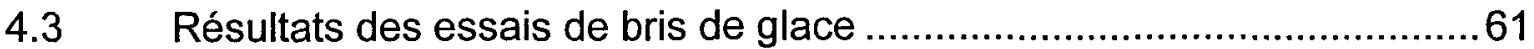

4.4 Résultats des mesures d'impact avec une bille ......................................68

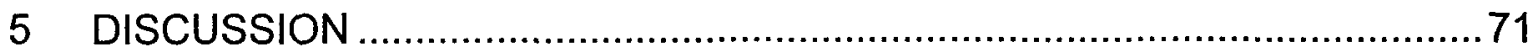

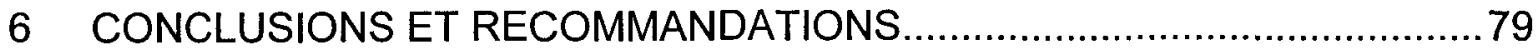

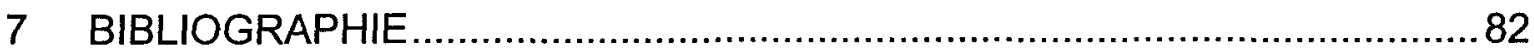




\section{Liste des Figures}

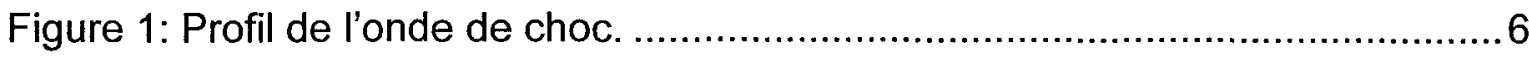

Figure 2: Réflexion d'une onde de choc sur une surface oblique. ....................... 10

Figure 3: Diffraction d'une onde de choc par un coin à $90^{\circ}$................................11

Figure 4 : Étapes successives de la focalisation par un réflecteur ellipsoïde.

L'onde se propage de gauche à droite................................................. 12

Figure 5: Réfraction d'une onde de choc sur une surface oblique.......................14

Figure 6 : Comportement d'un matériau ductile sous charge statique, ................17

Figure 7: Comportement d'un matériau à différentes vitesses de déformation ......17

Figure 8: Résistance en tension et en compression du verglas à $-2^{\circ} \mathrm{C}$ en fonction du taux de déformation. ................................................................... 19

Figure 9: Processus de fracture par onde de choc focalisée. .............................22

Figure 10: Plaque circulaire de rayon $b$ et d'épaisseur $h$..................................23

Figure 11: Contraintes radiales et tangentielles sur un élément de la plaque

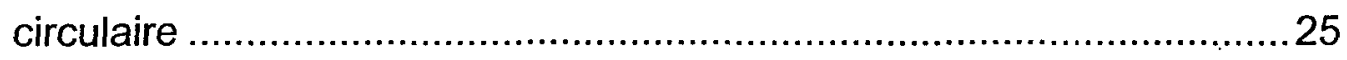

Figure 12: Plaque circulaire en flexion sous une distribution de charge

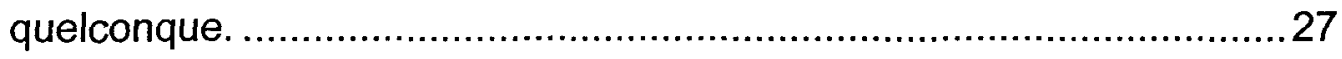

Figure 13: Déflection de l'échantillon en fonction du rayon.....................................34

Figure 14: Contraintes $\sigma_{\mathrm{rr}}$ et $\sigma_{\theta \theta}$ en fonction du rayon d'une plaque de $5 \mathrm{~mm}$

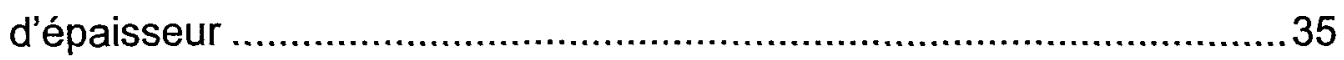

Figure 15: Montage utilisé pour la génération des ondes de choc..........................39 
Figure 16: Schéma du circuit de l'éclateur ......................................................... 41

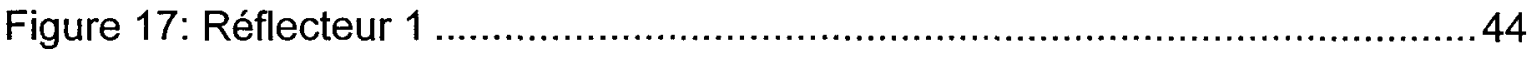

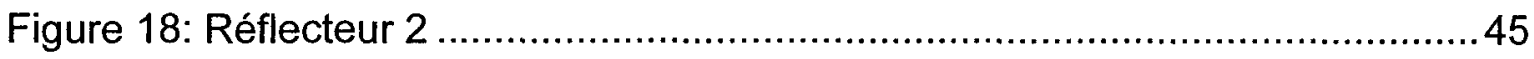

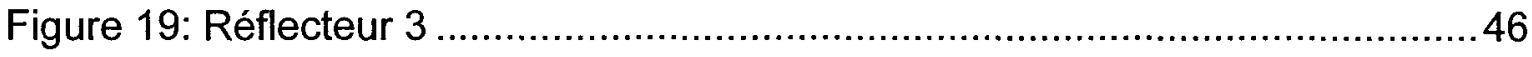

Figure 20: Montage utilisant une bille pour la briser les échantillons de glace. ....48

Figure 21: Pression en fonction de la distance axiale normalisée sur la distance

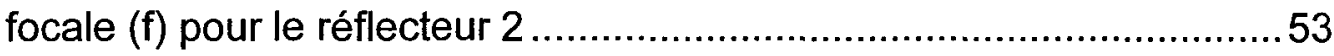

Figure 22 : Distribution radial de pression du réflecteur 2 évalué à $x / f=1,17 \ldots \ldots .54$

Figure 23: Vitesse du front de l'onde de choc pour le réflecteur 2 .......................55

Figure 24: Pression en fonction de la distance axiale normalisée sur la distance

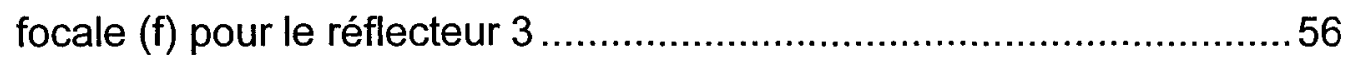

Figure 25: Distribution radiale de pression du réflecteur 2 évalué à $x / f=0,8 \ldots \ldots .57$

Figure 26: Vitesse du front de l'onde de choc pour le réflecteur $3 \ldots \ldots \ldots \ldots \ldots \ldots \ldots \ldots . . .58$

Figure 27: Fractures des échantillons de $3,7 \mathrm{~mm}, 5,2 \mathrm{~mm}$ et $6,2 \mathrm{~mm}$ avec le réflecteur 2 .

Figure 28: Fractures des échantillons de $3,1 \mathrm{~mm}, 4,4 \mathrm{~mm}$ et $5,9 \mathrm{~mm}$ avec le réflecteur 3 .

Figure 29: Échantillon de verglas d'une épaisseur 9,2 $\mathrm{mm}$ avec réflecteur 2 : aucun coup 64

Figure 30: Échantillon de verglas d'une épaisseur 9,2 $\mathrm{mm}$ avec réflecteur 2 : premier coup 64 
Figure 31 : Échantillon de verglas d'une épaisseur $9,2 \mathrm{~mm}$ avec réflecteur 2 : deuxième coup 65

Figure 32 Échantillon de verglas d'une épaisseur $9,2 \mathrm{~mm}$ avec réflecteur 2 : troisième coup 65

Figure 33: Échantillon de verglas d'une épaisseur 9,2 $\mathrm{mm}$ avec réflecteur 2 : quatrième coup 66

Figure 34: Échantillon de verglas d'une épaisseur $9,9 \mathrm{~mm}$ avec réflecteur 3 : aucun coup 67

Figure 35: Échantillon de verglas d'une épaisseur $9,9 \mathrm{~mm}$ avec réflecteur 3 : premier coup 67

Figure 36 : Résultats des essais de bris des échantillons de glace avec la bille. ..68

Figure 37: Comparaison entre le profil radial expérimental (a) et le profil théorique effectif (b). .72

Figure 38: Contraintes maximales théoriques au centre du disque en fonction de l'épaisseur. .74

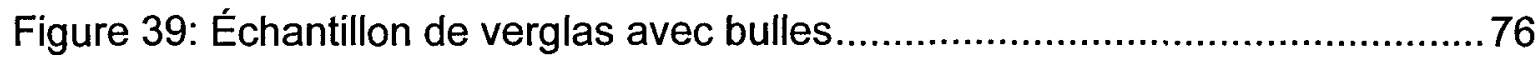

Figure 40: Mécanisme d'arrêt de la propagation de la fissure par une bulle..........77 


\section{Nomenclature}

b

D

E

$\mathrm{E}_{\mathrm{fr}}$

$\mathrm{f}$

g

$\mathrm{h}$

h

$\mathrm{m}$

$\mathrm{p}$

P.

$\mathrm{P}+$

$q(r)$

$\mathrm{T}$

Tm

$\mathrm{U}(\varepsilon)$

$\mathrm{V}$

$\mathrm{w}(\mathrm{r})$

$\dot{\varepsilon}$

$v$

$\rho$

$\sigma_{\text {fr }}$

$\sigma_{\mathrm{rr}}$

$\sigma_{z z}$

$\sigma_{\theta \theta}$
Rayon de l'échantillon

Module de rigidité

Module de Young

Energie nécéssaire a la fracture

Distance focale

Acceleration gravitationnelle $\left(9,8 \mathrm{~m} / \mathrm{s}^{2}\right)$

Épaisseur de l'echantillon

Hauteur

Masse

Poids

Raréfaction

Surpression

Distribution de charge transversale

Température

Temps de monte du pic de surpression

Energie interne totale de deformat

Vitesse

Déflection selon le rayon de l'échantillon

Vitesse de déformation

Coefficient de Poisson

Densité

Point de contrainte de rupture

Contraintes radiales

Contrainte perpendiculaire au plan

de l'échantillon

Contraintes tangentielles 
Chapitre 1

Introduction 


\section{Introduction}

\subsection{Problématique}

La glace atmosphérique cause de nombreux problèmes aux systèmes électriques et mécaniques. Ces systèmes peuvent être statiques (ponts, rails de chemin de fer, câbles électriques, etc.) ou dynamiques (bateaux, avions, éoliennes,etc.). C'est surtout dans les régions froides et tempérées de l'Amérique de nord, de l'Europe et de l'Asie que l'on retrouve ces problémes. Plusieurs travaux de recherches ont été effectués sur le sujet et une quarantaine de techniques ont été essayées pour déglacer plusieurs types de surfaces. Une liste exhaustive des techniques utilisées dans le domaine des transports et appliquées aux câbles aériens a été produite par Laforte et al. (1994). Les techniques de déglaçage sont réparties en deux grandes catégories soit : les techniques actives thermiques et mécaniques. Avec les techniques thermiques, la surface à déglacer est chauffée au-dessus du point de congélation alors qu'avec les techniques mécaniques, la glace est brisée en lui imposant des contraintes mécaniques qui excèdent sa résistance ou son adhérence : raclage, chocs, roulage, etc. Bien qu'habituellement plus complexes à mettre en œuvre, les techniques mécaniques requièrent un temps de déglaçage moindre et utilisent, en pratique, environ 100 fois moins d'énergie que les techniques thermiques pour provoquer le déglaçage, 
d'où l'intérêt de développer ce type de technique. Parmi celles-ci, les techniques par électro-expulsion qui déforme la surface de façon très rapide sont parmi les plus prometteuses, car elles produisent une fracture rapide de la glace en plusieurs petits morceaux, ce qui est essentiel dans plusieurs situations.

\section{$1.2 \quad$ Objectifs}

La recherche présentée dans ce mémoire propose une nouvelle approche mécanique, qui consiste essentiellement à utiliser les ondes de choc pour briser la glace en régime fragile. Cette façon de faire pourrait permettre de transmettre la déformation à la glace à partir de la surface extérieure de la glace et de briser la glace à distance sans devoir entrer en contact avec elle.

Pour vérifier la faisabilité de cette nouvelle approche, nous avons suivi les étapes suivantes :

- Conception et fabrication d'un générateur d'ondes de choc.

- Définition d'une procédure expérimentale.

- Conception et fabrication des échantillons.

- Utilisation d'un modèle mathématique de la fracture des échantillons.

- Proposition d'un modèle phénoménologique de la propagation des fissures. 


\subsection{Plan du mémoire}

Le chapitre 1 introduit la problématique et définit les objectifs du mémoire. Dans le chapitre 2, les aspects théoriques de l'étude sont présentés en trois parties. La première partie couvre la définition, la génération et la focalisation des ondes de choc. La deuxième partie décrit qualitativement l'interaction entre les ondes de choc et un solide, et la dernière partie aborde la dynamique de la fracture de la glace.

Le chapitre 3 est consacré à la description du montage expérimental qui comprend le générateur d'ondes de choc, les réflecteurs utilisés pour la focalisation et le système d'acquisition de donnés. Le chapitre 4 présente les résultats obtenus lors de la caractérisation des réflecteurs et des essais de rupture des échantillons de glace. Le chapitre 5 est consacré à la discussion des résultats où l'on propose une explication du processus de la rupture des échantillons de glace par coups successifs. Finalement, les conclusions et les recommandations apparaissent au chapitre 6 . 


\section{Chapitre 2}

Théorie 


\section{Théorie}

\subsection{Définition de l'onde de choc}

Une onde de choc se reconnaît facilement sur un graphique pression-temps à cause de sa forme en « $N »($ voir Figure 1). Elle est constituée d'une surpression supersonique $(P+)$, suivie d'une raréfaction subsonique (P-). En fait, comme la vitesse de chacun des points du profil de l'onde est différente, la forme du profil varie au cours du temps, ce qui explique la dispersion rapide de l'onde de choc. Le temps de montée en surpression (Tm) est de l'ordre d'une ou deux microsecondes dans l'air. Ces variations brusques de pression à l'intérieur du matériau sont susceptibles d'entraîner sa rupture en régime fragile. Le sujet de cette étude est donc d'étudier les effets de la transmission d'une onde de choc à l'interface airglace sur la rupture du verglas.

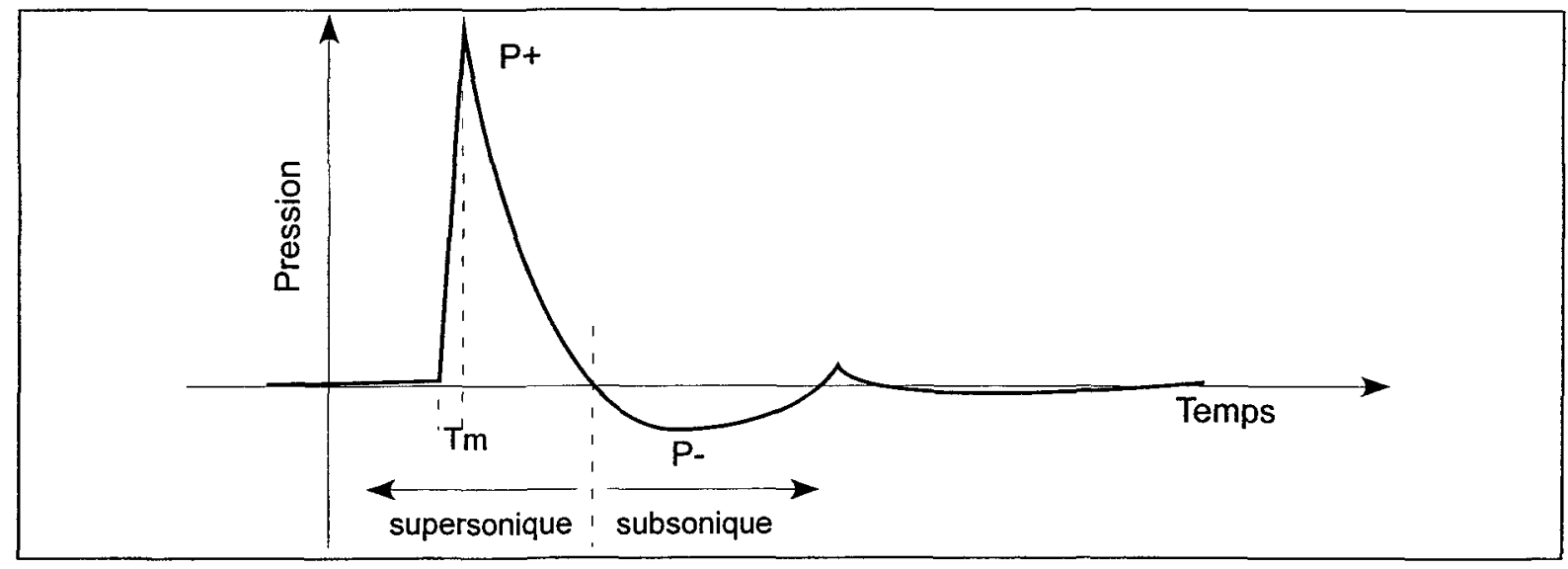

Figure 1: Profil de l'onde de choc. 


\subsection{Génération des ondes de choc}

De façon générale, une onde de choc est produite dans un milieu de deux façons différentes, soit : lorsqu'un objet rigide se déplace plus rapidement que la vitesse du son dans le milieu où il se trouve, ou encore lorsqu'il se produit une variation supersonique du volume du milieu comme dans le cas d'une explosion.

Plusieurs techniques sont utilisées en laboratoire pour produire des ondes de choc et le choix dépend surtout de l'état du milieu (solide, liquide ou gazeux) et de l'application visée.

Dans le cas d'un milieu solide la production d'ondes de choc se fait en utilisant des impacteurs, des explosifs et des impulsions laser en surface.

Dans un milieu liquide, les possibilités sont plus variées, on peut utiliser des dispositifs piézo-électriques, des alliages magnétostrictifs, des pistons électromagnétiques, des décharges électriques ou encore des explosifs. Par contre, dans les gaz, leur génération s'effectue surtout à l'aide de tubes à chocs. II serait fastidieux d'énumérer tous les types de tubes à chocs. Disons simplement qu'ils sont principalement constitués d'une chambre d'essai où la pression est faible et d'une chambre moteur où le gaz se trouve en surpression. Une mince pellicule métallique sépare les deux chambres et c'est la disparition rapide de cette pellicule qui permet l'écoulement supersonique du gaz moteur dans la chambre 
d'essai engendrant du même coup une onde de choc. La prise de mesure s'effectue dans la chambre d'essai.

La production en laboratoire d'ondes de choc à l'air libre est limité à l'utilisation d'explosif ou d'une décharge électrique brusque (le claquage électrique). Pour les fins de ce mémoire, les ondes de choc ont étés produites à partir du claquage électrique entre deux électrodes.

Lors d'une décharge électrique entre deux électrodes dans l'air libre, un arc est produit. L'apparition brusque de cet arc engendre une augmentation rapide de volume produisant une onde de pression sphérique. Le front de cette onde de pression se déplace à une vitesse supersonique, c'est donc une onde de choc. La hauteur du pic de pression de cette onde est fonction du voltage appliqué. Par contre la profondeur de la partie en raréfaction est limitée par la pression atmosphérique. En décharge libre, le voltage de décharge est principalement fonction de l'écartement des électrodes, du niveau d'ionisation de l'air et dans une moindre mesure de l'humidité, la pression, la température et la teneur en aérosol.

\subsection{Focalisation des ondes de choc}

La focalisation des ondes de choc par un réflecteur ellipsoïde est principalement utilisée en médecine pour fractionner les calculs rénaux qui seront 
ensuite évacués par les voies naturelles. Les appareils utilisés sont appelés lithotriteurs électrohydrauliques et fonctionnent en milieu liquide. L'onde de choc est produite par une décharge électrique au foyer primaire du réflecteur ellipsoïde et l'appareil est disposé de façon à ce que la pierre soit située au foyer secondaire de l'ellipse. Pour ce type d'appareil, l'intensité des ondes de choc au foyer secondaire varie entre 30 et $40 \mathrm{MPa}$ (Cleveland et al., 2000).

L'onde de choc peut-être considérée comme une discontinuité dont l'épaisseur est négligeable, cette particularité permet de développer un modèle de propagation géométrique relativement simple à partir des fronts d'ondes.

Le phénomène le plus important qui affecte la propagation non-linéaire des fronts d'onde est l'apparition des ondes de Mach. Ce phénomène apparaît lors de la réflexion, de la réfraction et la focalisation des ondes de choc. L'exemple classique est la réflexion d'une onde de choc sur une surface oblique (voir figure 2).

Si l'onde de choc est de faible intensité, l'onde réfléchie suit les mêmes lois que les ondes optiques et acoustiques où l'angle incident est égal à l'angle réfléchi. La réflexion est dite régulière. (voir figure 2a) 
Par contre, pour une onde de choc forte ou pour une pente qui augmente graduellement, l'onde réfléchie rejoint l'onde incidente et il y a formation d'une onde de Mach (voir figure $2 \mathrm{~b}$ ), cette réflexion est dite irrégulière.

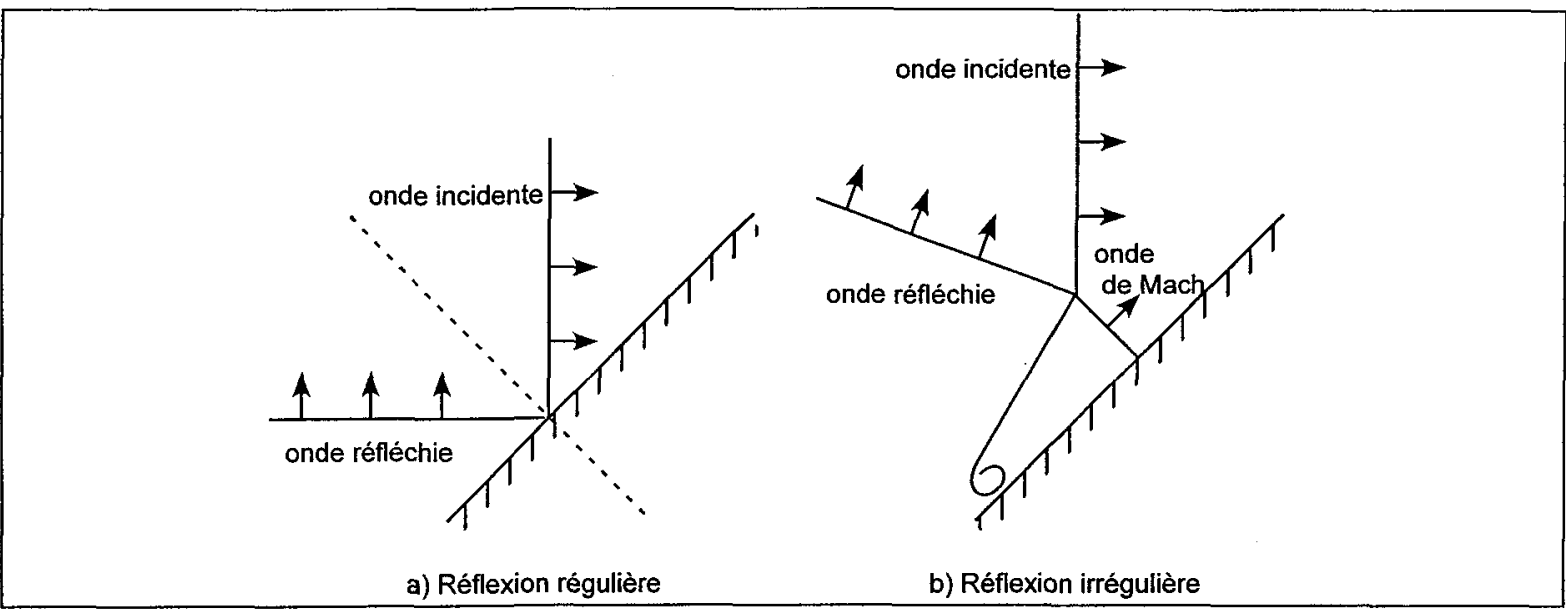

Figure 2: Réflexion d'une onde de choc sur une surface oblique.

La réflexion irrégulière a pour effet de produire trois ondes de compression d'intensités et d'orientations différentes. De plus, comme l'onde de Mach accélère le fluide à une vitesse supérieure aux deux autres, il y a formation d'un tourbillon qui tourne dans le sens anti-horaire.

Dans un réflecteur ellipsoïde, l'onde de choc se propage sphériquement à partir du point focal vers les parois du réflecteur. Près de la sortie du réflecteur, étant donné l'angle d'incidence, il y a formation d'une onde de Mach. La figure 3 illustre l'approximation acoustique de la diffraction d'une onde de choc autour d'un coin à $90^{\circ}$. 


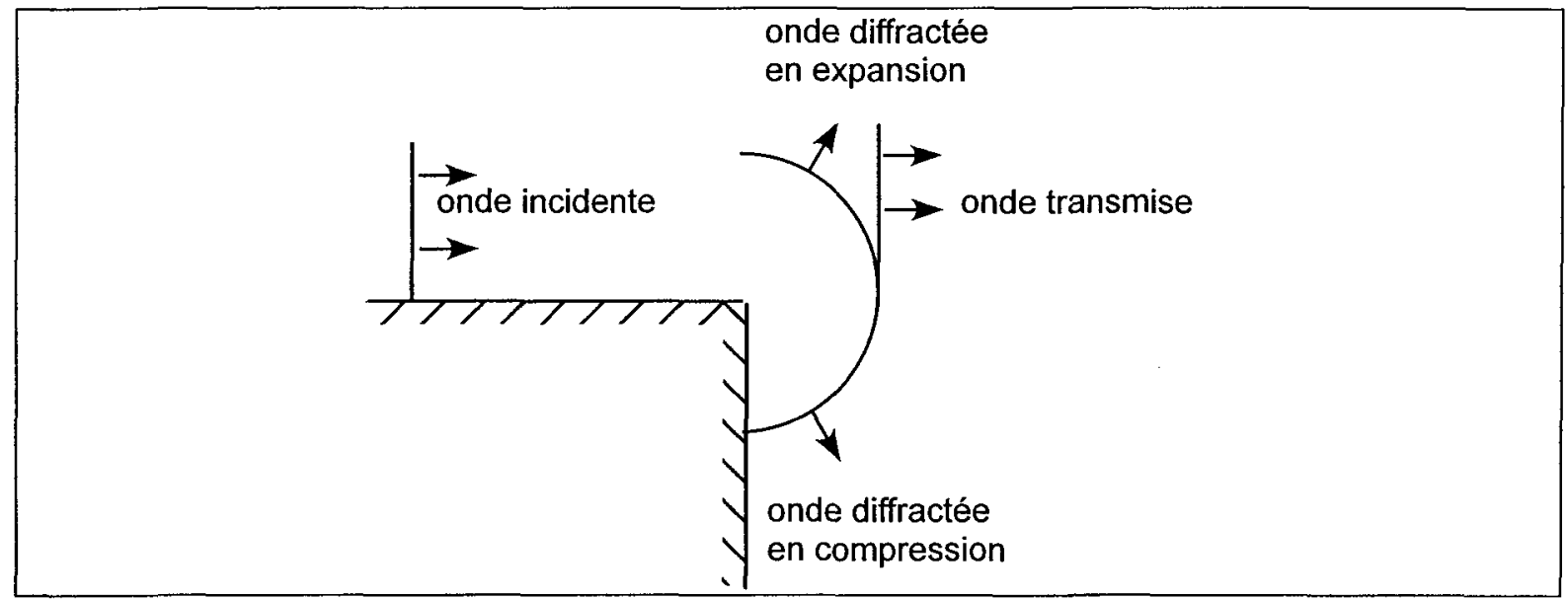

Figure 3: Diffraction d'une onde de choc par un coin à $90^{\circ}$.

L'onde de Mach à la sortie du réflecteur se comporte à peu près de la même façon. Par contre, étant donné que la réflexion est irrégulière, elle est détachée du mur et rejoint l'axe de l'ellipse. Sturtevant (2000), décrit bien le processus de focalisation de l'onde de choc par un réflecteur ellipsoïde (voir figure 4) 


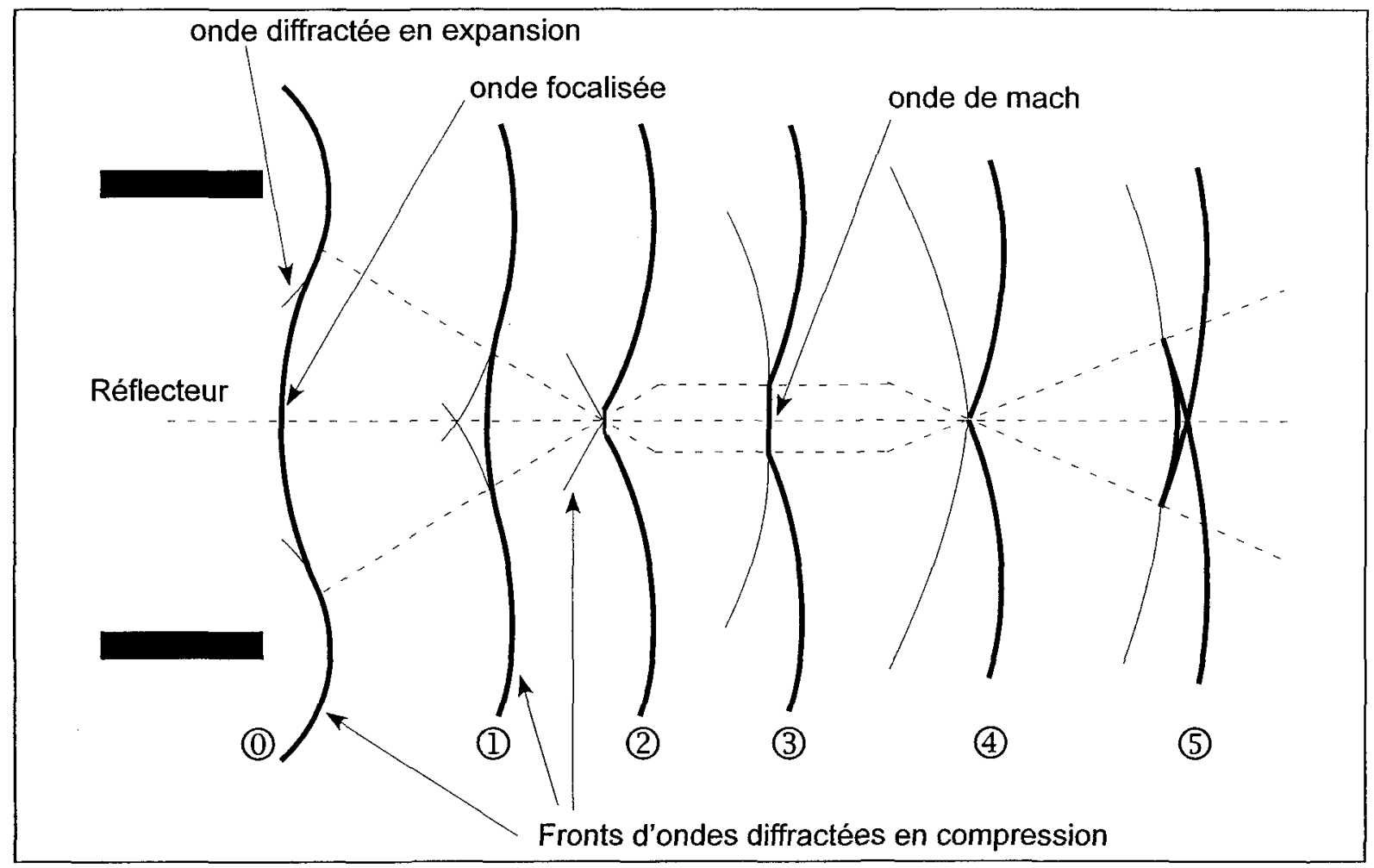

Figure 4: Étapes successives de la focalisation par un réflecteur ellipsoïde. L'onde se propage de gauche à droite

Dans la figure 4, on peut voir, évoluant dans le temps, le processus réel de focalisation d'une onde de choc, la progression de l'onde de choc se faisant de gauche à droite est illustrée en six étapes, numérotées de 0 à 5 . Tout d'abord, on remarque que contrairement à la théorie linéaire acoustique, l'onde de choc ne se focalise pas en un point, car la pression augmenterait à une valeur infinie à ce point. L'interaction choc-choc avant le point focal entraîne l'apparition d'une onde de Mach. Ce phénomène non-linéaire, mis en évidence par Schwendeman et 
Whitham (1987), prend naissance entre l'étape 1 et 2 . La partie interne de l'onde de choc diffractée en expansion devient près de l'axe, une onde de compression. Ce phénomène est semblable à la réflexion irrégulière d'une onde de choc (voir figure 2) où l'onde focalisée devient l'onde de Mach et l'onde diffractée devient l'onde réfléchie. Entre l'étape 2 et 4 se trouve la zone de focalisation où l'onde de Mach produit une compression d'intensité maximale.

Le fait que notre dispositif de focalisation soit utilisé dans l'air plutôt que dans un liquide constitue la différence majeure avec le lithotriteur électrohydraulique. II faut donc s'attendre à des pressions moindres et au réchauffement de la zone de décharge électrique au niveau des électrodes. Cette dernière particularité a pour conséquence que l'onde réfléchie par la paroi en arrière des électrodes se trouve à passer au travers d'une région réchauffée par l'explosion avant de rejoindre la région de focalisation. Cette partie de l'onde de choc sera donc réfractée par le changement de milieu et sa vitesse augmentera par rapport à l'autre partie réfléchie par les parois latérales. Les conséquences exactes sur la zone focale sont difficiles à quantifier. Néanmoins, en se basant sur les interférogrammes faits par Schelin, Gren et Wâblin (1997) avec une cavité ellipsoïde fermée, on peut prévoir un déplacement de la zone de focalisation, ce qui ne change rien à l'essentiel du phénomène. 


\section{$2.4 \quad$ Interaction des ondes de choc avec les solides poreux}

Lorsqu'une onde de choc frappe un solide rigide, une partie de l'onde est réfléchie alors que l'autre partie est réfractée (transmise). Comme pour la partie réfléchie l'onde réfractée peut être régulière ou irrégulière.

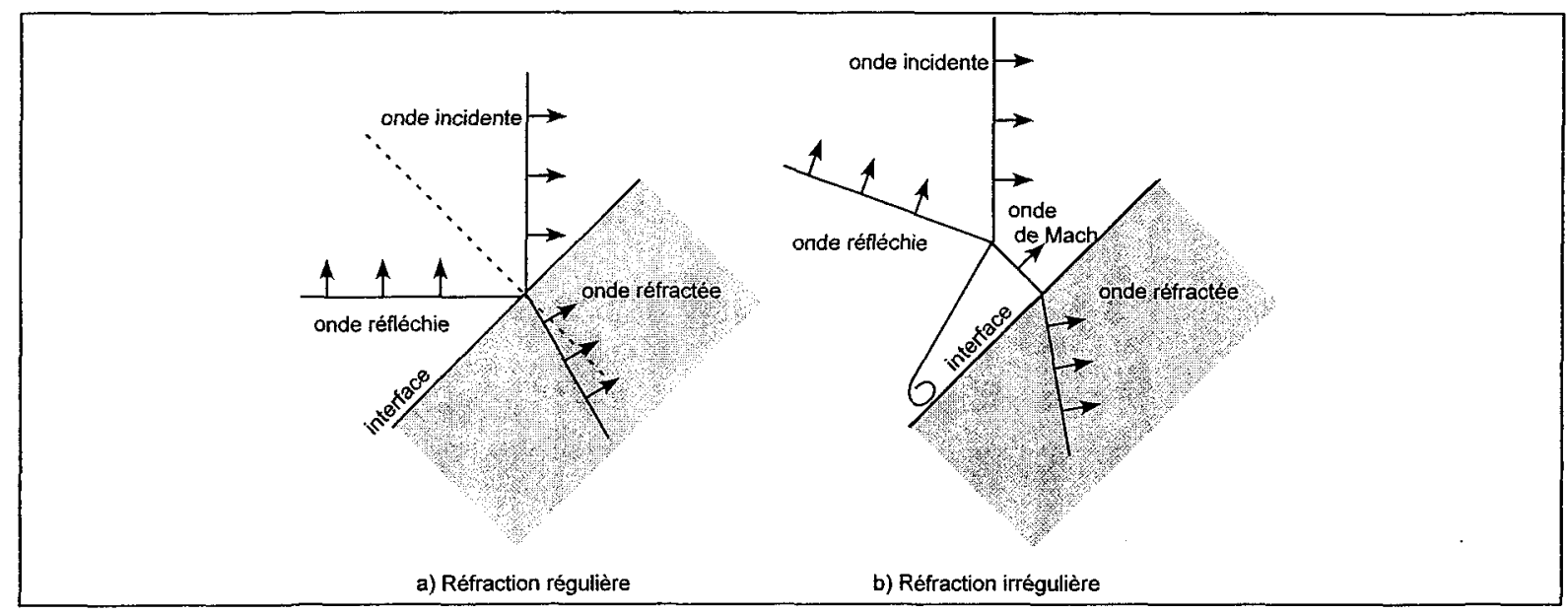

Figure 5: Réfraction d'une onde de choc sur une surface oblique.

La partie réfractée de l'onde de choc dans un solide comprend trois types d'ondes : des ondes de compression, de cisaillement et des ondes de surface par " conversion de mode". Les ondes de compression sont les plus intenses et contribuent davantage à la rupture du matériau. La transmission de ces ondes dépend des propriétés du matériau. Pour la glace poreuse, l'onde transmise entre en collision avec les bulles d'air et subit une succession de réfractions et de réflexions causées par la variation d'impédance. 
Ainsi, une partie de l'onde entraînera une pression dans le matériau, une autre traversera celui-ci et finalement la dernière sera diffusée par les bulles. Ce phénomène est complexe et dépend de plusieurs facteurs : la rigidité, la perméabilité et densité relative du matériau, les dimensions des bulles, la variation d'impédance à l'interface et l'angle d'entrée de l'onde incidente. De nombreuses études ont été publiées sur le sujet notons entre autres Lokhandwalla et Sturtevant (2000) pour les calculs rénaux, Kitagawa et al. (2001) pour l'étude des coefficients d'entraînements pour des mousses synthétiques flexibles ou encore Levy et al. (1993) pour des corps rigides et poreux. Aucune étude n'a été publiée sur la glace poreuse à cellule fermée. De plus, les études sur le sujet sont surtout de nature qualitative et ne permettent pas de modéliser avec exactitude la pression effectivement captée par le matériau dans le cas de la glace. 


\subsection{Dynamique de la fracture}

\subsubsection{Généralités}

Le comportement mécanique des matériaux sollicités mécaniquement par une force extérieure est habituellement décrit par la courbe contrainte-déformation qui est obtenue à partir des essais de traction en laboratoire. La Figure 6, illustre le comportement d'un matériau ductile. La courbe se divise en deux zones principales. La première, la zone élastique, est caractérisée par un comportement qui suit la loi de Hooke, où la déformation est linéaire et réversible. La deuxième zone, montre un comportement plastique non-linéaire, où la déformation est irréversible, qui se termine par la rupture du matériau. L'aire sous la courbe est proportionnelle à l'énergie nécessaire pour atteindre le point de rupture $\left(\sigma_{\mathrm{fr}}\right)$. La température, les défauts, la structure cristalline et la vitesse de chargement sont des facteurs qui peuvent modifier l'allure de cette courbe de comportement. 


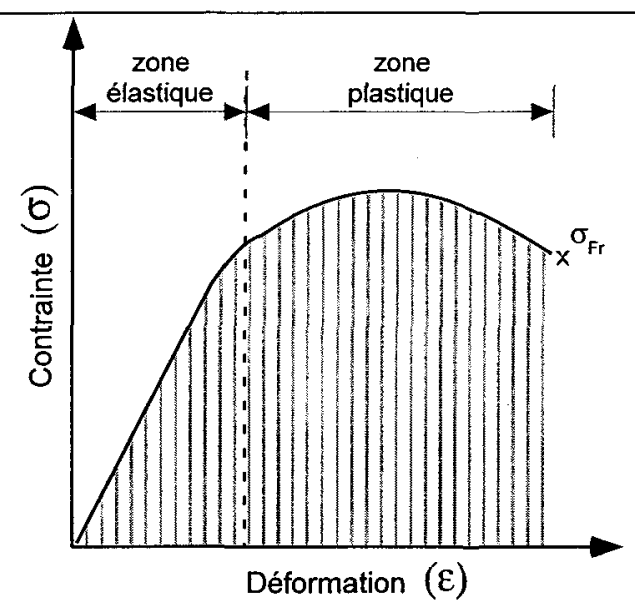

Figure 6 : Comportement d'un matériau ductile sous charge statique,

La Figure 7, illustre l'effet de la vitesse de déformation $(\quad \dot{\varepsilon})$ sur le comportement mécanique d'un matériau. Si la vitesse de chargement est augmentée, la zone plastique diminue, la contrainte de rupture $\left(\sigma_{\mathrm{fr}}\right)$ prend une valeur plus élevée et le matériau devient plus fragile. Par contre, l'énergie requise pour atteindre le point de rupture est moindre, car elle n'est pas dissipée par la déformation plastique.

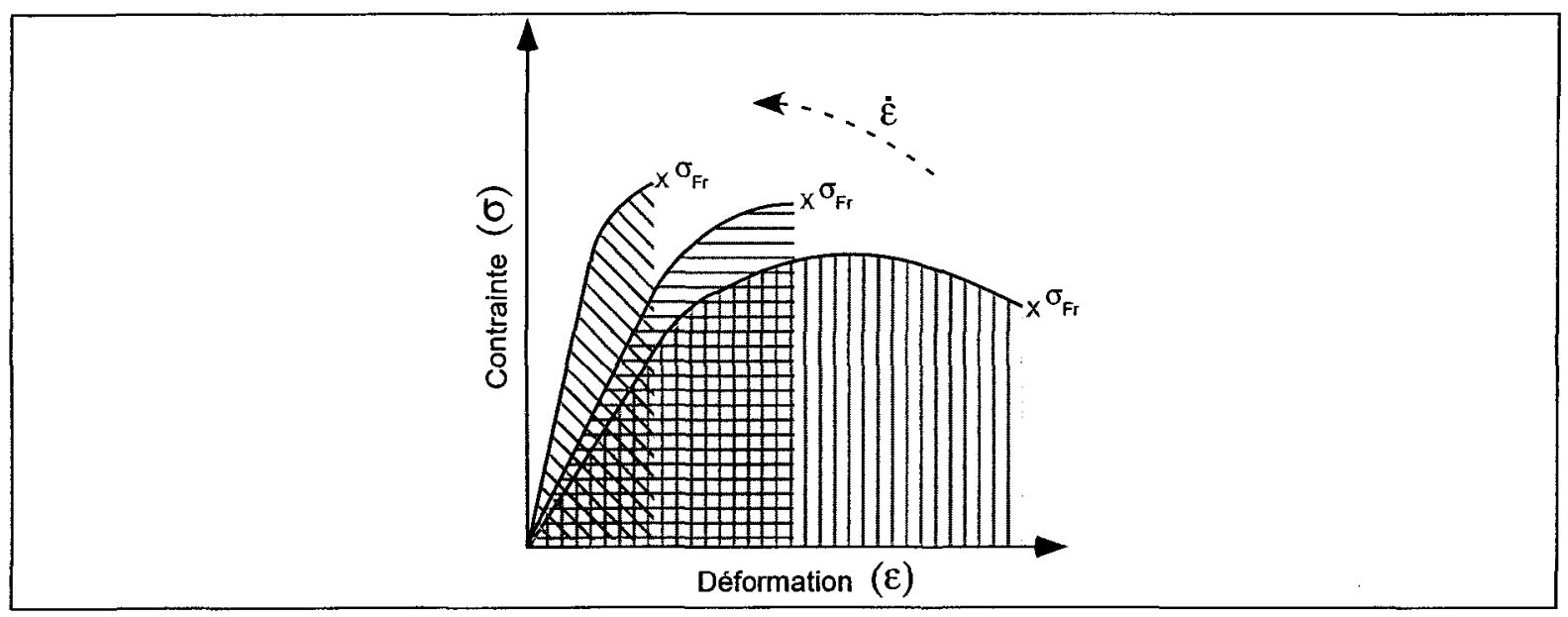

Figure 7: Comportement d'un matériau à différentes vitesses de déformation 
Les contraintes de ruptures en tension et en compression ont été mesurées par Laforte et Nguyen (1991) sur des échantillons de verglas à $-2^{\circ} \mathrm{C}$ pour différentes vitesses de chargement. La figure 8 illustre les résultats obtenus. Le graphique est divisé en trois régions qui correspondent chacune à un intervalle défini de déformation. Dans la première région $\left(\dot{\varepsilon}<10^{-5} / \mathrm{s}\right)$, le verglas se déforme lentement de façon ductile et le point de rupture est identique en tension et en compression. Dans la deuxième région $\left(10^{-5} / \mathrm{s}<\dot{\varepsilon}<10^{-3} / \mathrm{s}\right)$, le verglas est ductile en compression mais fragile en tension. Finalement, dans la troisième région ( $\dot{\varepsilon}>10^{-3} / \mathrm{s}$ ), le verglas se déforme de façon très rapide et devient fragile en compression et en tension. Par contre, la contrainte de rupture en compression est une fois et demie plus élevée que la contrainte de rupture en tension. Cet écart augmente lorsque la température de la glace atmosphérique diminue (voir tableau 2). Ainsi, pour une même densité $\left(900 \mathrm{~kg} / \mathrm{m}^{3}\right)$, l'écart entre la contrainte de rupture en compression et en tension passe de $0,6 \mathrm{~N} / \mathrm{m}^{2}$ à $8,4 \mathrm{~N} / \mathrm{m}^{2}$ entre $-2{ }^{\circ} \mathrm{C}$ et $-14^{\circ} \mathrm{C}$

La formation de la glace implique le développement de plusieurs lieux de concentration de contraintes, micro-fissures et bulles, qui peuvent entraîner la formation d'une fissure qui, sous certaines conditions, entraînera la rupture de l'échantillon. Par contre, il est expérimentalement démontré (Gold,1960; Michel et al.,1978) que la glace se brise à des seuils de tension inférieurs à ceux qui sont 
engendrés par ces défauts. En fait, la condition critique de formation d'une fissure est le relâchement de l'énergie élastique d'un grain de glace individuel (Michel,1978). Dans une rupture fragile en tension, cette fissure se propage de façon catastrophique au travers de l'échantillon.

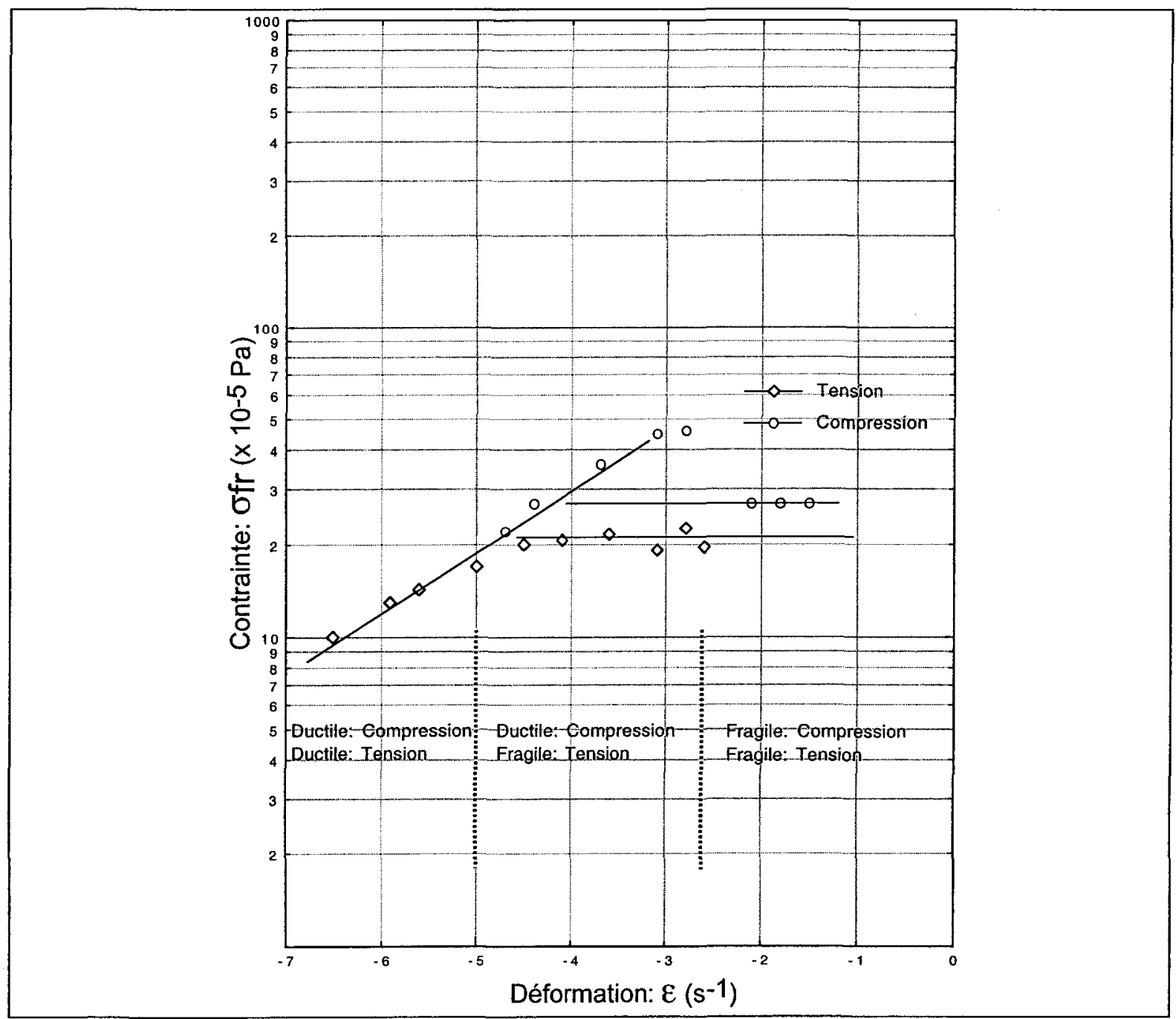

Figure 8: Résistance en tension et en compression du verglas à $-2^{\circ} \mathrm{C}$ en fonction du taux de déformation. 
Tableau 2 : Caractéristiques mécaniques des dépôts de verglas et de givre dur à la rupture fragile

\begin{tabular}{|c|c|c|c|c|}
\hline Type & $\begin{array}{c}\text { Densité } \\
\rho\end{array}$ & $\begin{array}{c}\text { Module de } \\
\text { Young } \\
\mathrm{E}\end{array}$ & $\begin{array}{c}\text { Contrainte de } \\
\text { rupture }\left(\sigma_{\mathrm{fr}}\right) . \\
\text { (Compression) }\end{array}$ & $\begin{array}{c}\text { Contrainte de } \\
\text { rupture }\left(\sigma_{\mathrm{fr}}\right) . \\
\text { (Tension) }\end{array}$ \\
\hline & $\mathrm{Kg} / \mathrm{m}^{3}$ & $\mathrm{~N} / \mathrm{m}^{2}$ & $\mathrm{~N} / \mathrm{m}^{2}$ & $\mathrm{~N} / \mathrm{m}^{2}$ \\
\hline Verglas $\left(-2^{\circ} \mathrm{C}\right)$ & 900 & $1 \times 10^{10}$ & $2,7 \times 10^{6}$ & $2,1 \times 10^{6}$ \\
\hline Givre $\left(-14^{\circ} \mathrm{C}\right)$ & 900 & $1 \times 10^{10}$ & $11 \times 10^{6}$ & $2,6 \times 10^{6}$ \\
\hline
\end{tabular}

\subsubsection{Processus de fracture de la glace sous ondes de choc focalisées}

Lorsqu'une onde de choc est focalisée sur un disque mince de glace, deux phénomènes peuvent se produire. Le premier est une flexion du disque causée par la distribution de pression. Ce phénomène engendrera des réactions internes de tension et de compression. Celles-ci seront expliquées dans la section 2.5.3. Le deuxième phénomène est associé directement au passage de l'onde de choc à l'intérieur du matériau. Une pointe de pression suivie d'une pointe de tension traversera tout le matériau et les défauts (les bulles ou les micro-fissures) serviront de point de réflexion et d'amplification. Ce processus peut engendrer, si les contraintes sont suffisamment importantes, la décohésion du matériau par 
croissance des micro-fissures dans le domaine plastique. Sturtevant (2001) a utilisé une approche basée sur ce type d'interaction pour expliquer la fracture des calculs rénaux après plusieurs coups successifs, appliqués à l'aide d'ondes de choc. En lithotritie, les ondes de choc sont transmises à l'interface eau-calculs rénaux et atteignent des intensités de $40 \mathrm{MPa}$ en compression et de $10 \mathrm{MPa}$ en tension pour une contrainte de rupture en tension des calculs rénaux d'environ 1 $\mathrm{MPa}$. En ce qui concerne l'expérience menée dans ce mémoire, l'interface airglace est moins efficace pour produire et transmettre les ondes de choc. L'intensité moyenne des pointes de pression est de $0,350 \mathrm{MPa}$ (environ 3,5 fois la pression atmosphérique) et celles de tension sont limitées par la pression atmosphérique, pour des échantillons de glace dont la contrainte de rupture en tension est de 2,1 MPa. II est donc évident que ce phénomène de décohésion engendré par le passage de l'onde de choc dans le matériau, ne peut jouer aucun rôle dans la fracture des échantillons et que les contraintes locales dans le matériau sont dues principalement aux efforts de tension engendrés par le processus de flexion (voir figure 9). 


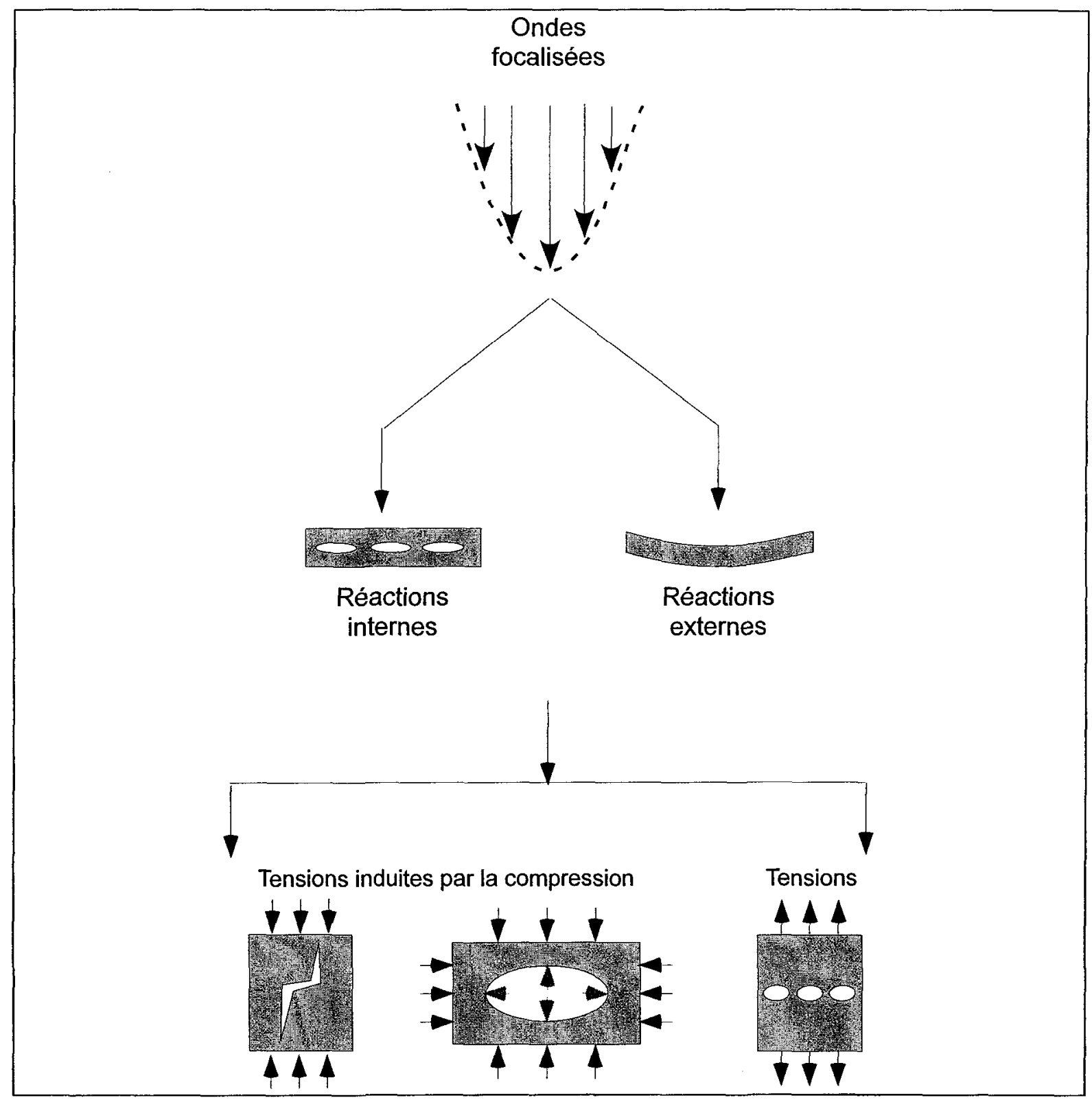

Figure 9: Processus de fracture par onde de choc focalisée. 


\subsubsection{Déflection d'une plaque mince}

\subsubsection{Introduction}

Une distribution de charge qui agit, perpendiculaire au plan, sur plaque mince produit une déflexion. Le calcul de cette déflection permet d'établir les contraintes à l'intérieur de la plaque. Les calculs qui suivent vont nous permettre d'établir deux relations, la première est la contrainte interne en fonction du rayon et la deuxième est la contrainte maximale en fonction de l'épaisseur de la plaque circulaire.

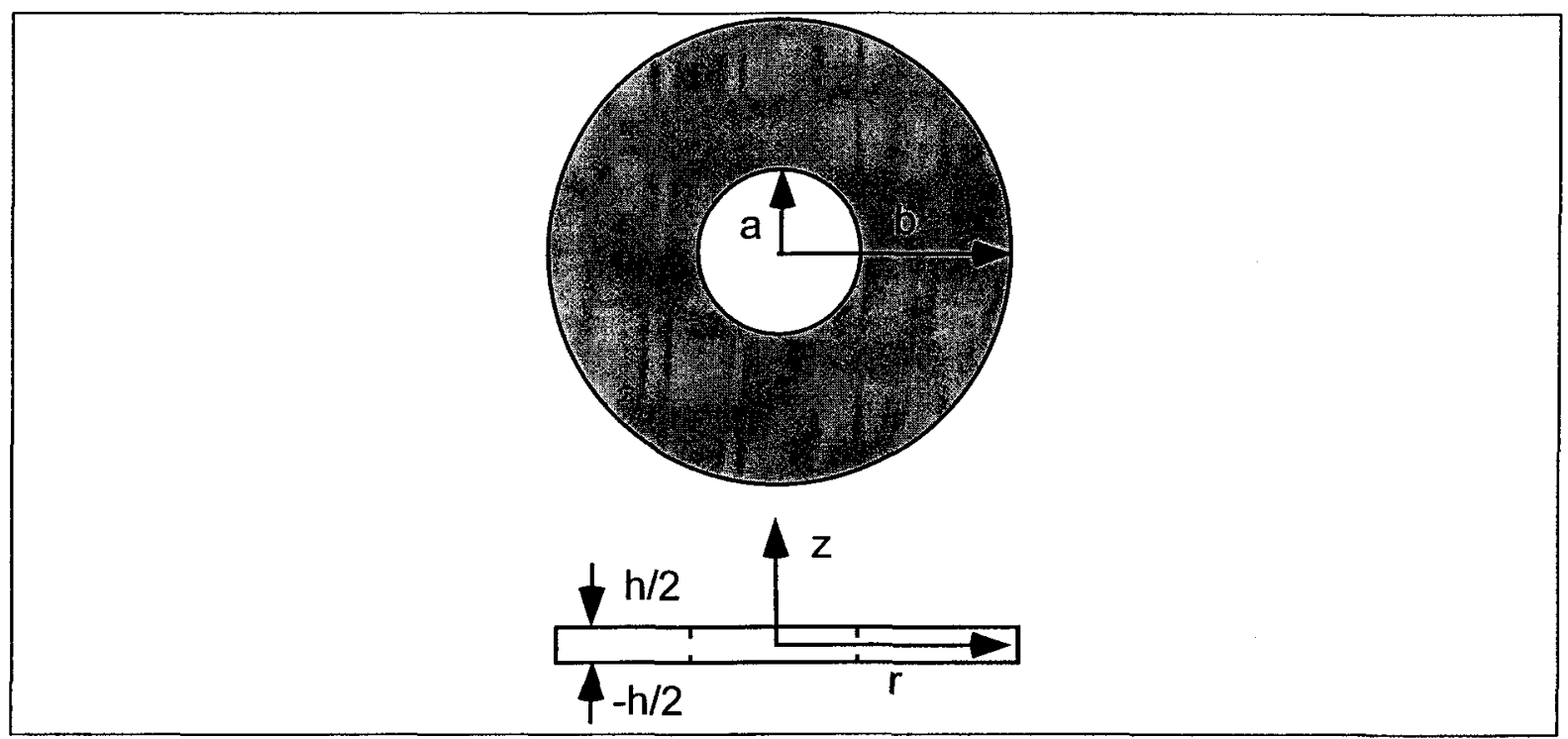

Figure 10: Plaque circulaire de rayon $b$ et d'épaisseur $h$ 
Soit un disque de rayon « $b$ » et d'épaisseur « $h$ », le développement du modèle est basé sur les hypothèses suivantes :

- L'épaisseur est petite par rapport au diamètre $(h<<)$

- La contrainte perpendiculaire au plan est négligée $\left(\sigma_{z z}=0\right)$

- La charge est appliquée perpendiculairement

- Aucune déformation en cisaillement

- Le matériau est homogène et isotrope

Le système de référence du disque est $0 \leq \theta \leq 2 \pi,-h / 2 \leq z \leq h / 2$ et $a \leq r \leq b$. Pour évaluer la déflection au centre du disque, la frontière « a » du rayon sera évaluée à la limite où a tend vers zéro. La déflection $w(r)$ sera positive dans le sens positif de l'axe z. Dans ces conditions le déplacement d'une point matériel de la plaque circulaire est donné par :

$u_{r}(r, z)=-w^{\prime}(r) z, \quad u_{\theta}(r, z)=0, \quad u_{z}(r, z)=w(r)$

Les contraintes correspondantes sont :

$$
\varepsilon_{r r}(r, z)=\frac{\partial u_{r}}{\partial r}=-\mathrm{w}^{\prime \prime}(\mathrm{r}) \mathrm{z} \quad \varepsilon_{\theta \theta}(r, z)=\frac{u_{r}}{r}=-\mathrm{w}^{\prime}(\mathrm{r}) \frac{\mathrm{z}}{\mathrm{r}}
$$


En considérant que $\sigma_{z z}=0$, la loi de Hooke prend la forme :

$\varepsilon_{r r}=\frac{1}{E} \sigma_{r r}-\frac{v}{E} \sigma_{\theta \theta}, \quad \varepsilon_{\theta \theta}=\frac{1}{E} \sigma_{\theta \theta}-\frac{v}{E} \sigma_{r r}$

Que l'on peut réécrire :

$\sigma_{r r}=\frac{E}{1-v^{2}}\left(\varepsilon_{r r}-v \varepsilon_{\theta \theta}\right), \quad \sigma_{\theta \theta}=\frac{E}{1-v^{2}}\left(\varepsilon_{\theta \theta}-v \varepsilon_{r}\right)$

où $E$ est le module de Young du matériau et $v$ est le coefficient de Poisson. Les contraintes $\sigma_{\pi r}$ et $\sigma_{\theta \theta}$ sont respectivement les contraintes radiales et tangentielles dans la plaque circulaire (voir figure 11).

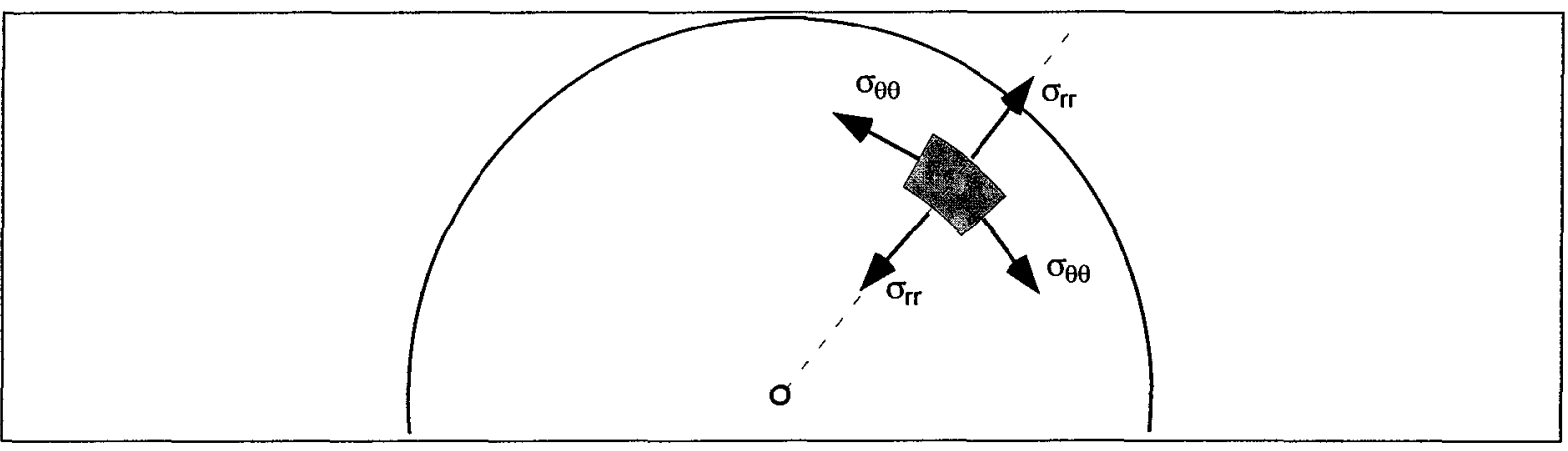

Figure 11: Contraintes radiales et tangentielles sur un élément de la plaque circulaire 


\subsubsection{Méthode du théorème de l'énergie potentielle minimum}

Dans les systèmes mécaniques, l'énergie est définie comme la capacité de faire un travail, et le travail est défini comme le produit de la force par la distance dans la direction du déplacement de la force. Dans les solides déformables, les forces sont les contraintes multipliées par leurs surfaces et les déformations représentent les distances. Le produit de ces deux quantités représente le travail interne fait par les forces externes appliquées sur le corps. Ce travail interne est emmagasiné dans le corps sous forme d'énergie élastique de déformation interne.

La mécanique d'une plaque élastique peut-être décrite par la méthode du théorème de l'énergie minimum. En tenant compte des conditions frontières, cette approche permet d'établir les équations différentielles nécessaires au calcul des déflections transversales $(w(r))$ à partir de leurs variations. Par la suite, la valeur de ces déflections permet de calculer les contraintes internes dans la plaque circulaire.

On considère une plaque circulaire pleine $(\mathrm{a}=0)$ qui est simplement supportée par son rebord $(w(b)=0)$ qui est soumise à une distribution de charge transversale $q(r)$ agissant dans le sens des $z$ négatif (vers le bas). La distribution de charge est quelconque selon le rayon, mais constante selon l'angle. La surface supérieure $(z=h / 2)$ subira des contraintes en compression, tandis que la surface 
inférieure $(z=-h / 2)$ subira des contraintes en tension (voir Figure 12). Étant donné la symétrie de la plaque qui est homogène et isotrope, l'axe neutre est situé au centre $(z=0)$.

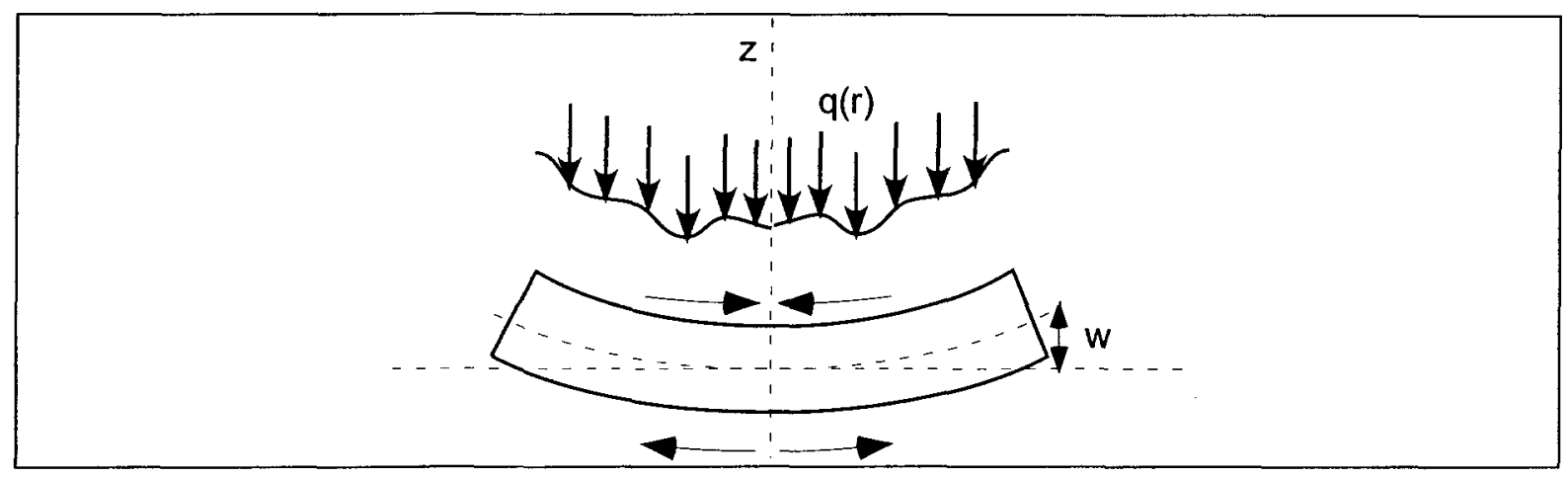

Figure 12: Plaque circulaire en flexion sous une distribution de charge quelconque.

Les dimensions physiques de $q(r)$ sont: $N / m^{2}$. L'énergie potentielle externe total est :

$$
-\int_{0}^{2} \int_{0}^{2 \pi} q(r) m(r) r d \theta d r=-2 \pi \int_{0}^{\pi} q(r) m(r) r d r
$$

L'énergie interne totale de déformation est obtenue en faisant la somme des densités d'énergie emmagasinée sous forme élastique dans le corps sur le volume de celui-ci. 
Dans notre cas, la loi de Hooke (équation 4) permet d'exprimer la densité d'énergie élastique sous la forme :

$$
U(\varepsilon)=\frac{1}{2} \sigma_{r} \varepsilon_{r}+\frac{1}{2} \sigma_{\theta \theta} \varepsilon_{\theta \theta}+\frac{1}{2} \sigma_{z z} \varepsilon_{z z}=\frac{E}{1-v^{2}}\left(\varepsilon^{2}{ }_{r}+\varepsilon_{\theta \theta}^{2}+2 v \varepsilon_{\pi} \varepsilon_{\theta \theta}\right)
$$

Pour obtenir l'énergie interne totale de déformation, on intègre $U(\varepsilon)$ sur le volume du disque en tenant compte des définitions présentées aux équations 4,5 et 6

$$
\int_{0}^{2} \int_{0}^{2} \int_{-h / 2}^{h} U(\varepsilon) d z r d \theta d r=\pi D \int_{0}^{2}\left(r w^{\prime \prime}(r)^{2}+\frac{w^{\prime}(r)^{2}}{r}+2 v w^{\prime}(r) w^{\prime \prime}(r)\right) d r
$$

où le module de rigidité $D$ du disque est défini par

$$
D=\frac{E h^{3}}{12\left(1-v^{2}\right)}
$$

Les unités du module de rigidité sont $\mathrm{Nm}$ (Joules). II ne faut pas le confondre avec les autres modules de rigidité tels que $E$ ou $G$ dont les unités sont celles de la contrainte $\left(\mathrm{N} / \mathrm{m}^{2}\right)$. Remarquez la grande influence de l'épaisseur $\left(\mathrm{h}^{3}\right)$ de la plaque sur le module de rigidité (D). Cette influence va se répercuter sur l'établissement de la relation entre l'épaisseur (h) et la contrainte maximale. 
La fonction d'énergie potentielle totale s'écrit donc:

$$
\eta \bar{w}=\pi D \int_{0}\left(\tilde{w}^{\prime}(r)^{2}+\frac{\bar{w}^{\prime}(r)^{2}}{r}+2 v \bar{w}(r) \bar{w}^{\prime}(r)\right) d r-2 \pi \int_{0}^{\infty} q(r) \bar{w}(r) r d r
$$

où $\bar{w}$ représente toutes les déflections cinématiquement admissibles, c'està-dire celles qui satisfont les conditions frontières et qui ont une dérivée seconde continue. Selon le théorème de l'énergie potentielle minimum, la fonction $\backslash \hat{w}$ est stationnaire et possède une valeur minimale lorsque la fonction $\widehat{w}$ représente la déflection $w$.

Le théorème de l'énergie potentielle minimale permet de définir les équations différentielles en fonction de $w(r)$ de façon à ce que l'énergie potentielle totale soit considérée comme stationnaire. En fait, l'énergie potentielle totale est stationnaire lorsque la variation de la déflection est la déflection réelle $\widehat{w}$. La déflection réelle peut s'exprimer par :

$$
\widehat{w}(r)=w(r)+\delta w(r)
$$

où $\delta w(r)$ est la variation de la déflection. On remarque que $\delta w(r)=0$ pour n'importe quelle portion où la déflection est spécifiée par les conditions frontières. 
On peut réécrire la fonction d'énergie potentielle totale en fonction de $w(r)$ et de $\delta w(r)$, on obtient :

$$
V[w+\delta w]-V[w]=\int_{0}^{h_{0}}[\ldots] \delta w(r) d r+\text { conditions frontières }+ \text { termes d'ordre supérieurs }
$$

Pour obtenir une équation stationnaire, il est essentiel que le premier terme à droite de l'équation soit nul. Après plusieurs intégrations par partie, cette condition nous permet d'obtenir l'équation différentielle suivante :

$$
w^{m}(r)+\frac{2}{r} w^{m}(r)-\frac{1}{r^{2}} w^{\prime \prime}(r)+\frac{1}{r^{3}} w^{\prime}(r)=\frac{q(r)}{D}, 0<\mathrm{r}<b
$$

Pour résoudre cette équation, il faut considérer les conditions frontières. Premièrement, la distribution de charge appliquée est perpendiculaire au plan du disque, et il n'y a pas de moment appliqué au rebord du disque $(r=b)$ ni de force en cisaillement. Ceci nous permet d'écrire les équations de conditions frontières sous la forme:

$$
\begin{aligned}
V[w+\delta w]-V[w]=\ldots & +2 \pi D \delta w(b)\left\lfloor-b w^{\prime \prime}(b)-w(b)+\frac{1}{b} w^{\prime}(b)\right] \\
& +2 \pi D \delta w(b)\left[b w^{\prime \prime}(b)+v w^{\prime}(b)\right] \\
& +2 \pi D \delta w(0)\left[-b w^{\prime \prime}(r)-w(r)+\frac{1}{b} w^{\prime}(r)\right]_{r \rightarrow 0} \\
& +2 \pi D \delta w(0)\left[b w^{\prime \prime}(r)+v w^{\prime}(r)\right]_{r \rightarrow 0} \\
& + \text { termes d'ordre supérieur }
\end{aligned}
$$


Le théorème de l'énergie potentielle minimale oblige à ce que chacun des termes de premier ordre de $\delta w(r)$ soit égal à zéro. Les termes d'ordre supérieurs seront négligés.

Le cas qui nous intéresse est celui d'une plaque circulaire $(0<r<b)$ qui subit une distribution de pression uniforme $q(r)=-p$ et où le rebord $r=b$ est libre d'être en rotation. Ce qui implique les conditions frontières suivantes :

Par symétrie, la pente au centre de la plaque doit être nulle, ce qui implique que $\delta w^{\prime}(0)=0$. De plus, le déplacement au centre de la plaque est quelconque, ce qui a pour conséquence que le coefficient qui lui est associé doit être égal à zéro.

$$
-b w^{m}(r)-w(r)+\frac{1}{b} w^{\prime}(r) \rightarrow 0 \quad \text { lorsque } \mathrm{r} \rightarrow 0
$$

Pour une plaque simplement supportée sur les rebords, le déplacement au point $r=b$ est nul. Par contre la pente $w^{\prime}(b)$ n'est pas définie et c'est donc le coefficient qui lui est associé qui devra être égal à zéro.

$$
b w^{\prime \prime}(b)+v w^{\prime}(b)=0
$$


On obtient donc le système d'équations suivant à résoudre :

$$
\begin{aligned}
& w^{\prime \prime \prime}(r)+\frac{2}{r} w^{\prime \prime \prime}(r)-\frac{1}{r^{2}} w^{\prime \prime}(r)+\frac{1}{r^{3}} w^{\prime}(r)=\frac{q(r)}{D} \\
& w^{\prime}(0)=0 \\
& -b w^{\prime \prime \prime}(r)-w(r)+\frac{1}{b} w^{\prime}(r)=0 \\
& w(b)=0 \\
& b w^{\prime \prime}(b)+v w^{\prime}(b)=0
\end{aligned}
$$

L'écriture et la résolution du système d'équations à l'aide du logiciel « Mathématica » (voir annexe 1).

La solution du système d'équation pour la déflection est

$$
w(r)=-\frac{p}{64 D(1+v)}\left[\left(b^{2}-r^{2}\right)\left(b^{2}(5+v)-r^{2}(1+v)\right)\right]
$$

On obtient les contraintes maximales dans la plaque à partir des équations 4 évaluées au point $z=-h / 2$. On doit remarquer que la symétrie du problème permet d'obtenir exactement les mêmes valeurs de contrainte dans le cas où le disque serait supporté au centre et où les rebords sont libres de se déplacer en z. Ce dernier résultat sera important dans la section suivante lorsque nous analyserons la flexion des échantillons de glace. 


\subsubsection{Calcul des contraintes dans les échantillons circulaires de glace}

Les échantillons de glace sont de la forme d'un disque de rayon de $8,5 \mathrm{~cm}$ et d'une épaisseur variant entre 4 et $10 \mathrm{~mm}$. Les disques de glace sont déposés sur une plaque d'aluminium et soumis à une pression verticale engendrée par une onde de choc. Pour fabriquer ces échantillons, l'eau a été versée dans un plat de Petri où elle a été congelée à une température de $-4,5^{\circ} \mathrm{C}$. Cette façon de procéder ne produit pas des échantillons parfaitement plats. Ceux-ci sont plutôt très légèrement concaves ou convexes, en fait, comme les calculs vont le démontrer, cette déflection n'a pas besoin d'être importante pour produire un effet d'appui soit au centre ou sur les rebords. Cette particularité des échantillons justifie l'emploi du modèle de flexion développé dans la section précédente.

Pour la glace, le module de Young (E) est de $1 \times 10^{10} \mathrm{~N} / \mathrm{m}^{2}$ et le coefficient de Poisson (v) est de 0.33 . La contrainte de rupture en tension pour un taux de déformation supérieur $10^{-3} / \mathrm{s}$ est de $2,7 \times 10^{6} \mathrm{~N} / \mathrm{m}^{2}$. (Laforte et al.1994). L'équation de la distribution de charge choisie est $q(r)=-p(r-b) / b$ avec $p=175 \times 10^{3} \mathrm{~N}$. La justification de la distribution choisie sera apportée lorsque nous aborderons la discussion des résultats. 
Les calculs sont effectués avec l'aide du logiciel « Mathématica », à partir du modèle développé dans la section précédente avec les mêmes conditions frontières.

Les résultats des calculs sont présentés aux figures 13,14 et 15. La figure 13 montre la déflection $(w)$ en fonction du rayon pour un échantillon de $5 \mathrm{~mm}$ d'épaisseur. La déflection maximale de $0,156 \mathrm{~mm}$ est atteinte au centre de l'échantillon. Cette très faible valeur justifie l'utilisation du modèle pour d'écrire les appuis compte tenu de la forme des échantillons.

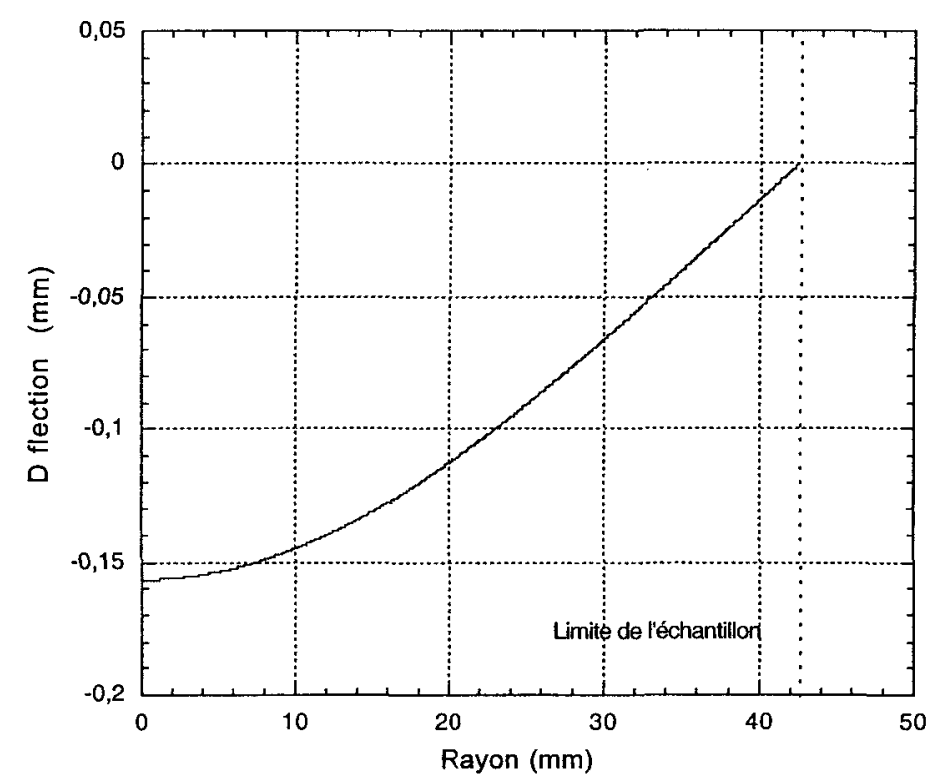

Figure 13: Déflection de l'échantillon en fonction du rayon. 
La figure 14 montre les contraintes maximales $\sigma_{\mathrm{rr}}$ et $\sigma_{\theta \theta}$ évaluées en $\mathrm{z}=-$ $\mathrm{h} / 2$ en fonction du rayon pour une épaisseur de $5 \mathrm{~mm}$. Les deux contraintes sont égales au centre $(r=0)$ ce qui est le résultat attendu compte tenu des symétries de l'échantillon. Par la suite, les deux contraintes s'écartent progressivement, la contrainte $\sigma_{\mathrm{rr}}$ diminue graduellement pour atteindre zéro au rebord du disque et la contrainte $\sigma_{\theta \theta}$ reste supérieure à $\sigma_{\mathrm{rr}}$ tout en diminuant pour atteindre la valeur d'environ $2,7 \times 10^{6} \mathrm{~N} / \mathrm{m}^{2}$. On devrait donc s'attendre à ce que la rupture de l'échantillon produise des fractures dans le sens du rayon.

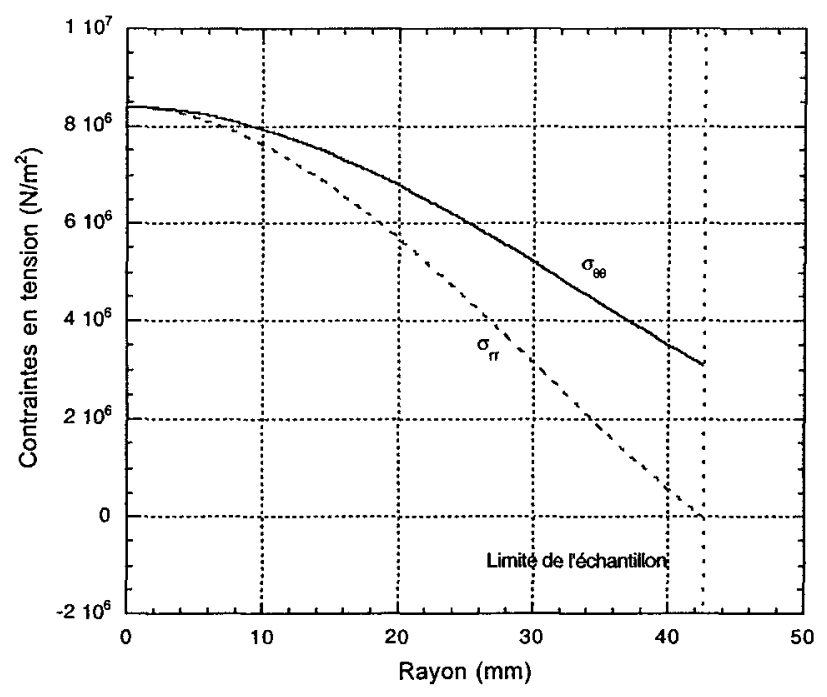

Figure 14: Contraintes $\sigma_{\mathrm{rr}}$ et $\sigma_{\theta \theta}$ en fonction du rayon d'une plaque de $5 \mathrm{~mm}$ d'épaisseur 


\section{Chapitre 3}

Montage expérimental et acquisition des données 


\section{Montage expérimental et acquisition des données}

\section{$3.1 \quad$ Introduction}

Dans ce chapitre, nous allons présenter les équipements utilisés pour l'expérimentation. Le montage est d'abord décrit de façon générale puis, chaque partie est expliquée de façon détaillée, soit : le circuit électrique, le dispositif de focalisation et le système d'acquisition de données.

\subsection{Montage expérimental}

Lors de la conception du montage, certaines balises ont été fixées : le système expérimental devait être capable de produire des ondes de choc dans l'air libre et de les focaliser sur des échantillons de glace. Il devait être transportable et il devait pouvoir fonctionner dans une chambre réfrigérée.

Pour produire les ondes de choc à l'air libre, nous avons choisi d'utiliser le claquage électrique à haut voltage. Cette méthode fournit moins d'énergie que l'utilisation d'explosifs, mais elle est plus contrôlable et sécuritaire. Les ondes de choc sont produites par la décharge d'un condensateur de $1 \mu \mathrm{F}$ à $10 \mathrm{kV}$ entre deux électrodes de tungstène. L'énergie électrique fournie est de 50 Joules et la focalisation des ondes de choc est obtenue par un réflecteur ellipsoïde. 
Dans le montage, les électrodes produisent au foyer primaire une onde de choc sphérique qui est focalisée au foyer secondaire par réflexion.

Tout le système de production et de focalisation de l'onde de choc est installé sur une structure mobile (voir Figure 15). L'axe de focalisation du réflecteur est orienté verticalement, ce qui permet d'obtenir un montage plus compact de même qu'une manipulation plus facile des échantillons. Le système de mesure et les échantillons sont placés en dessous du réflecteur sur un support indépendant. Le capteur est inséré dans une plaque d'aluminium de $10 \mathrm{~cm} \times 10$ $\mathrm{cm}$ qui est fixée sur un système de positionnement supporté par un banc d'optique. Le système de positionnement permet un déplacement vertical de $25 \mathrm{~cm}$. Cette plaque est utilisée comme support pour les échantillons de glace.

L'enlignement des échantillons sur l'axe central du réflecteur est assuré par un laser et des positionneurs millimétriques.

Les essais ont été effectués dans une chambre réfrigérée maintenue à $-4,5$ ${ }^{\circ} \mathrm{C}$. Le système de contrôle de l'alimentation électrique et d'acquisition des données était situé à l'extérieur de la chambre. 


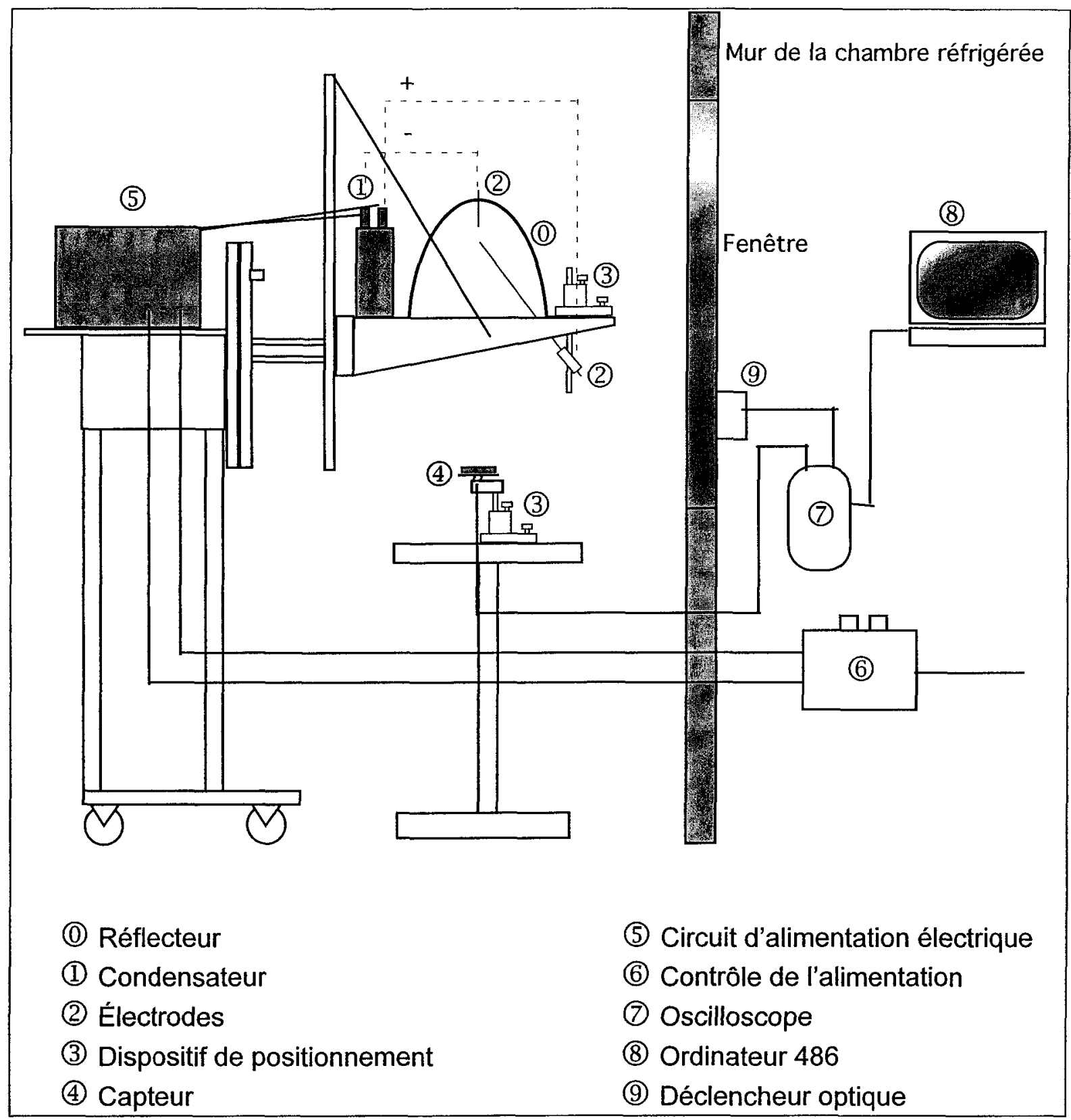

Figure 15: Montage utilisé pour la génération des ondes de choc. 


\subsubsection{Circuit électrique de l'éclateur}

Les pièces principales du circuit électrique de l'éclateur sont: un transformateur, un condensateur et l'éclateur (voir Figure 16). Le circuit d'alimentation électrique est conçu de façon à produire une décharge électrique minimale d'environ $10 \mathrm{kV}$. Le circuit construit est simple et robuste. La décharge se produit librement dans l'air entre 10 et $12 \mathrm{KV}$. Le condensateur de $1 \mu \mathrm{F}$ fournit une énergie de 50 joules à $10 \mathrm{kV}$ pour la production de la décharge électrique. La résistance R2 est utilisée pour contrôler un temps de charge d'environ 60 secondes et la résistance R3 est une résistance de sécurité qui est utilisée pour décharger le condensateur en environ 20 secondes lors de l'arrêt du système.

La problématique de la haute tension a imposé plusieurs précautions dans la conception et la fabrication du circuit. II fallait d'une part s'assurer que l'opération reste sécuritaire lors des manipulations et d'autre part, que les pièces d'équipements ainsi que les câbles qui relient les composantes soit organisés de façon à éviter les arcs accidentels et minimiser les pertes d'énergie par effet Joule. Le condensateur a été placé le plus près possible de l'éclateur auquel il est relié par des câbles rigides en cuivre de $1 \mathrm{~cm}$ de diamètre recouvert de $1,1 \mathrm{~cm}$ de téflon. De plus, tous les contacts ont été fermement serrés de façon à éviter la formation de micro-arcs électriques. 
Les électrodes sont constituées de deux tiges de cuivre de $4 \mathrm{~mm}$ de diamètre au bout desquelles ont été ajoutées des pointes de tungstène. Chaque pointe est aiguisée en un cône de façon à produire un chemin privilégié pour le passage de l'arc électrique lors du claquage, ce qui augmente la précision du positionnement au foyer primaire.

Un dispositif a été également construit pour assurer un déclenchement stable à environ $10 \mathrm{KV}$. Celui-ci consistait en une pointe placée près des électrodes et reliée à un dispositif piézoélectrique. Malheureusement, la décharge engendrait des interférences trop importantes dans la mesure de la pression. Le déclencheur a donc été retiré du montage et l'on a dû se contenter de décharges spontanées.

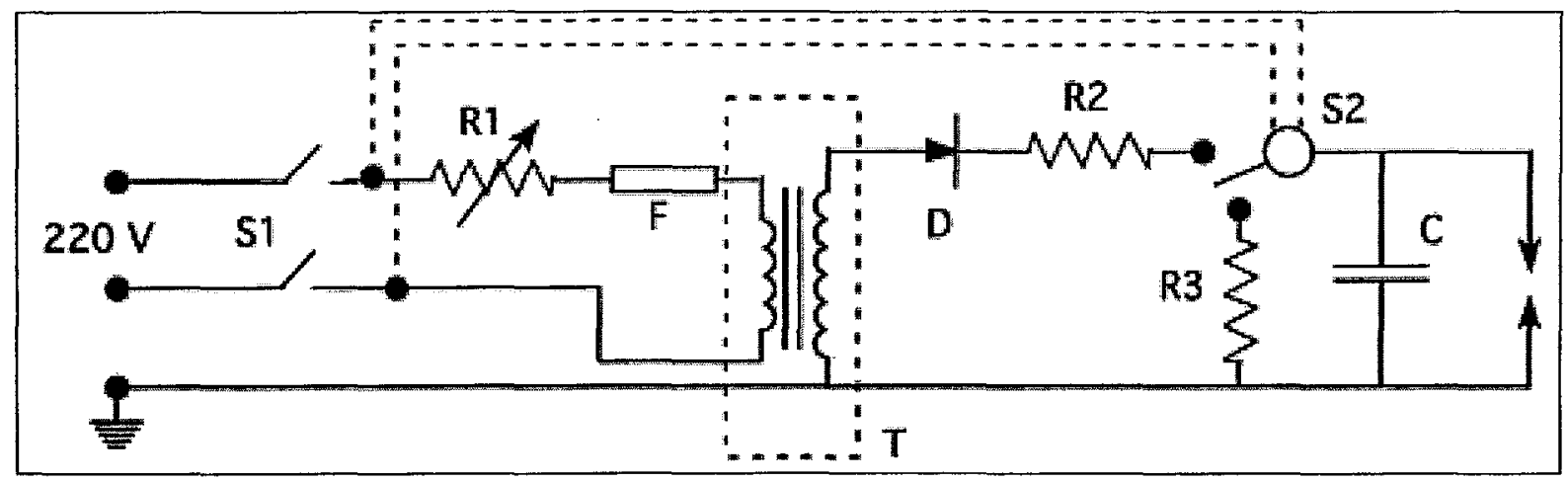

Figure 16: Schéma du circuit de l'éclateur 
Table 1 Composantes électriques du circuit du générateur d'ondes de choc

\begin{tabular}{|c|c|c|}
\hline Pièces & Caractéristique & Fabricant \\
\hline Interrupteur : S1 & Double polarité & ------- \\
\hline Interrupteur : S2 & Relais & --- \\
\hline Variac : R1 & $220 \mathrm{~V}$ & \\
\hline Résistance de & $10 \mathrm{M} \Omega$ & \\
charge : R2 & & \\
\hline Résistance de & $6800 \Omega 10 \%$ & \\
\hline décharge : R3 & Pw $10 \mathrm{watts}$ & \\
\hline Fusible : F & 5 ampères-250 V & \\
\hline Diode : D & $75 \mathrm{kV}$ & Semtech \\
\hline Condensateur : C & $1 \mathrm{mF}$ & \\
\hline Transformateur à & $220-1250 \mathrm{Volt}$ & \\
\hline
\end{tabular}




\subsubsection{Dispositif de focalisation}

Le dispositif de focalisation est constitué d'un réflecteur ellipsoïde. L'éclateur est situé au foyer primaire et la cible est placée au foyer secondaire. Trois réflecteurs ont été utilisés, que nous numéroterons respectivement 1,2 et 3 .

Le positionnement des électrodes est assuré par deux positionneurs millimétriques à deux degrés de liberté et d'un faisceau laser guide pour assurer une bonne focalisation.

À l'étape de la conception, il était difficile de prévoir quelles devaient être les caractéristiques du réflecteur de façon à maximiser le transfert d'énergie à l'interface air-glace. Comme point de départ, il a été décidé de faire construire un réflecteur possédant une distance focale d'environ $50 \mathrm{~cm}$ avec une excentricité de 0,8 comparable à celle utilisé dans les lithotriteurs Dormier (Cleveland et al., 2000). Par la suite, deux autres réflecteurs plus petits et avec une distance focale plus courte ont été utilisés. La description détaillée des réflecteurs se trouve dans les sections suivantes. 


\subsubsection{Réflecteur 1}

Le réflecteur 1 est une portion d'ellipse fabriquée en aluminium. La distance entre les foyers est de $48 \mathrm{~cm}$ et le diamètre intérieur de l'ouverture est de $35,8 \mathrm{~cm}$. Il a été poli en surface de façon à obtenir un fini miroir et possède une profondeur de $27 \mathrm{~cm}$. L'électrode négative passe par un trou percé au centre du réflecteur tandis que l'électrode positive passe par l'extérieur du réflecteur.

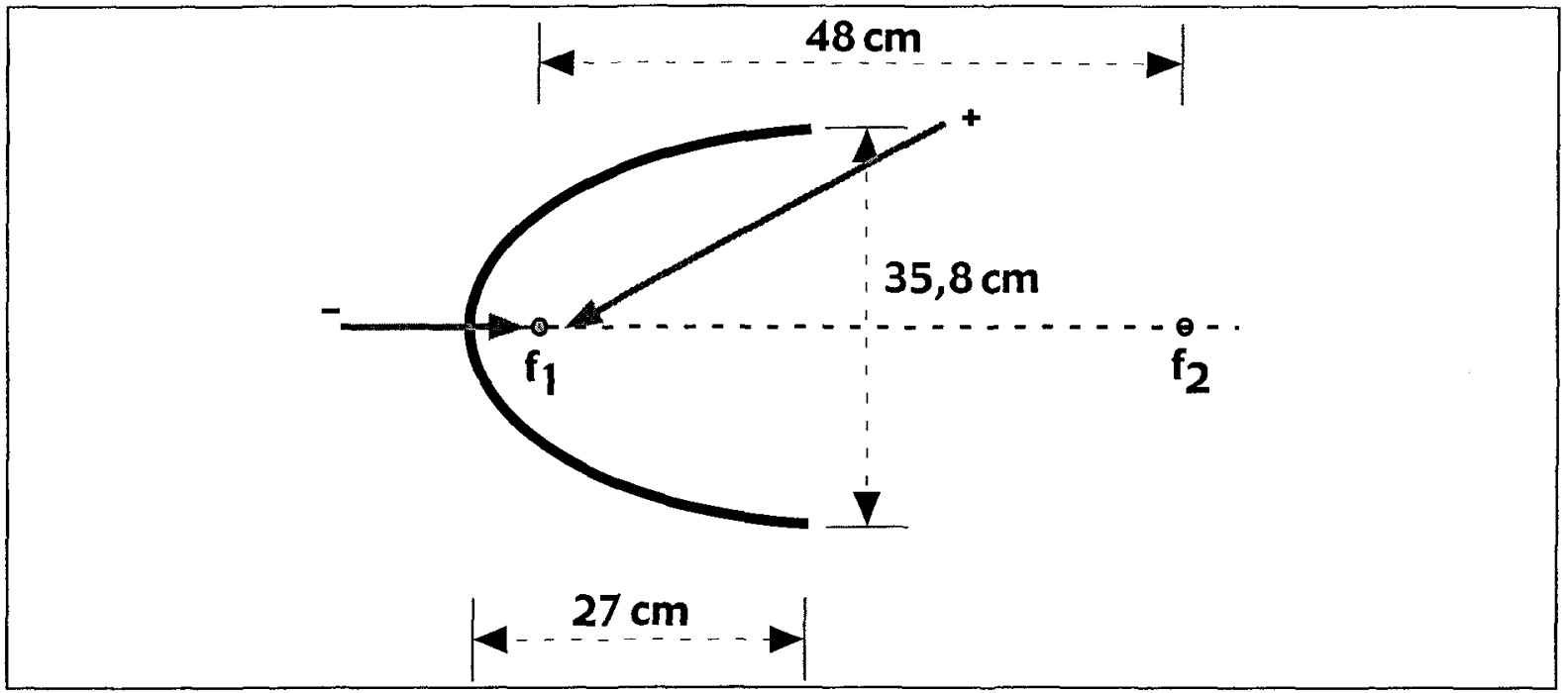

Figure 17: Réflecteur 1 


\subsubsection{Réflecteur 2}

Le réflecteur 2 est une ellipse dont une partie est allongée. La distance entre les foyers est de $12,2 \mathrm{~cm}$ et le diamètre intérieur de l'ouverture est de $8,2 \mathrm{~cm}$. Ce réflecteur est fait d'acrylique. Les deux électrodes sont insérées de chaque côté du réflecteur.

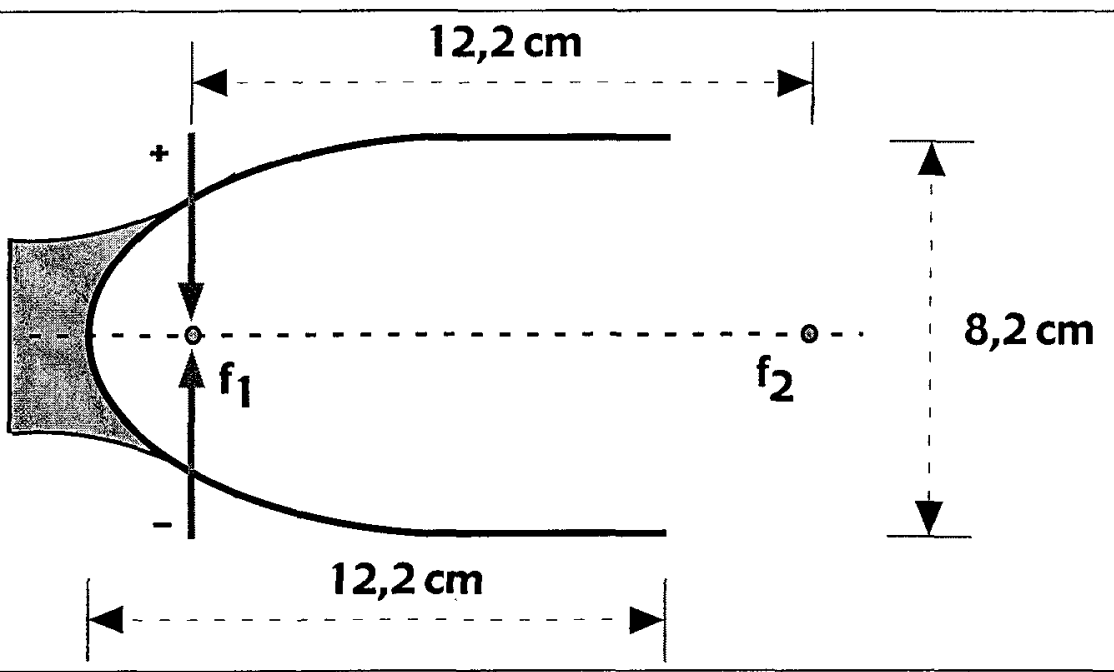

Figure 18: Réflecteur 2 


\subsubsection{Réflecteur 3}

Ce réflecteur est similaire au deuxième auquel la partie allongée a été retranchée. La distance entre les foyers est de $12,2 \mathrm{~cm}$ et le diamètre intérieur de l'ouverture est de $8,2 \mathrm{~cm}$. Ce réflecteur est fait d'acrylique. Les deux électrodes sont insérées de chaque côté du réflecteur.

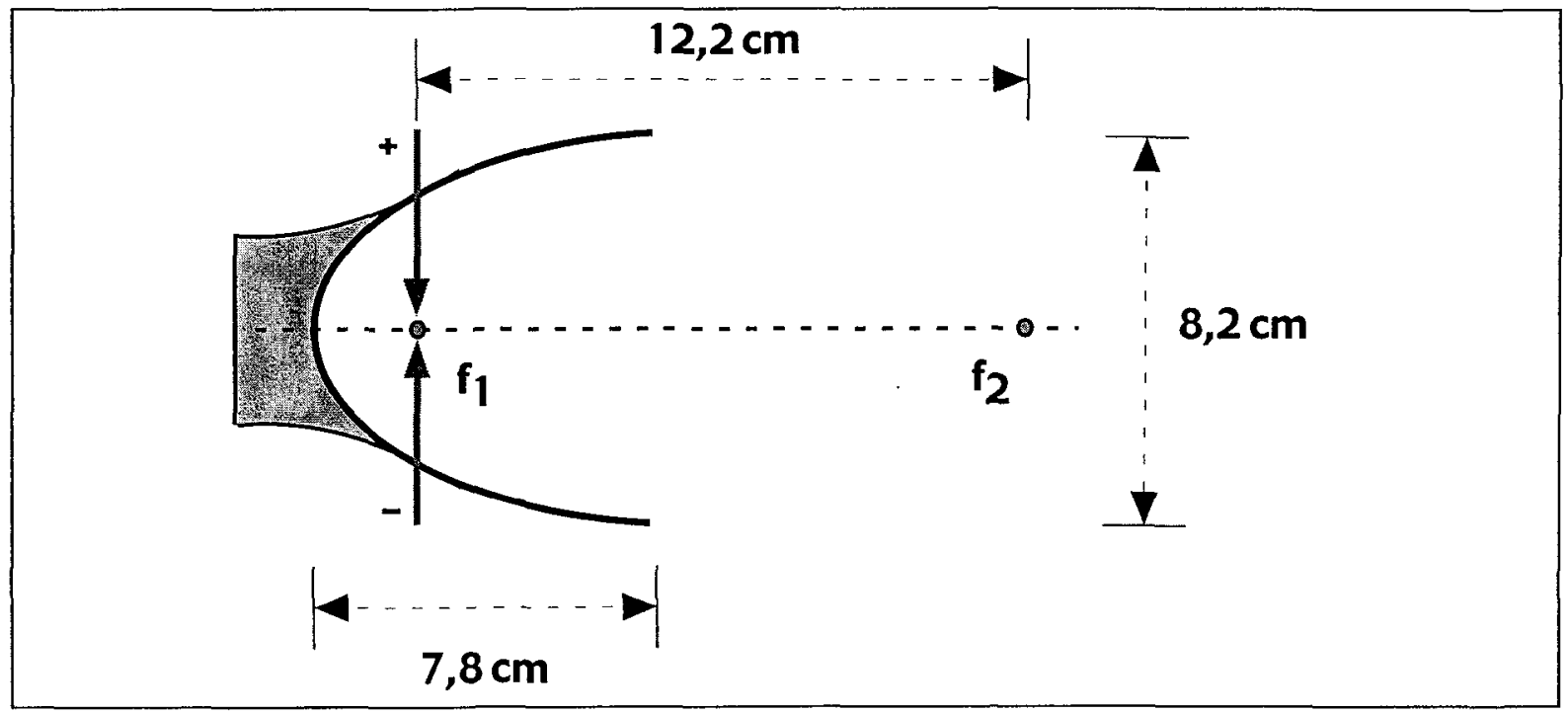

Figure 19: Réflecteur 3 


\subsection{Acquisition de données}

Les variations de pression engendrées par l'onde de choc est mesurée par un capteur de pression, modèle 102A de PCB Piezotronics, précalibré et spécialement conçu pour capter les ondes de choc. II est couplé à un conditionneur de signal (signal sensor conditionner ICP modèle 480E09).

Ce capteur est inséré dans la plaque d'aluminium servant de support aux échantillons. L'ensemble est supporté par un système de positionnement sur banc optique, celui-ci permettait un positionnement tant axial que radial.

L'acquisition des données est effectuée par un oscilloscope numérique Fluke 97 couplé à un ordinateur PC 486. Par la suite, les données sont transférées sur le logiciel Microsoft Excel, pour être classées et analysées. La synchronisation entre le capteur qui était à l'intérieur de la chambre réfrigérée et le système d'acquisition qui était situé à l'extérieur est assurée par un capteur optique installé sur la fenêtre de la chambre réfrigérée.

Une caméra vidéo a aussi servit à la prise de données. Le montage des images et des films a été réalisé sur ordinateur après leurs conversions en données numériques. 


\subsection{Montage pour le calcul de l'énergie de fracture}

Un petit montage a été réalisé pour déterminer de l'énergie de fracture en mode quasi statique des échantillons de glace. Le montage est constitué d'un solénoïde qui soutient une bille d'acier de 11,9 grammes. Celui-ci est installé sur un support ajustable en hauteur et les mesures de hauteur sont assurées par une règle précise au demi-millimètre.

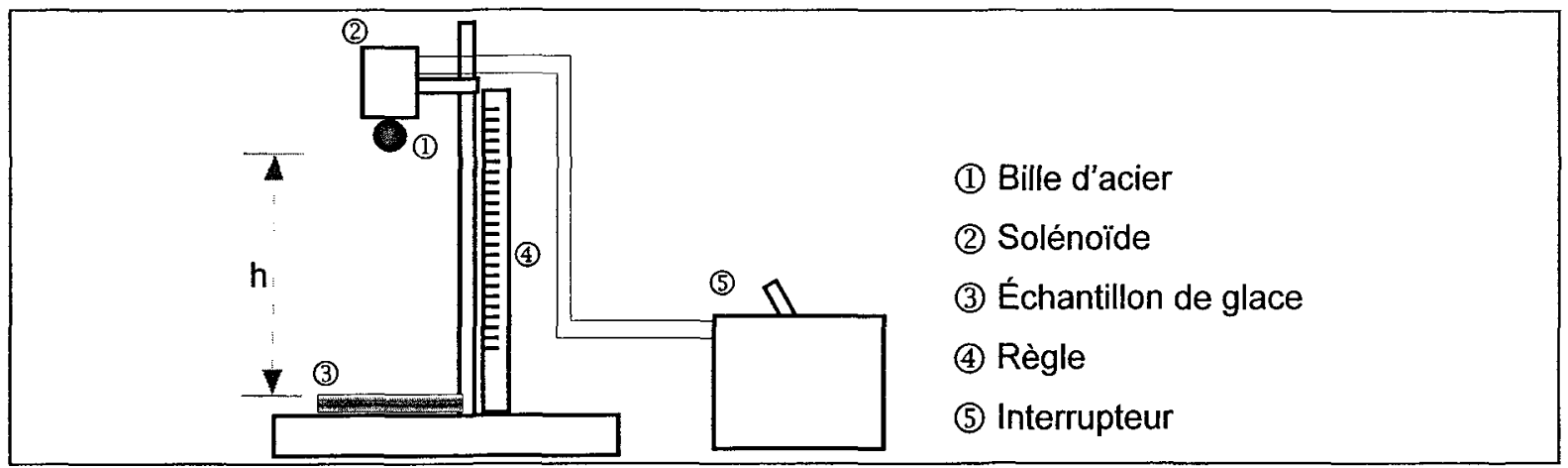

Figure 20: Montage utilisant une bille pour la briser les échantillons de glace.

L'échantillon est simplement déposé sur le support et la bille dans sa chute percute le centre de l'échantillon de glace qui se brise. La hauteur mesurée sert à calculer l'énergie nécessaire à la fracture $\left(E_{f r}\right)$.

$$
E_{f r}=m g h
$$

où $\mathrm{m}$ est la masse de la bille en $\mathrm{kg}, \mathrm{g}$ est l'accélération gravitationnelle $(9,8$ $\mathrm{m} / \mathrm{s}^{2}$ ) et $\mathrm{h}$ est la hauteur parcourue par la bille avant de frapper l'échantillon. 


\section{Chapitre 4}

Résultats expérimentaux 


\section{Résultats expérimentaux}

\subsection{Caractérisation des réflecteurs}

Pour le réflecteur 1 , la pointe de pression maximale atteinte est de $41 \mathrm{kPa}$, ce qui est insuffisant pour provoquer la rupture des échantillons. Par conséquent, celui-ci c'est révélé non-utilisable pour les fins de notre étude. Pour les réflecteurs 2 et 3 , deux séries de mesures de pression ont été effectuées. La première série de mesures a été prise à des intervalles de $0,5 \mathrm{~cm}$ sur l'axe central. Cette série a permis de calculer la vitesse du front d'onde et de déterminer la position où l'intensité de la focalisation est maximale. La deuxième série de mesure a été prise transversalement (radialement) avec des intervalles de $0,5 \mathrm{~cm}$, au point maximal de pression, de façon établir la distribution de pression auquel sont soumis les échantillons de glace.

La température de l'air était de $-4,5^{\circ} \mathrm{C}$ et l'humidité relative de $50 \%$. On obtient la vitesse du son à cette température par :

$$
\begin{aligned}
& V=331,3+0,6 T \\
& \text { où T est en }{ }^{\circ} \mathrm{C} \\
& \text { On obtient : } V=328,6 \mathrm{~m} / \mathrm{s} \text { à }-4,5^{\circ} \mathrm{C}
\end{aligned}
$$




\subsubsection{Mesure et marges d'erreurs}

\subsubsection{Erreur sur la mesures de la pression.}

Le déclenchement de l'arc électrique ne pouvant pas être contrôlé par un dispositif de déclenchement qui aurait pu assurer un déclenchement de l'arc à voltage constant, les mesures ont dû être sélectionnées et seules les mesures des pressions engendrées par le déclenchement de l'arc à $10 \pm 0,4 \mathrm{kV}$ ont été conservées. Ce qui correspond à une erreur relative de $4 \%$ sur le voltage de déclenchement. À cette erreur on doit ajouter la variation de la pression, car un même voltage de déclenchement peut produire des pressions différentes. Cette valeur est difficile à évaluer, mais l'examen de l'écart entre les résultats nous permet de l'estimer à environ $15 \%$. Donc l'erreur relative sur la mesure des pressions est de $19 \%$.

\subsubsection{Erreur sur la position axiale et radiale.}

Les positions du capteur ont été mesurées à l'aide d'une règle dont la plus petite division est le millimètre. Suivant la règle de la moitié de la plus petite division on obtient $\pm 0,5 \mathrm{~mm}$. Pour la distance focale de $122 \mathrm{~mm}$ du réflecteur 2 , on obtient une erreur relative de $0,4 \%$. Ce qui est négligeable. 


\subsubsection{Erreur sur la mesure du temps.}

Les temps mesurés sont de l'ordre de la milliseconde et la précision de la mesure est de l'ordre de la microseconde. L'erreur sur la mesure du temps est donc négligeable.

\subsubsection{Erreur sur la dimension des échantillons}

Le diamètre des échantillons était de $85 \mathrm{~mm}$ et il a été mesuré avec une précision de $\pm 0,5 \mathrm{~mm}$. Ce qui nous donne une erreur relative d'environ $0,6 \%$. Pour l'épaisseur, la mesure a été effectuée avec un pied à coulisse dont la précision est de 0,01 $\mathrm{mm}$. Par contre, la concavité des échantillons augmente l'imprécision de la mesure que l'on peut évaluer à $\pm 0,1 \mathrm{~mm}$. On obtient pour la plus petite épaisseur (3,7 mm ), une erreur relative d'environ $2,7 \%$. 


\subsubsection{Caractérisation du réflecteur 2}

Le réflecteur 2 a été caractérisé au niveau radial et axial à partir d'un voltage de décharge de $10 \pm 0,4 \mathrm{kV}$. La Figure 21 montre une pointe de pression d'environ $360 \mathrm{kPa}$ qui est atteinte à 1,17 fois la distance entre les foyers.

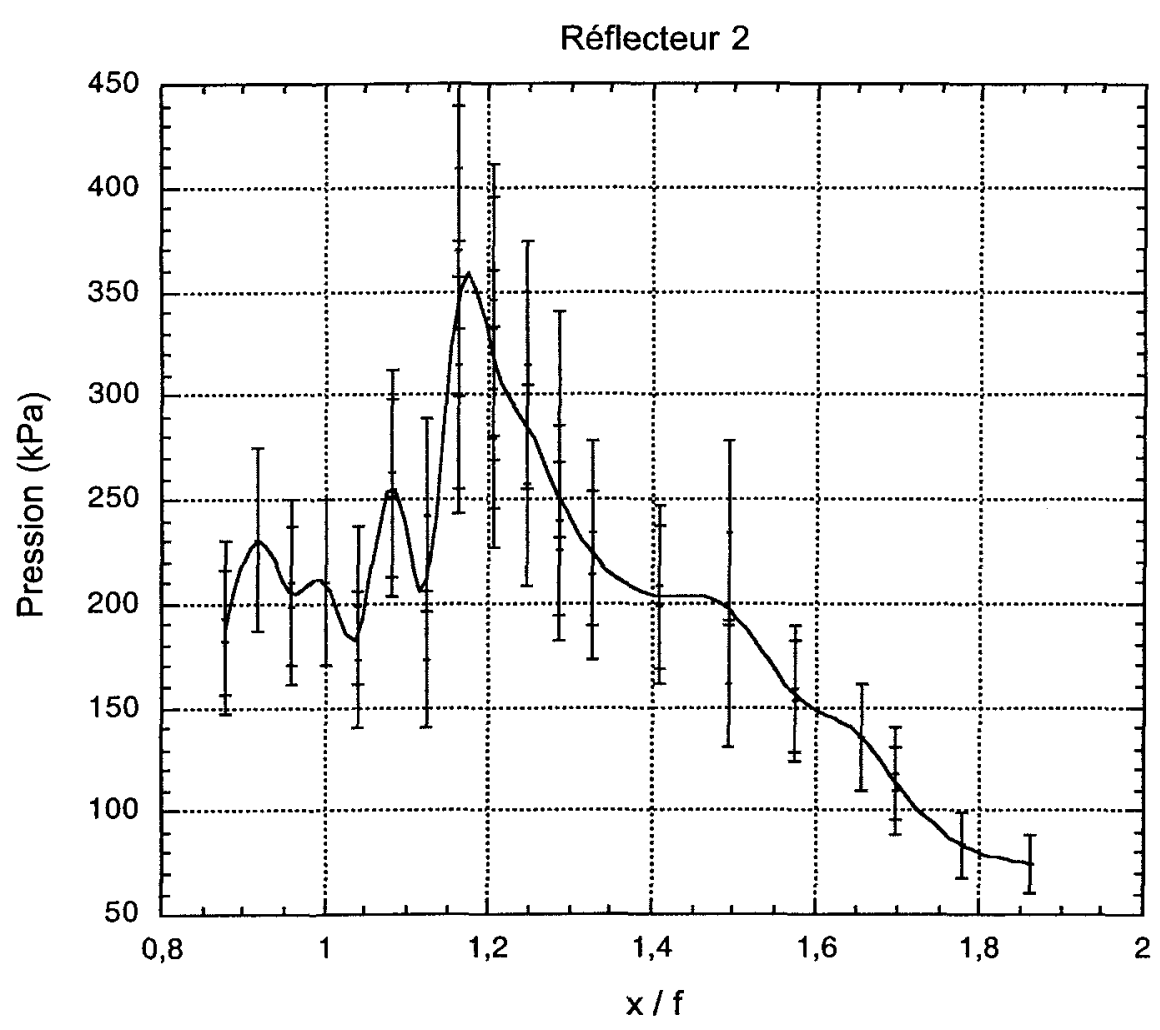

Figure 21: Pression en fonction de la distance axiale normalisée sur la distance focale (f) pour le réflecteur 2 
La Figure 22 représente la distribution radiale du réflecteur 2 évalué a 1,17 fois la distance entre les foyers. La pression maximale est d'environ $350 \mathrm{kPa}$ et la prise de mesure montre un léger décalage du pic de pression par rapport au centre qui reste toutefois dans les marges d'erreurs de l'expérimentation.

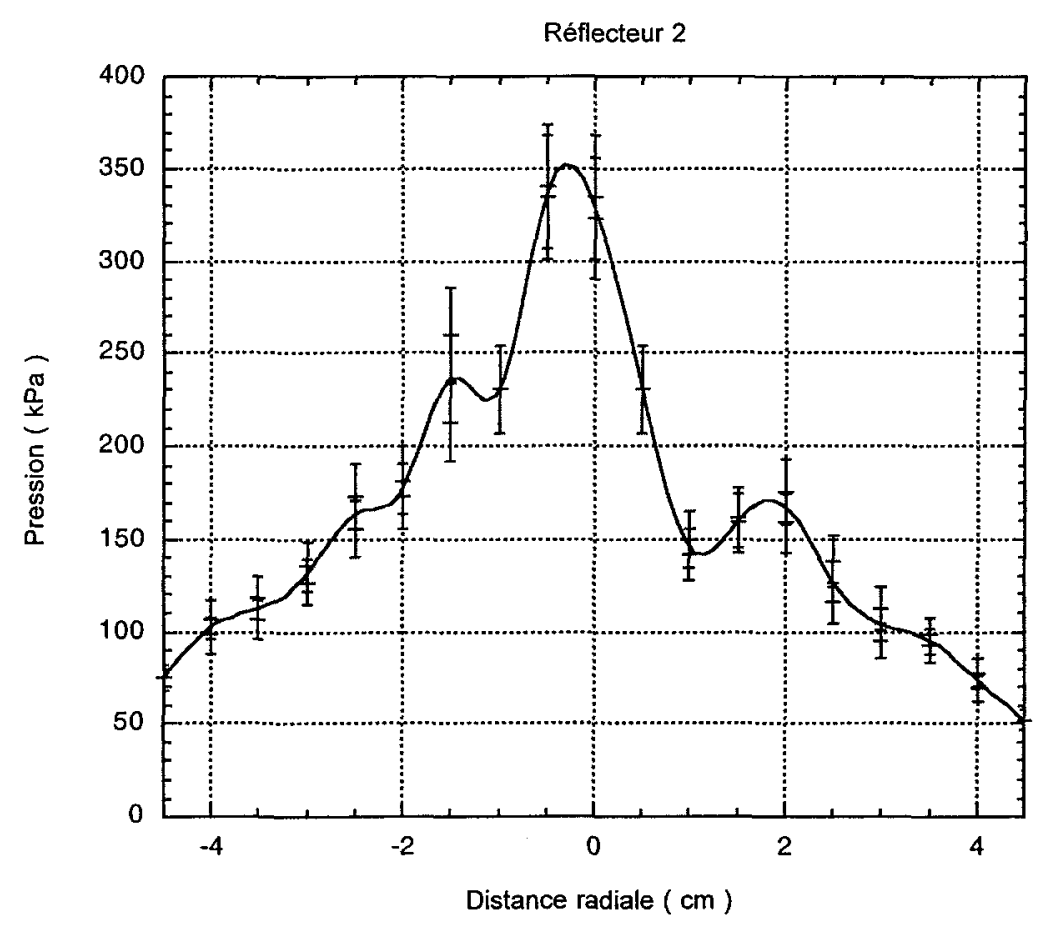

Figure 22: Distribution radial de pression du réflecteur 2 évalué à $x / f=1,17$ 
Les mesures de la vitesse de l'onde de choc ont été effectuées en variant la position axiale du détecteur par saut de $0,5 \mathrm{~cm}$. L'intervalle de temps a été obtenu en mesurant le temps entre les pics de pression, le premier pic servant de point de référence. La Figure 23 montre une vitesse de $447,2 \mathrm{~m} / \mathrm{s}$ et comme la vitesse du son à $-4,5^{\circ} \mathrm{C}$ est de $328,6 \mathrm{~m} / \mathrm{s}$, on obtient un nombre de Mach de 1,36 .

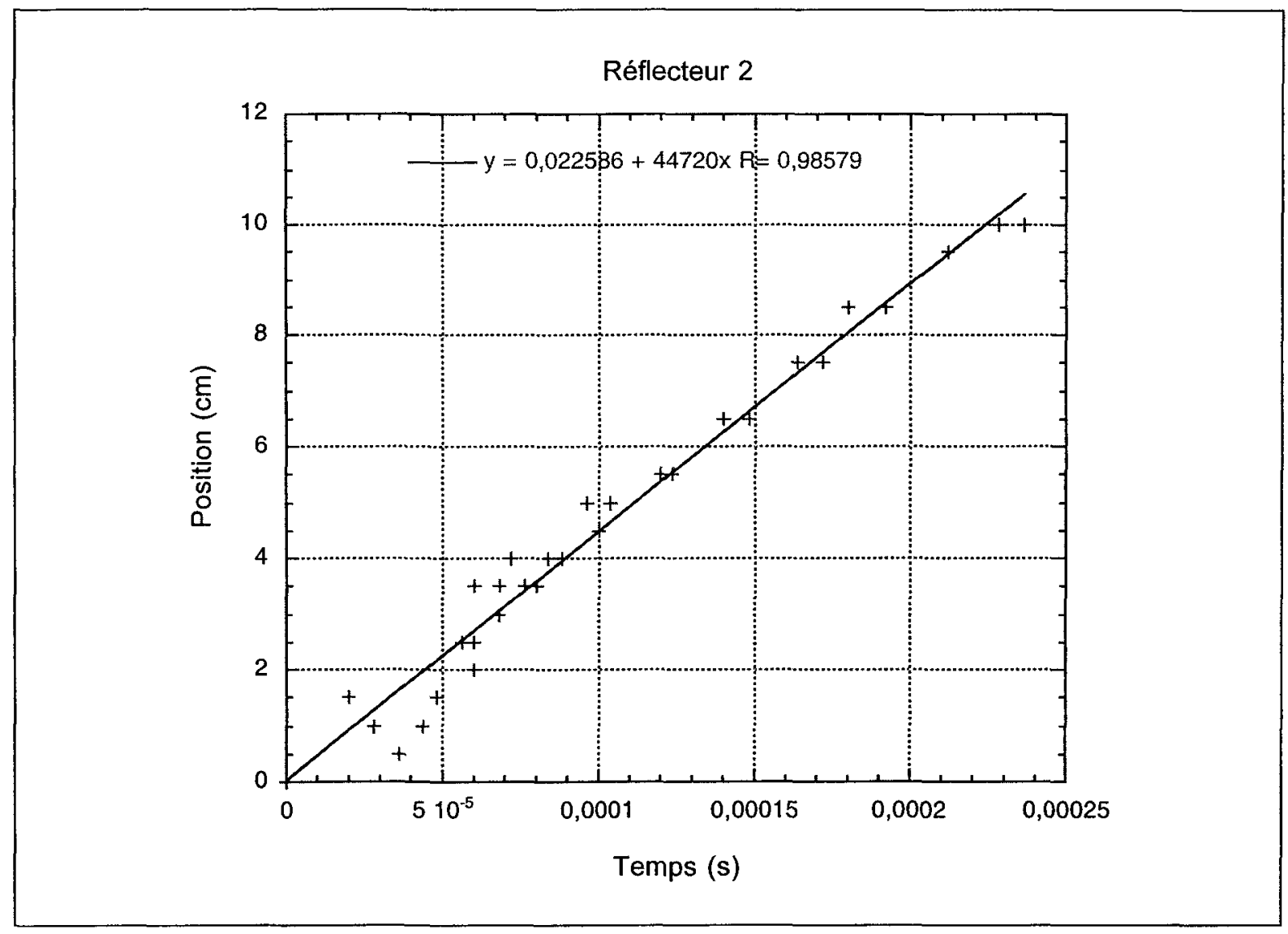

Figure 23: Vitesse du front de l'onde de choc pour le réflecteur 2 


\subsubsection{Caractérisation du réflecteur 3}

Le réflecteur 3 a été caractérisé au niveau radial et axial à partir d'un voltage de décharge de $10 \pm 0,4 \mathrm{kV}$. La Figure 24 montre une pression maximale d'environ $300 \mathrm{kPa}$ qui est atteinte à 0,8 fois la distance entre les foyers.

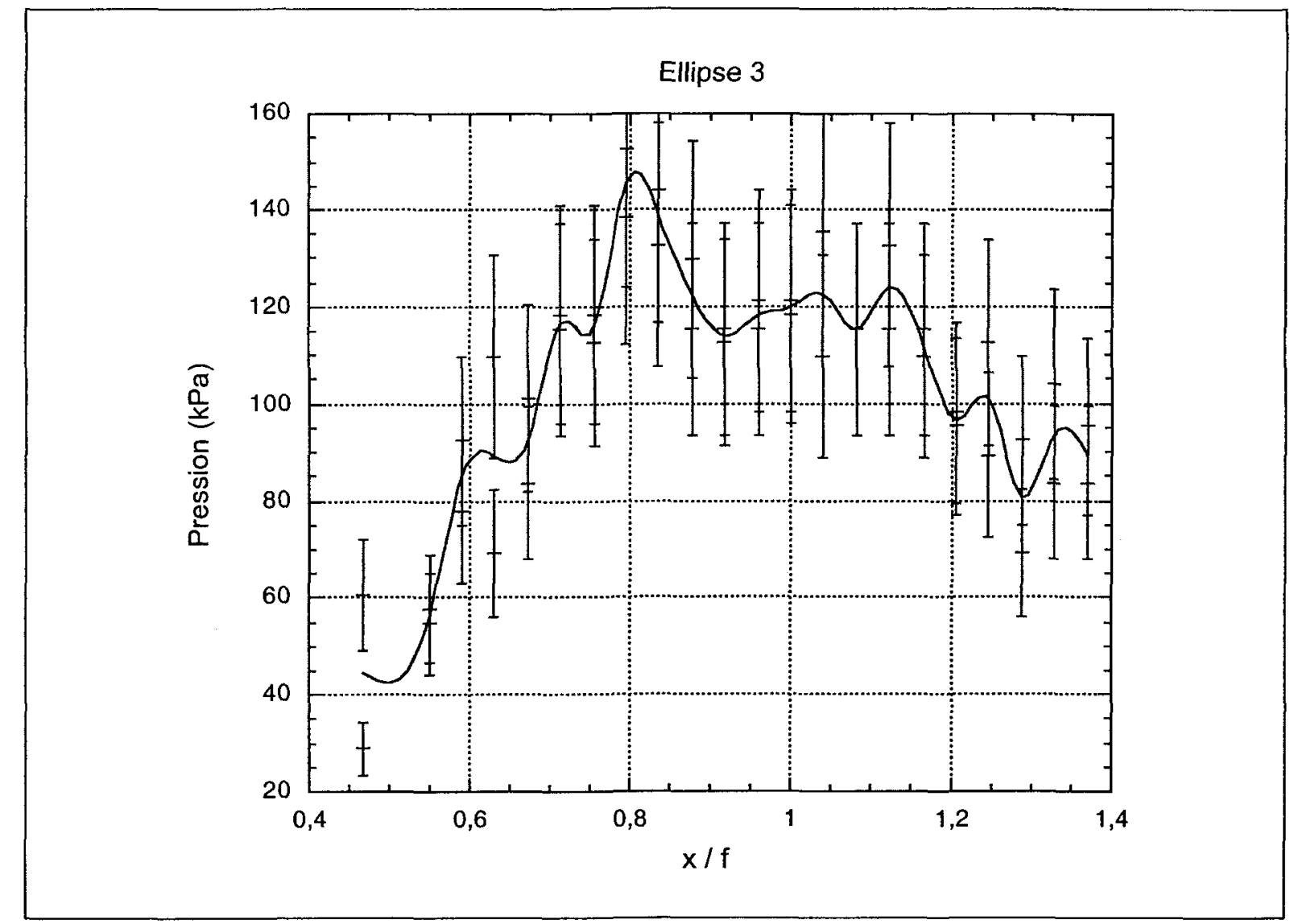

Figure 24: Pression en fonction de la distance axiale normalisée sur la distance focale (f) pour le réflecteur 3 
La Figure 25 représente la distribution radiale du réflecteur 3 évalué à 0,8 fois la distance entre les foyers. La pression maximale est d'environ $340 \mathrm{kPa}$ et la prise de mesure montre un léger dédoublement du pic central de pression.

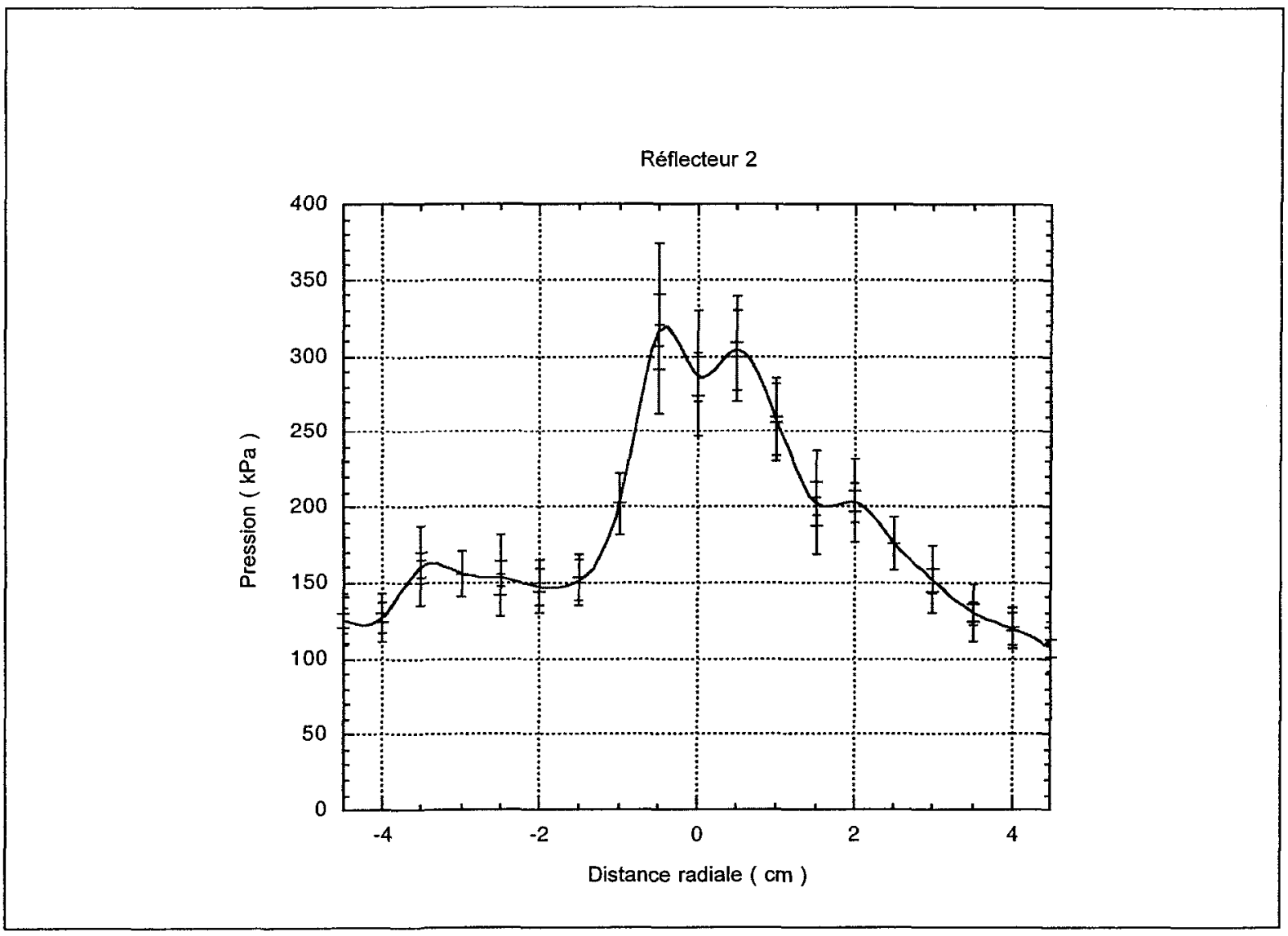

Figure 25: Distribution radiale de pression du réflecteur 2 évalué à $x / f=0,8$ 
Les mesures de vitesse de l'onde de choc ont aussi été effectuées en variant la position axiale du détecteur par saut de $0,5 \mathrm{~cm}$. L'intervalle de temps a été obtenu en mesurant le temps entre les pics de pression, le premier pic servant de point de référence. La Figure 26 montre une vitesse de $462,8 \mathrm{~m} / \mathrm{s}$ et comme la vitesse du son à $-4,5^{\circ} \mathrm{C}$ est de $328,6 \mathrm{~m} / \mathrm{s}$, on obtient un nombre de Mach de 1,40 .

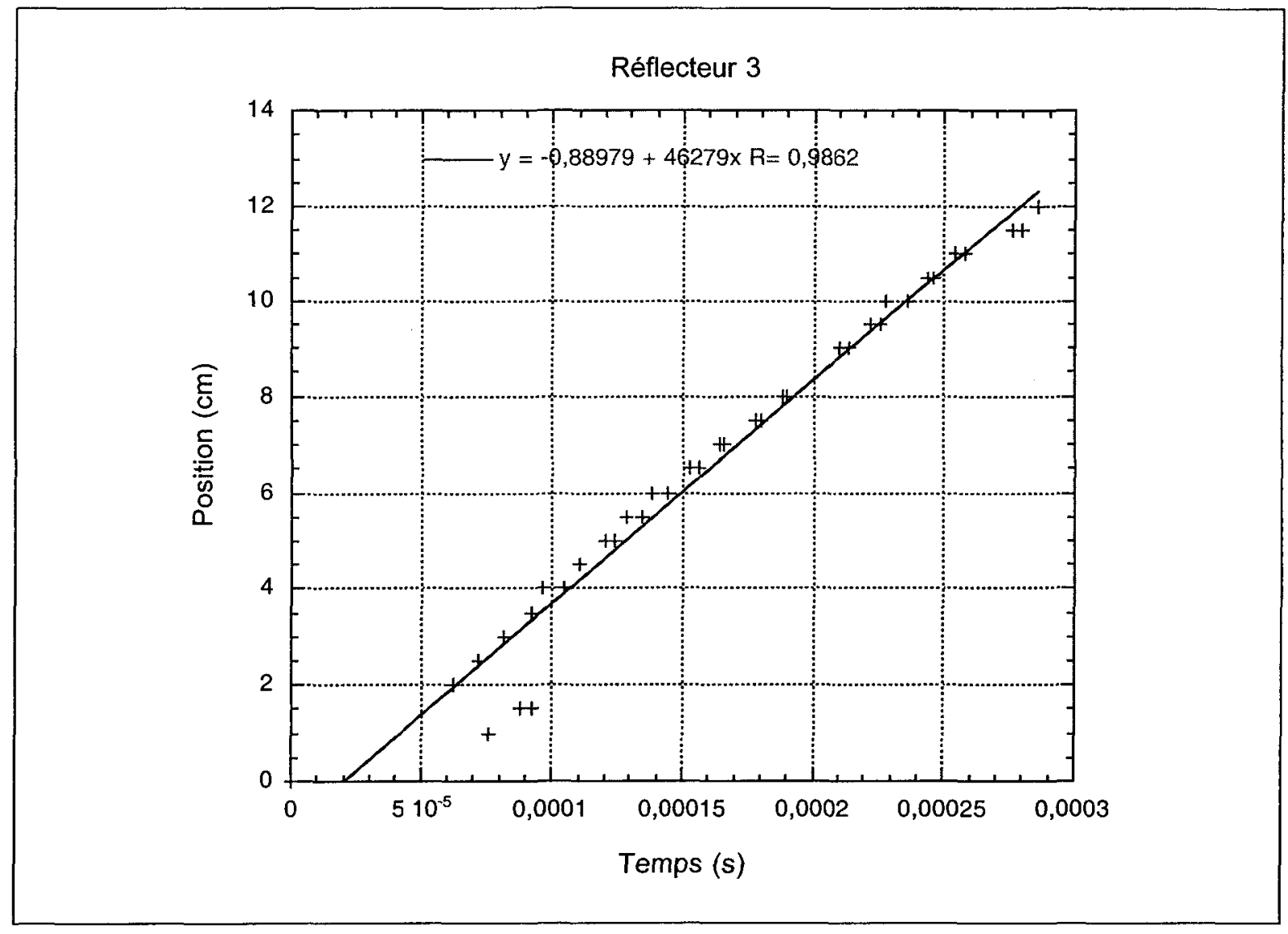

Figure 26: Vitesse du front de l'onde de choc pour le réflecteur 3 


\subsubsection{Sommaire de la caractérisation des réflecteurs}

Tableau 2; Sommaire des résultats sur la caractérisation des réflecteurs.

\begin{tabular}{|c|c|c|c|}
\hline Réflecteurs & $\begin{array}{c}\text { Position du maximum } \\
\text { de pression } \\
\text { normalisée au foyer }\end{array}$ & $\begin{array}{c}\text { Pression maximale } \\
\text { moyenne } \\
(\mathrm{kPa})\end{array}$ & Nombre de Mach \\
\hline 1 & ----- & 4,1 & $-\cdots-36$ \\
\hline 2 & 1,17 & 355 & 1,36 \\
\hline 3 & 0,80 & 320 & 1,40 \\
\hline
\end{tabular}

Les deux réflecteurs produisent des ondes de pression d'intensité semblable (voir tableau ci-dessus) qui se propagent à des vitesses pratiquement égales $(1,36$ et 1,40 Mach). Les différences sont mineures et restent dans la précision de l'expérimentation.

Par contre, on remarque que la position axiale du maximum de pression, est plus éloignée pour le réflecteur $2(x / f=1,17)$ que pour le réflecteur $3(x / f=0,80)$. Ce déplacement provient probablement de la différence de dimensions entre les deux réflecteurs. Celle-ci tient essentiellement à ce que le réflecteur 2 possède une partie allongée et droite de $4,4 \mathrm{~cm}$ au bout de la partie elliptique. (voir Figure 18 et Figure 19). 


\subsection{Caractéristiques des échantillons de glace}

Les échantillons sont obtenus par congélation à une température de $-4,5^{\circ} \mathrm{C}$ de l'eau déminéralisée dans des plats de Petrie de $8,5 \mathrm{~cm}$ de diamètre. Les échantillons sont par la suite démoulés et posés sur une plaque d'aluminium pour être soumis aux ondes de choc. L'épaisseur des échantillons varie de 4 à $10 \mathrm{~mm}$ avec une densité moyenne de 0,88 . La densité a été mesurée par différence de poids dans un liquide de densité connu. La densité théorique de la glace pure est de 0,917 ; les caractéristiques des échantillons correspondent donc a du verglas contenant des bulles. 


\subsection{Résultats des essais de bris de glace}

Les résultats, présentés dans les pages suivantes, sont illustrés à partir des photographies extraites des films vidéo numérisées. La figure 27 présente des exemples de résultats obtenus à $-4,5^{\circ} \mathrm{C}$ sur des échantillons de verglas de 3,7 $\mathrm{mm}, 5,2 \mathrm{~mm}$ et de $6,2 \mathrm{~mm}$ cassés avec le réflecteur 2 . Une seule onde de choc a été nécessaire pour produire la fracture des échantillons. On distingue nettement une fracture qui se propage dans le sens radial du disque de glace. On peut voir sur les images montrées à la figure 28 que le réflecteur 3 produit à $-4,5^{\circ} \mathrm{C}$ le même type de fracture avec des échantillons de verglas et d'épaisseurs comparables $(3,1 \mathrm{~mm}, 4,4 \mathrm{~mm}$ et $5,9 \mathrm{~mm})$. Comme pour le réflecteur 2 , les échantillons se brisent après le passage d'une seule onde de choc.

Les figures 29 à 35 représentent deux cas limites où les échantillons sont d'une épaisseur de 9,2 $\mathrm{mm}$ et de 9,9 $\mathrm{mm}$. 


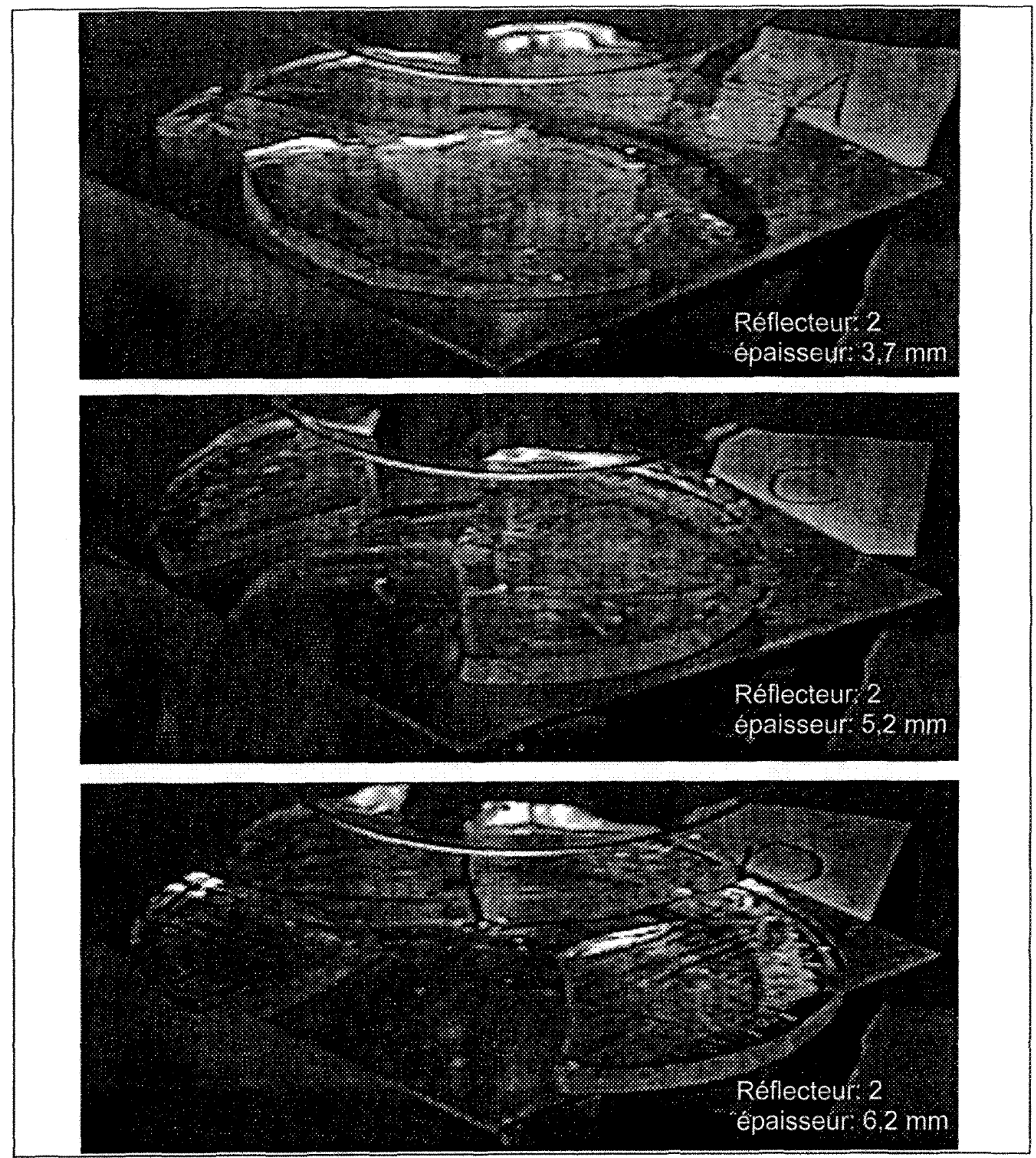

Figure 27: Fractures des échantillons de $3,7 \mathrm{~mm}, 5,2 \mathrm{~mm}$ et $6,2 \mathrm{~mm}$ avec le réflecteur 2. 


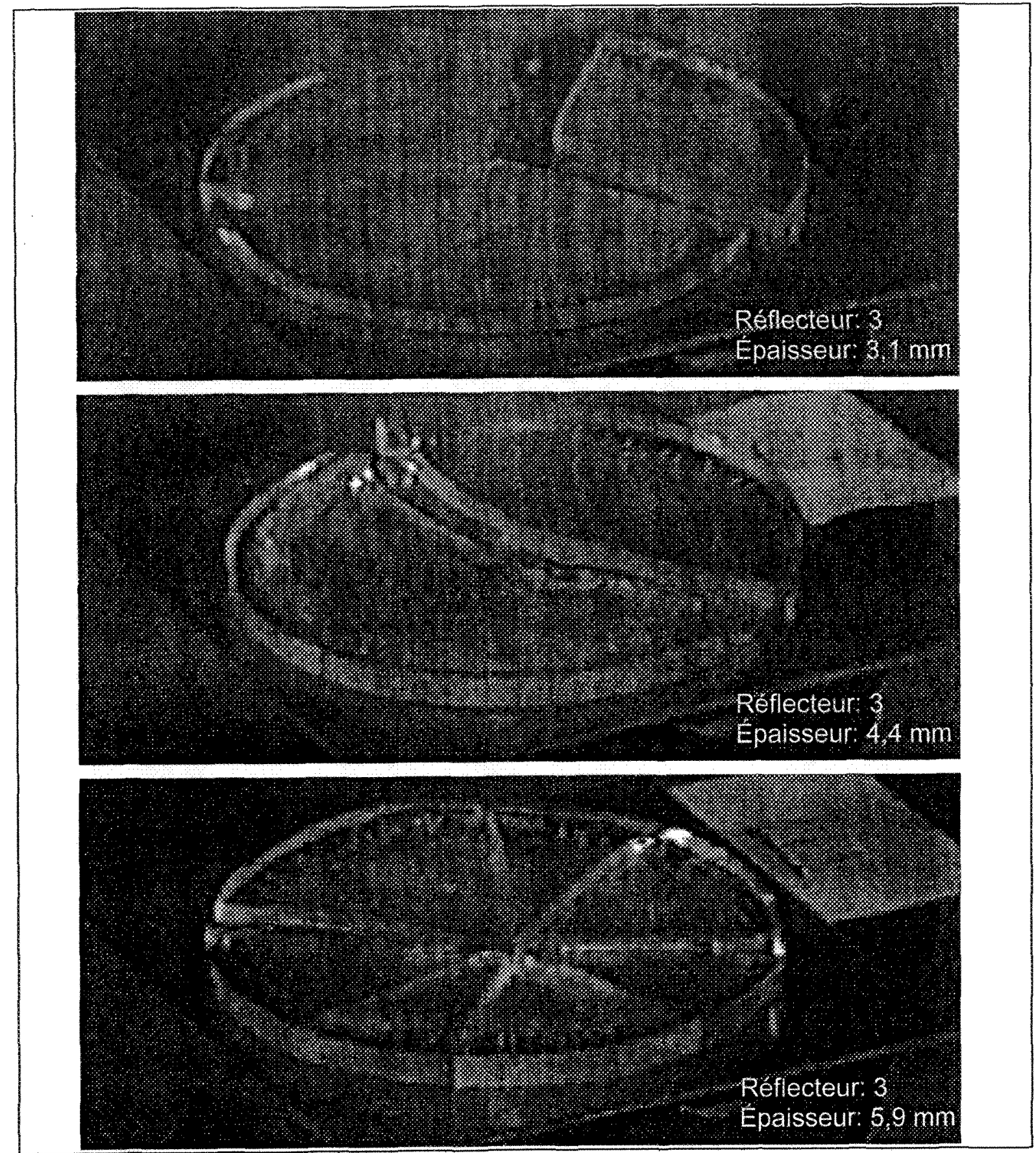

Figure 28: Fractures des échantillons de $3,1 \mathrm{~mm}, 4,4 \mathrm{~mm}$ et $5,9 \mathrm{~mm}$ avec le réflecteur 3 
Les figures 29 à 33 présentent l'évolution de la fracture d'un échantillon de $9,2 \mathrm{~mm}$ soumis à une succession d'onde de choc produite par le réflecteur 2. La fracture complète de l'échantillon en cinq morceaux se produit après quatre coups. La figure 29 nous présente l'échantillon avant le premier coup. Dans la figure 30, après le premier coup, on distingue la formation de cinq fissures qui sont indiquées par des flèches.

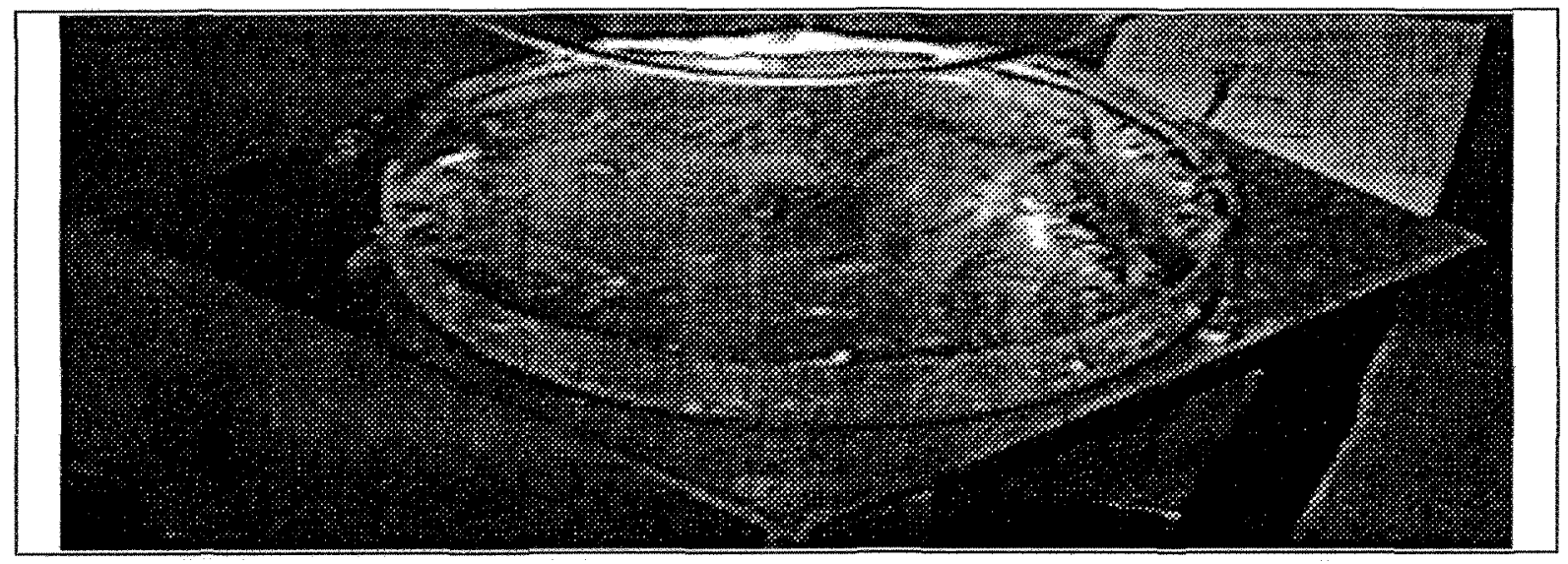

Figure 29: Échantillon de verglas d'une épaisseur $9,2 \mathrm{~mm}$ avec réflecteur 2 : aucun coup

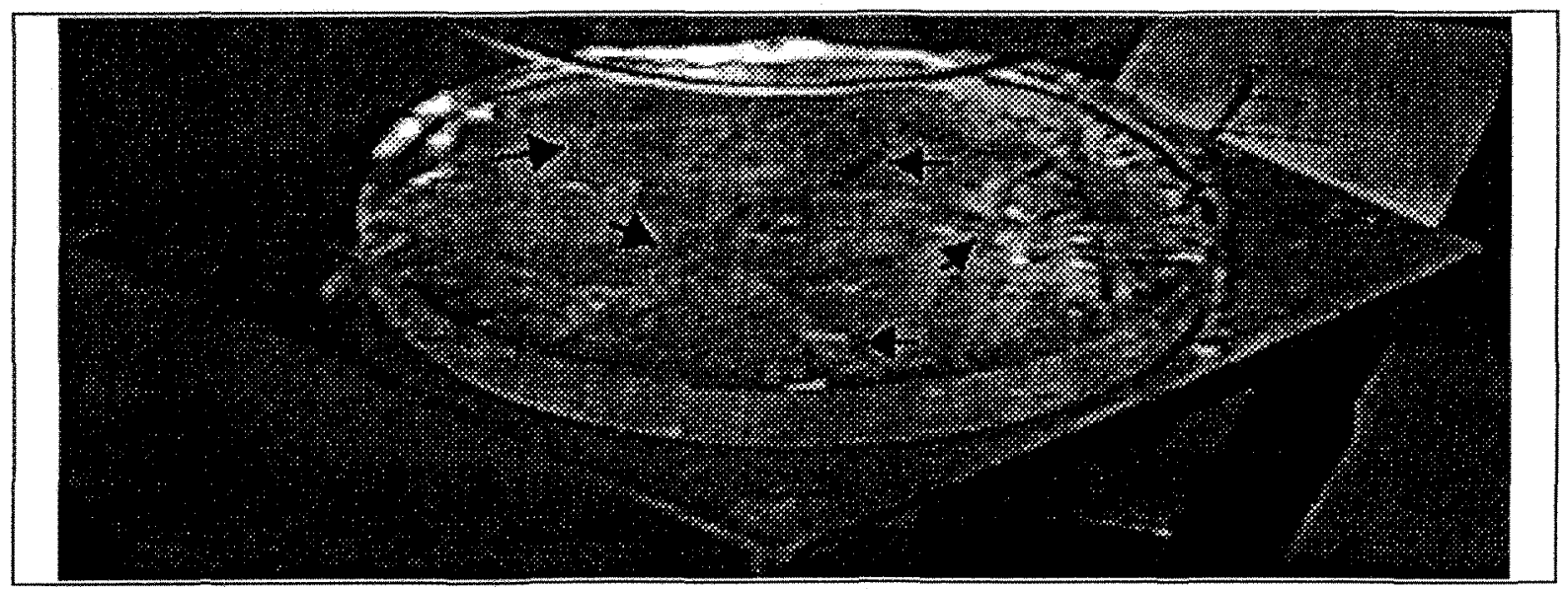

Figure 30: Échantillon de verglas d'une épaisseur 9,2 $\mathrm{mm}$ avec réflecteur 2 : premier coup 
La figure 31 montre l'échantillon après le deuxième coup, les fissures se propagent et elles sont clairement visibles. Au troisième coup (figure 32), deux morceaux se détachent du disque et l'échantillon est fracturé.

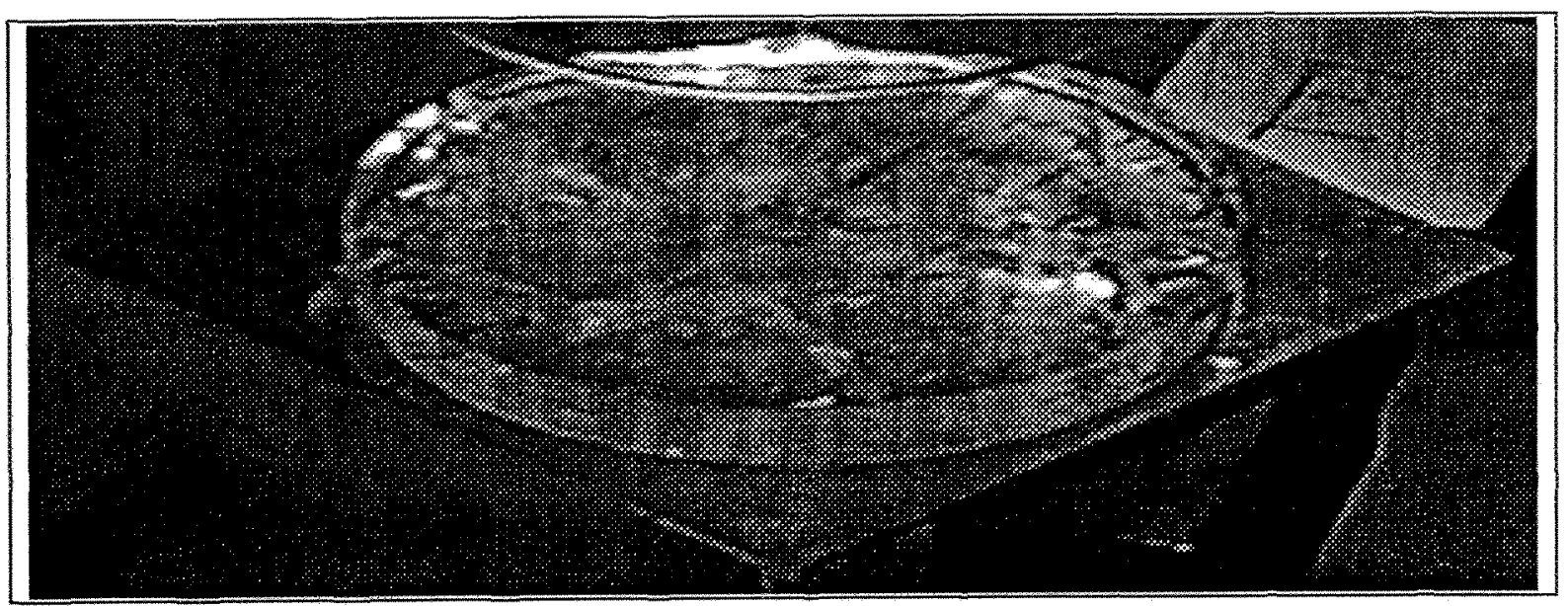

Figure 31: Échantillon de verglas d'une épaisseur $9,2 \mathrm{~mm}$ avec réflecteur $2:$ deuxième coup

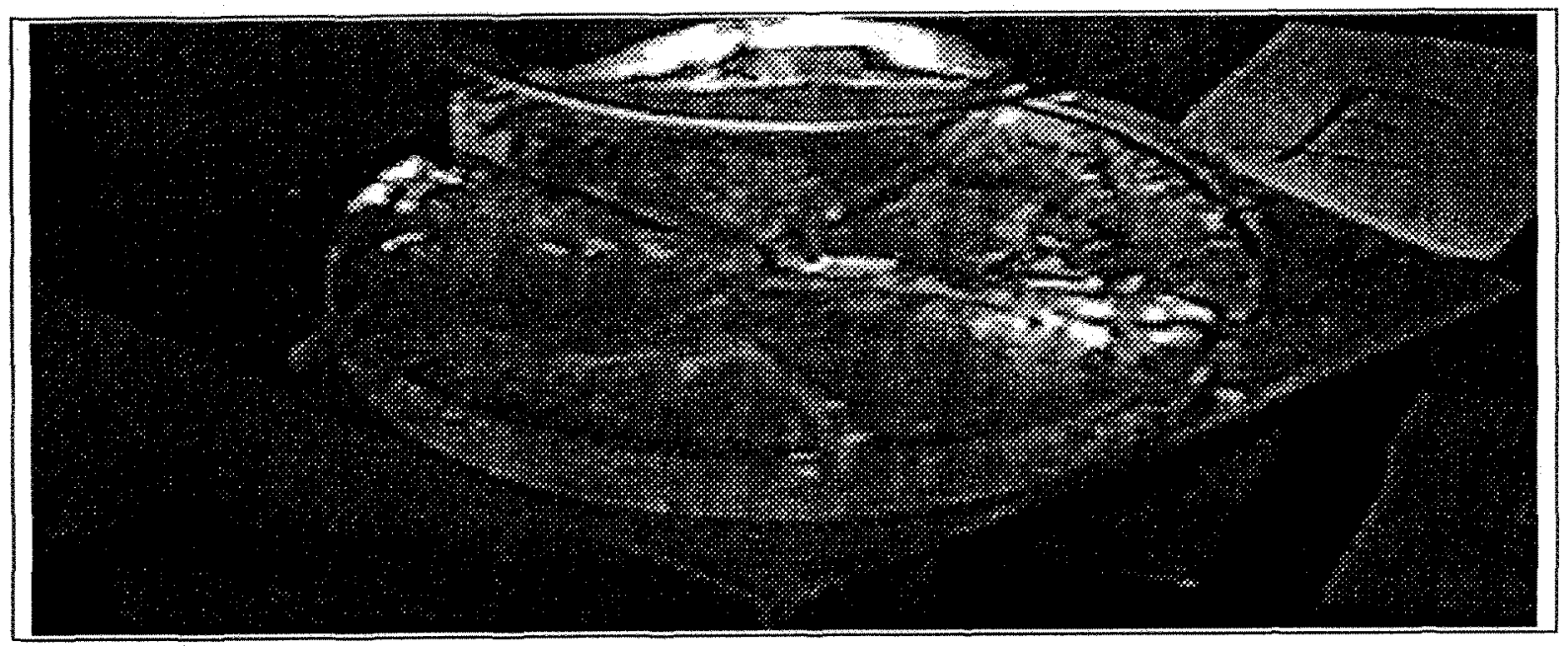

Figure 32: Échantillon de verglas d'une épaisseur 9,2 mm avec réflecteur 2 : troisième coup 
Finalement, le quatrième coup sépare complètement l'échantillon en cinq morceaux comme on peut le voir sur la figure 33 .

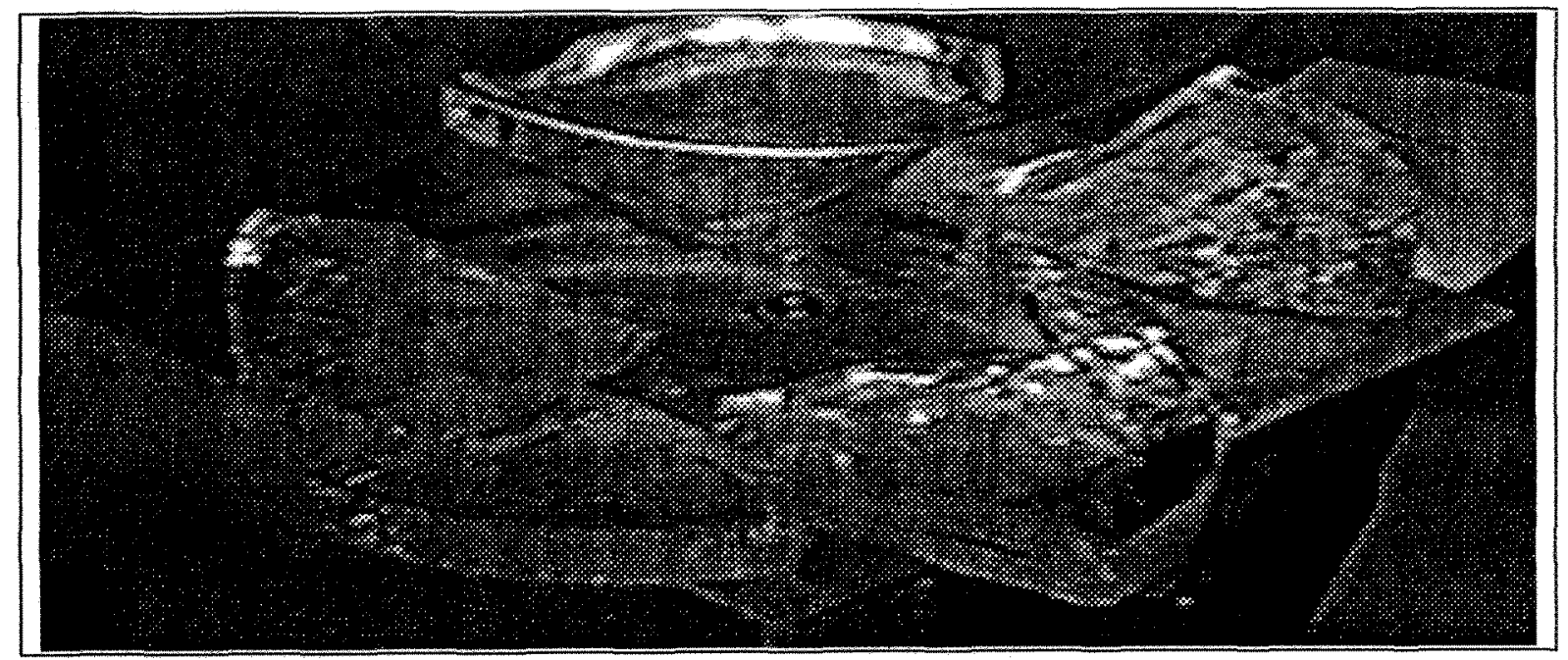

Figure 33: Échantillon de verglas d'une épaisseur $9,2 \mathrm{~mm}$ avec réflecteur 2 : quatrième coup 
L'échantillon suivant est d'une épaisseur de $9,9 \mathrm{~mm}$ La figure 34 nous montre celui-ci avant le passage de l'onde de choc focalisée par le réflecteur 3.

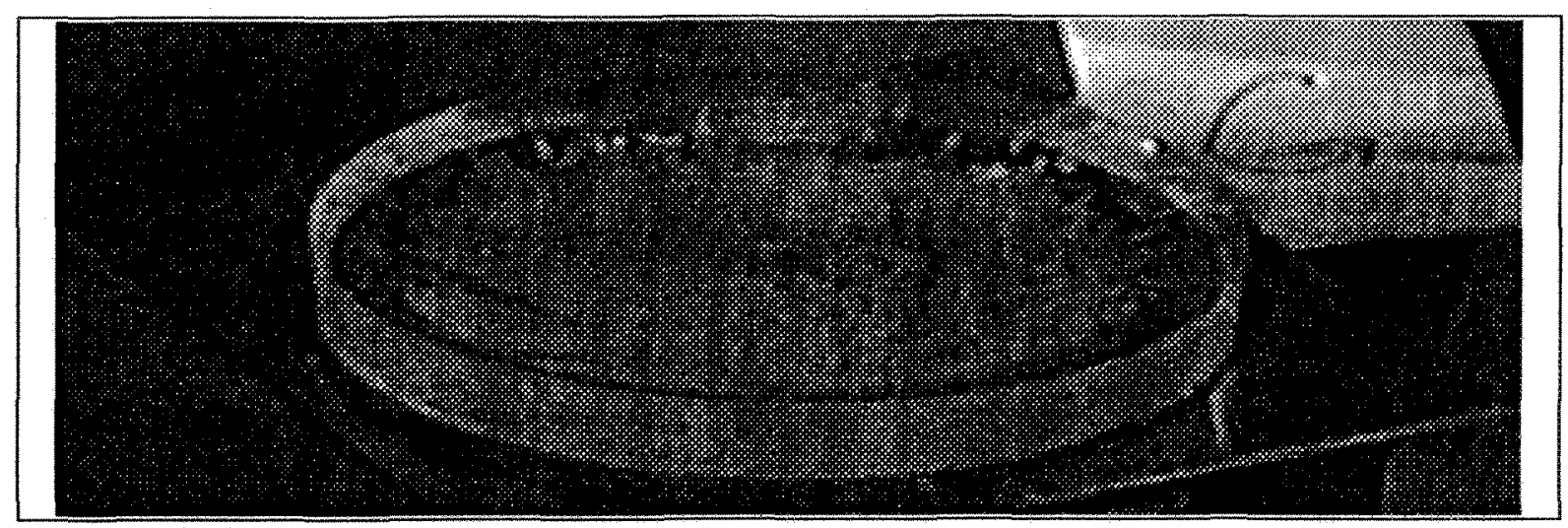

Figure 34: Échantillon de verglas d'une épaisseur $9,9 \mathrm{~mm}$ avec réflecteur 3 : aucun coup

Sur la figure 35 on distingue (voir pointillé) l'apparition d'une fissure de $4 \mathrm{~cm}$ au centre du disque après le passage d'une onde de choc. Vingt coups supplémentaires ont été appliqués sur cet échantillon et il n'y a eu aucune propagation de la fissure ni aucune apparition d'une autre fissure.

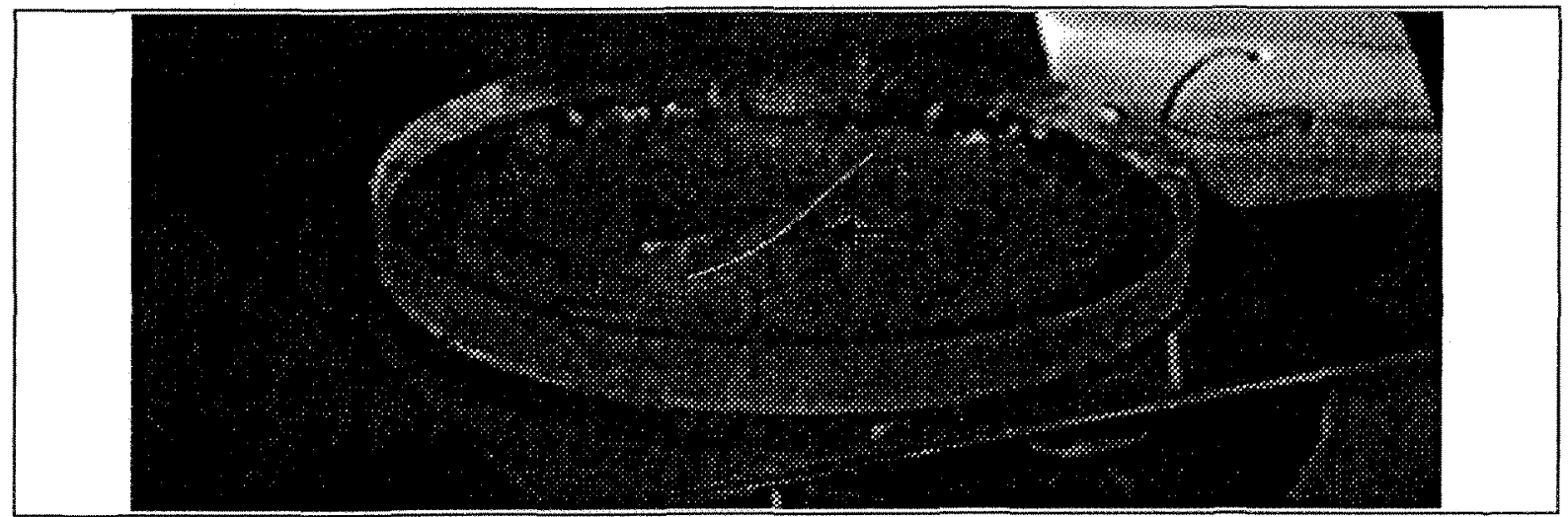

Figure 35: Échantillon de verglas d'une épaisseur $9,9 \mathrm{~mm}$ avec réflecteur $3:$ premier coup 


\subsection{Résultats des mesures d'impact avec une bille}

Dans la figure 36, l'axe vertical représente l'énergie potentielle gravitationnelle exprimée en Joule et l'axe horizontal représente la masse des échantillons en gramme. Cette façon, d'exprimer les résultats, permet de calculer l'énergie par unité de masse de glace nécessaire à la fracture.

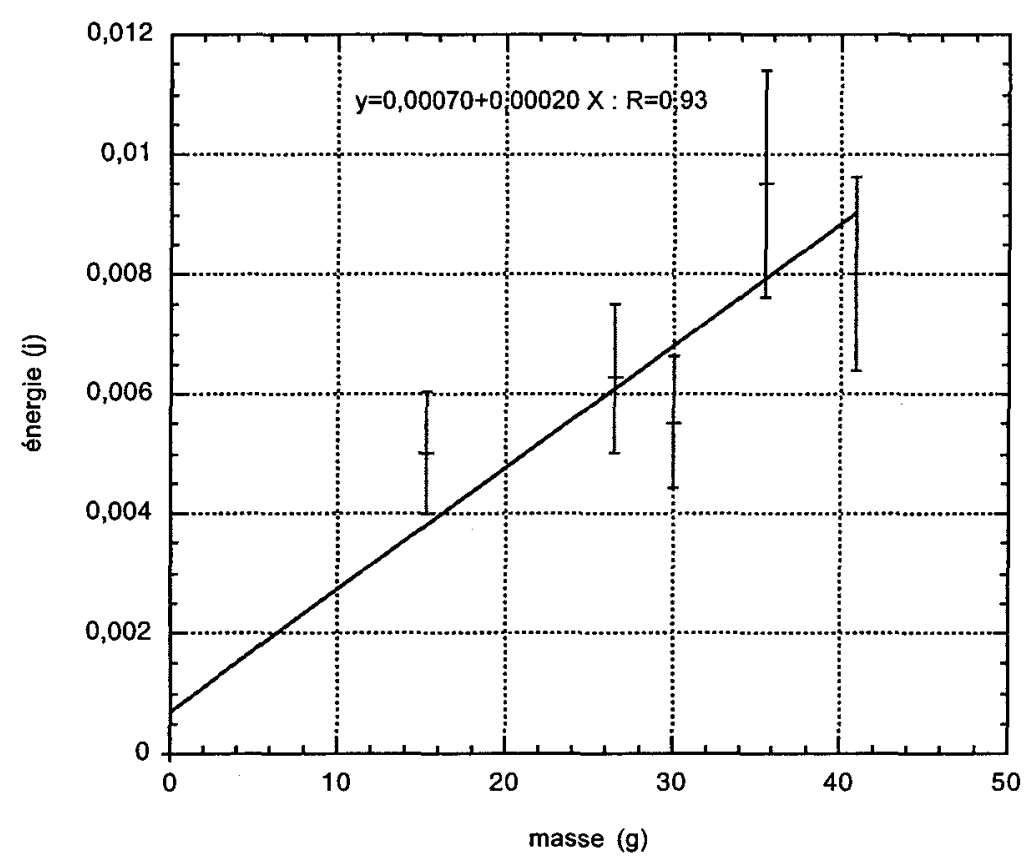

Figure 36: Résultats des essais de bris des échantillons de glace avec la bille. 
L'équation de progression linéaire permet d'obtenir une valeur d'environ $0,20 \mathrm{~J} / \mathrm{kg}$. Cette valeur est du même ordre de grandeur que celle mesurée par Laforte (1994) qui est de $0,25 \mathrm{~J} / \mathrm{kg}$ pour du verglas à $-2{ }^{\circ} \mathrm{C}$. Compte tenu du caractère destructif des essais, cette valeur est acceptable et reste dans les marges d'erreurs de l'expérimentation qui sont d'environ $20 \%$.

L'appareil brise, d'un seul coup, un échantillon d'une épaisseur maximale d'environ $9 \mathrm{~mm}$ qui a une masse de $44,9 \mathrm{~g}$. L'énergie nécéssaire pour briser cet échantillon est de :

$44,9 \mathrm{~g} \times 0,2 \mathrm{~J} / 1000 \mathrm{~g}=0,009 \mathrm{~J}$

Sachant que l'appareil consomme $50 \mathrm{~J}$ par coup ont obtient un rendement de :

$100 \times 0,009 \mathrm{~J} / 50 \mathrm{~J}=0,018 \%$ 
Chapitre 5

Discussion 


\section{$5 \quad$ Discussion}

Tout d'abord, on remarque que les échantillons réagissent différemment à l'onde de choc en fonction de leurs épaisseurs. Les échantillons dont l'épaisseur est inférieure à $9 \mathrm{~mm}$ se fracturent en plusieurs morceaux, de façon nette, après un seul passage de l'onde de choc, les échantillons dont l'épaisseur se situe entre 9 et $10 \mathrm{~mm}$ se fracturent après plusieurs coups et finalement ceux dont l'épaisseur est supérieure à $10 \mathrm{~mm}$ ne se fracturent pas.

Étant donné que l'on ne possède pas d'informations sur la partie de l'onde de choc effectivement transmise aux échantillons de glace, mais que l'on sait que la distribution de pression appliquée sur les échantillons ne sera pas toute captée par ceux-ci. Pour les besoins des calculs, on doit définir une distribution théorique de pression effective qui sera captée par l'échantillon. Pour ce faire, on a supposé une distribution de pression effective, cette distribution est symétrique selon le rayon de l'échantillon. Le maximum est au centre $(r=0)$ et possède une valeur d'environ $50 \%$ de la pression expérimentale maximale soit $175 \mathrm{kPa}$ par la suite, la pression décroît linéairement jusqu'à $0 \mathrm{kPa}$ sur le rebord de l'échantillon $(r=4,5$ cm). (voir Figure 37). 
Si cette solution n'est pas unique, elle a l'avantage d'être simple et de produire des résultats comparables à ceux qui sont obtenus lors de l'expérimentation.

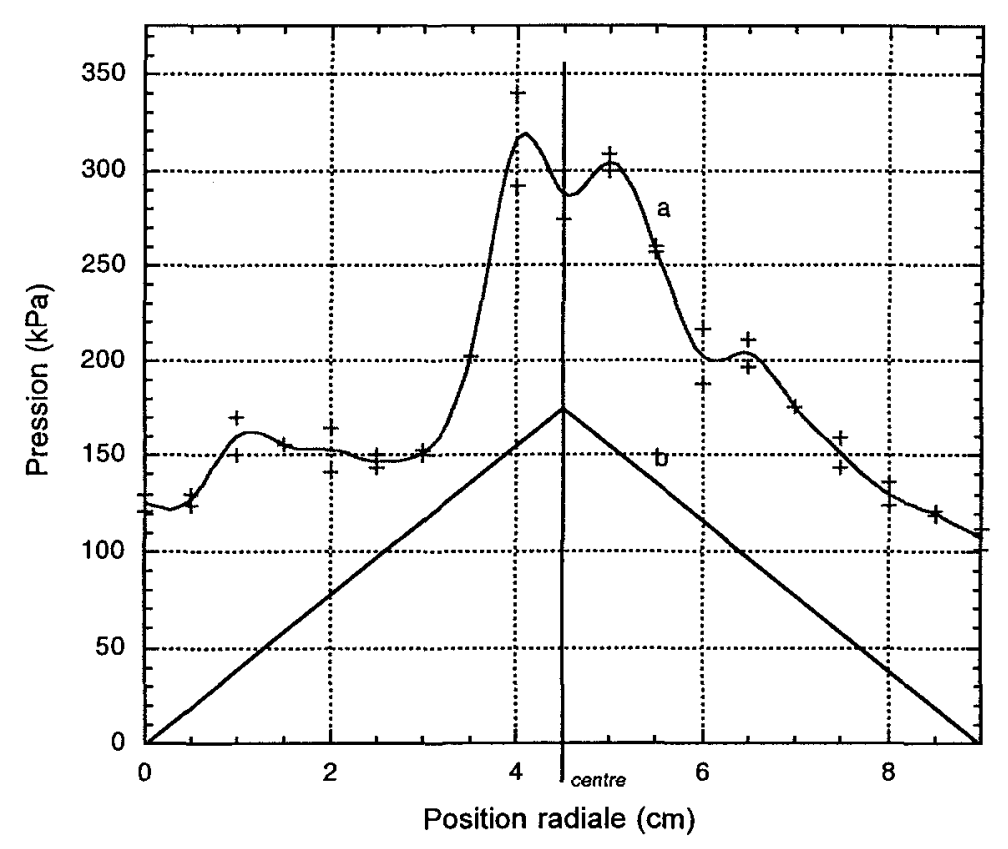

Figure 37: Comparaison entre le profil radial expérimental (a) et le profil théorique effectif (b).

Cette distribution de pression théorique a été utilisée pour calculer, à l'aide du modèle de disque en flexion développé à la section 2.5 .3 , la valeur des 
contraintes maximales au centre de la plaque pour des épaisseurs variant de 3 à $11 \mathrm{~mm}$. La figure 38 montre les résultats des calculs. On y retrouve la valeur des contraintes maximales évaluées au centre de la plaque en fonction de différentes épaisseurs $(3<\mathrm{h}<11 \mathrm{~mm})$, et la valeur de la contrainte limite de fracture $\sigma_{\mathrm{fr}}$ de la glace $\left(2,1 \times 10^{6} \mathrm{~N} / \mathrm{m}^{2}\right)$. On remarque que la limite de la contrainte de fracture est atteinte pour une épaisseur de $10 \mathrm{~mm}$ de glace avec ce type d'échantillon. Ce qui correspond aux résultats observés.

Le modèle prévoit aussi que les fractures seront orientées dans le sens radial ce l'échantillon. Ce qui correspond aussi au sens des fractures observées. Finalement, les essais produits sur des échantillons collés à la surface en aluminium du support n'ont présenté aucune fracture. En fait, toutes les observations et tous les calculs confirment que la fracture des échantillons de glace se produit en flexion. 


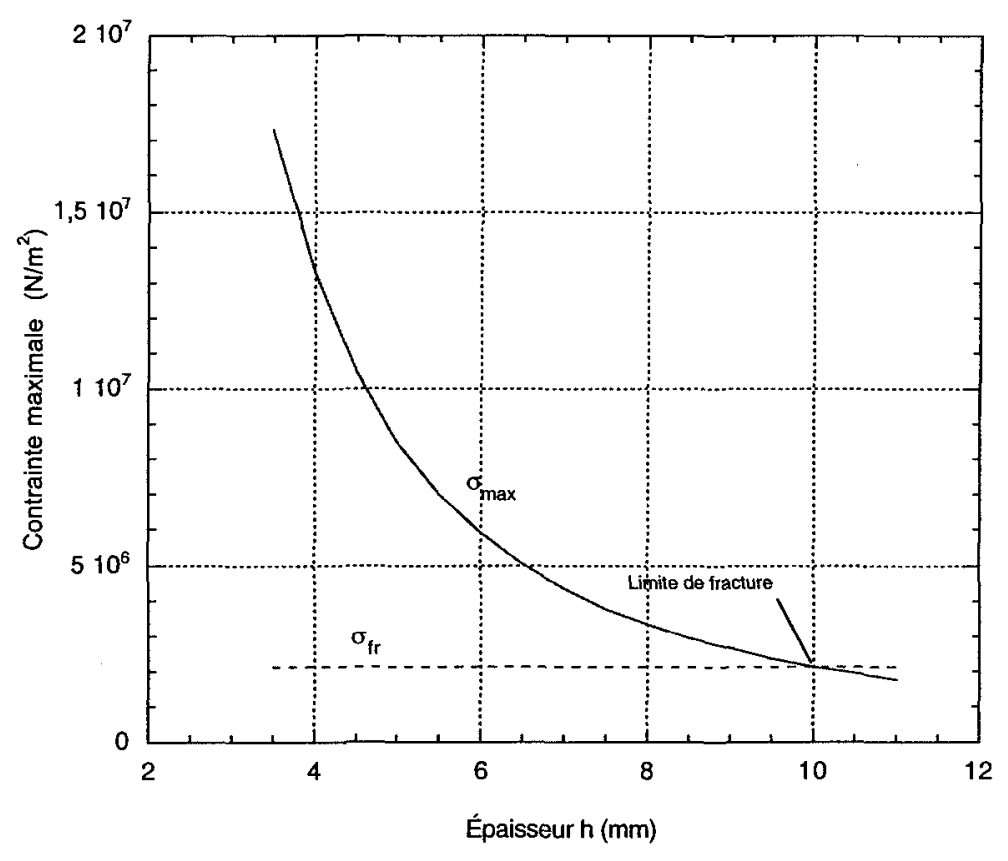

Figure 38: Contraintes maximales théoriques au centre du disque en fonction de l'épaisseur

Pour interpréter le processus de fracture pour les épaisseurs variant entre 9 et $10 \mathrm{~mm}$ où la fracture complète de l'échantillon se produit par une succession de coups, il faut se placer du point de vue énergétique. Pour produire une fissure en tension dans un matériau, il faut fournir l'énergie nécessaire à la formation des deux surfaces de cette fissure. Dans les matériaux ductiles, il faut fournir une énergie supplémentaire pour que cette fissure se propage alors que dans les 
matériaux fragiles la rupture se produit de façon catastrophique à une vitesse comparable à celle du son dans le matériau.

La glace sous le chargement rapide de l'onde de choc se comporte de façon fragile, la fissure devrait donc se propager de façon catastrophique. Mais ce n'est pas ce qui se produit avec les échantillons entre 9 et $10 \mathrm{~mm}$, c'est-à-dire ceux qui sont près de la limite de fracture. Ces observations conduisent à proposer une hypothèse qualitative du phénomène qui explique l'arrêt de la propagation des fissures dans l'échantillon.

Les échantillons ne sont pas des matériaux homogènes, car ils contiennent des bulles d'environ $0,1 \mathrm{~mm}$ de diamètre. Étant donné la façon dont les échantillons se sont formés, les bulles ne sont pas réparties de façon uniforme lors de la congélation. En fait, ces bulles sont formées à partir de l'air qui est dissous dans l'eau et qui est rejeté sous forme de bulles lors de la solidification. La taille et la position des bulles dans l'échantillon dépendent de la vitesse de solidification et de la direction selon laquelle la chaleur s'évacue. Dans le cas présent, on a fait congeler les échantillons dans des plats de Petri de $8,5 \mathrm{~cm}$ déposés sur une surface qui elle était déjà froide. L'air s'évacuait vers le haut de l'échantillon et l'évacuation de la chaleur se faisait à travers le fond du contenant. Dans ces conditions les bulles se retrouvent en plus grande quantité près de la surface de l'échantillon. 
La distribution des bulles est illustrée sur la Figure 39. On remarque qu'une mince partie de la section inférieure ne contient pas de bulles.

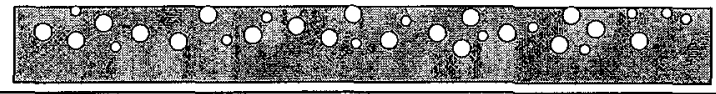

Figure 39: Échantillon de verglas avec bulles

Étant donné la répartition des contraintes dans l'échantillon, la fissure initiée en tension prend naissance au centre de la face inférieure du disque et se propage du centre vers le rebord et du bas vers le haut. La propagation de la fissure dépend du rayon de courbure de la tête de la fissure selon le mécanisme décrit par Griffith. La figure 40 illustre le mécanisme d'arrêt de la propagation. Lorsque la fissure rencontre une bulle la valeur du rayon de courbure est celui de la sphère, ce qui limite la concentration de contrainte et contribue à arrêter la progression de la fissure.

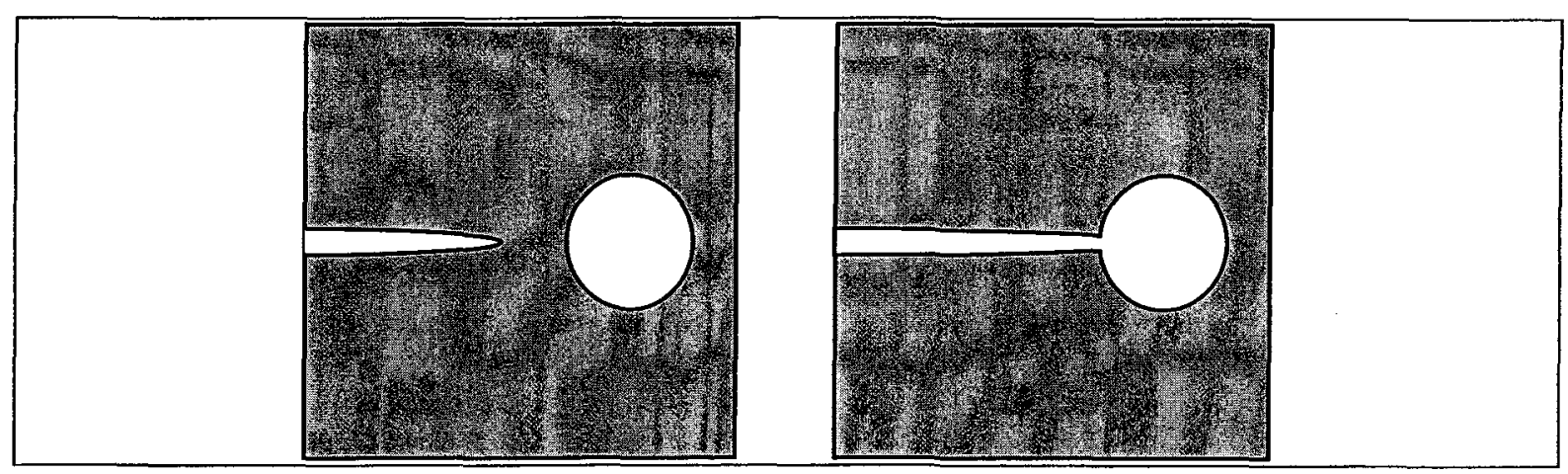


Figure 40: Mécanisme d'arrêt de la propagation de la fissure par une bulle.

Il faut donc ajouter à l'énergie nécessaire pour la production de la fissure une quantité d'énergie supplémentaire pour la propagation de la fissure. Cette hypothèse expliquerait le mécanisme d'arrêt de la propagation de la fissure dans les échantillons de glace et la nécessité de procéder par une répétition de coups pour obtenir la fracture complète de l'échantillon. Une expérimentation ultérieure d'ordre quantitative pourrait permettre de confirmer ou d'infirmer cette hypothèse. 
Chapitre 6

Conclusion 


\section{Conclusions et recommandations}

L'étude de la transmission d'une onde de choc au travers des échantillons de verglas a été effectuée et le théorème de l'énergie minimum a été utilisée avec succès pour calculer la limite de la fracture des échantillons en flexion. De plus, un modèle qualitatif du mécanisme de la propagation d'une fissure a été proposé. On peut conclure que :

1. Le montage expérimental a permis de produire une onde de choc présentant des pressions de $350 \mathrm{kPa}$ en pression positive et de -50 $\mathrm{kPa}$ en pression négative.

2. La transmission de l'onde de choc à l'interface air-glace brise, en flexion, les échantillons de verglas d'une épaisseur d'environ $9 \mathrm{~mm}$

3. Environ $50 \%$ de la distribution de pression a été effectivement captée par les échantillons contenant des bulles. Le rendement est de $1 \%$

4. L'énergie transmise est insuffisante pour provoquer la décohésion de la glace et par conséquent ne peut briser les échantillons collés sur une surface rigide.

5. Pour produire l'énergie nésséçaire au déglaçage d'une surface dans l'air, il faudrait dépasser la limite de résistance thermique des électrodes. 
On peut recommander que :

1. Sachant que les lithotriteurs qui fonctionnent en milieu liquide produisent des pressions positives de $40 \mathrm{MPa}$ et des pressions négatives de $10 \mathrm{MPa}$, il est raisonnable de croire que la modification de l'interface c'est-à-dire la transmission par contact, d'une onde de choc à partir d'un milieu liquide pourrait réussir à provoquer la décohésion de la glace.

2. L'utilisation d'un revêtement élastique possédant un large domaine élastique entre la glace et la surface, pourrait permettre le décollement et le bris de la glace par l'onde de choc.

Finalement, il serait souhaitable de poursuivre l'étude des ondes de choc autant du point de vue de la génération et de la transmission, non seulement dans la glace mais aussi dans les matériaux pouvant servir de revêtement. Car les méthodes de déglaçage mécaniques qui semblent être les plus efficaces font toujours appel à des déformations très rapides de la surface à déglacer qui entraînent le bris de la glace en régime fragile. 


\title{
Chapitre 7
}

\author{
Bibliographie
}




\section{$7 \quad$ Bibliographie}

ABEYARATNE ,R., KNOWLES, J.K., (2000) «A Phenomenological Model for Failure Waves in Glass ». Shock Waves, Vol. 10, pp. 301-305.

BOWER, A.F., (1998) « Advanced Mechanics of Solids » Lecture notes in Engineering175, Brown University.,[en ligne]. http://www.engin.brown.edu/courses/en175/notes.htm

BOWER, A.F., (1997) « Mechanics of Solids » Lecture notes in Engineering 222, Brown University, [en ligne].http://www.engin.brown.edu/courses/en222/notes.htm

BOWER, A.F., (1997) « Linear Elasticity « Lecture notes in Engineering 224, Brown University, ,[en ligne]. http://www.engin.brown.edu/courses/EN224/notes.htm

CLEVELAND, R.O., BAILEY, M.R., FINEBERG, N., HARTENBAUM, B., LOKHANDWALLA, M., MCATEER, J.A., STURTEVANT, B., (2000), « Design and Characterization of a Research Electrohydraulic Lithotripter Patterned after the Dormier Hm3 ». Review of Scientific Instruments.

QUIRK, J.J., KARNI S., On the Dynamics of a Shock-Bubble Interaction ». National Aeronautics and Space Administration, Report NAS1-19480. 
KITAGAWA, K., JYONOUCHI, T., YASUHARA, M., (2001) « Drag Difference between a Shocking Gas Flows passing through a Porous Body». Shock Waves, Vol. 11, pp. 133-139.

LAFORTE, J-L., (2000) «Thecnologie del'environnement nordique». Notes de cours, Université du Québec à Chicoutimi, Printemps, 332 p.

LAFORTE, J-L., ALLAIRE, M-A., ASSELIN, D., (1994) «État de l'art du déglaçage des conducteurs et des câbles aériens ». Groupe de Recherche en Ingénierie de l'environnement atmosphérique, Université du Québec à Chicoutimi, HydroQuébec, Décembre, 207 p.

LANDAU, L., LIFCHITZ, E., (1989). «Mécanique des fluides ».Physique théorique, Tome 6, Deuxième Édition. 752 p.

LEVY, A.,BEN-DOR, G., SKEWS, B.W., SOREK, S., (1993).« Head-On Collision of Normal Shock Waves with Rigid Porous Materials », Experiment in Fluid, SpringerVerlag.

LEVY, A., BEN-DOR, G., SOREK, S., (1998) « Numerical Investigation of the Propagation of Shock Waves in Rigid Porous Materials :Flow Field Behavior and Parametric Study ». Shock Waves), Vol 8, pp. 127-137.

LOKHANDWALLA, M., STURTEVANT, B., (2000)« Fracture Mechanics Model of Stone Comminution in ESWL and Implications for Tissue Damage ». Phys. Med. Biol., Vol. 45, pp. 1-18. 
MICHEL, B., (1978) «Ice Mechanics ». Les Presses de l'Université Laval, Québec, 484 p.

SCHEDIN, S., GREN, P.O., WAHLIN, A., (1997) « Shock Waves in Elliptic Cavity with Varying Height ». Shock Waves, Vol. 7, pp. 343-350.

STURTEVANT, B., (2000) «Shock Wave Lithotripsy » Graduate Aeronautical Laboratories,[en ligne], http://www.galcit.caltech.edu/ brad/bioscience/litho/index.html

WESTERMARK, S., NELSON, E., KINN, A.-C., WIKSELL, H., (1999) « The Impact of the Geometry of the Lithotriptor Aperture on Fragmentation Effect at Extracorporal Shock Wave Lithotripsy Treatment ». Urol. Res., Vol. 27, pp. 262-265.

YEW, C.H., WENG, X., (1987) «A Study of the Reflection And Refraction of Waves at the Interface of Water and Poro s Sea Ice ». J. Acoust. Soc. Am., (July), Vol. 82, pp 342-353. 


\section{Annexe 1}

Feuille de calcul sur Mathématica pour le calcul des contraintes en flexion des échantillons de glace 


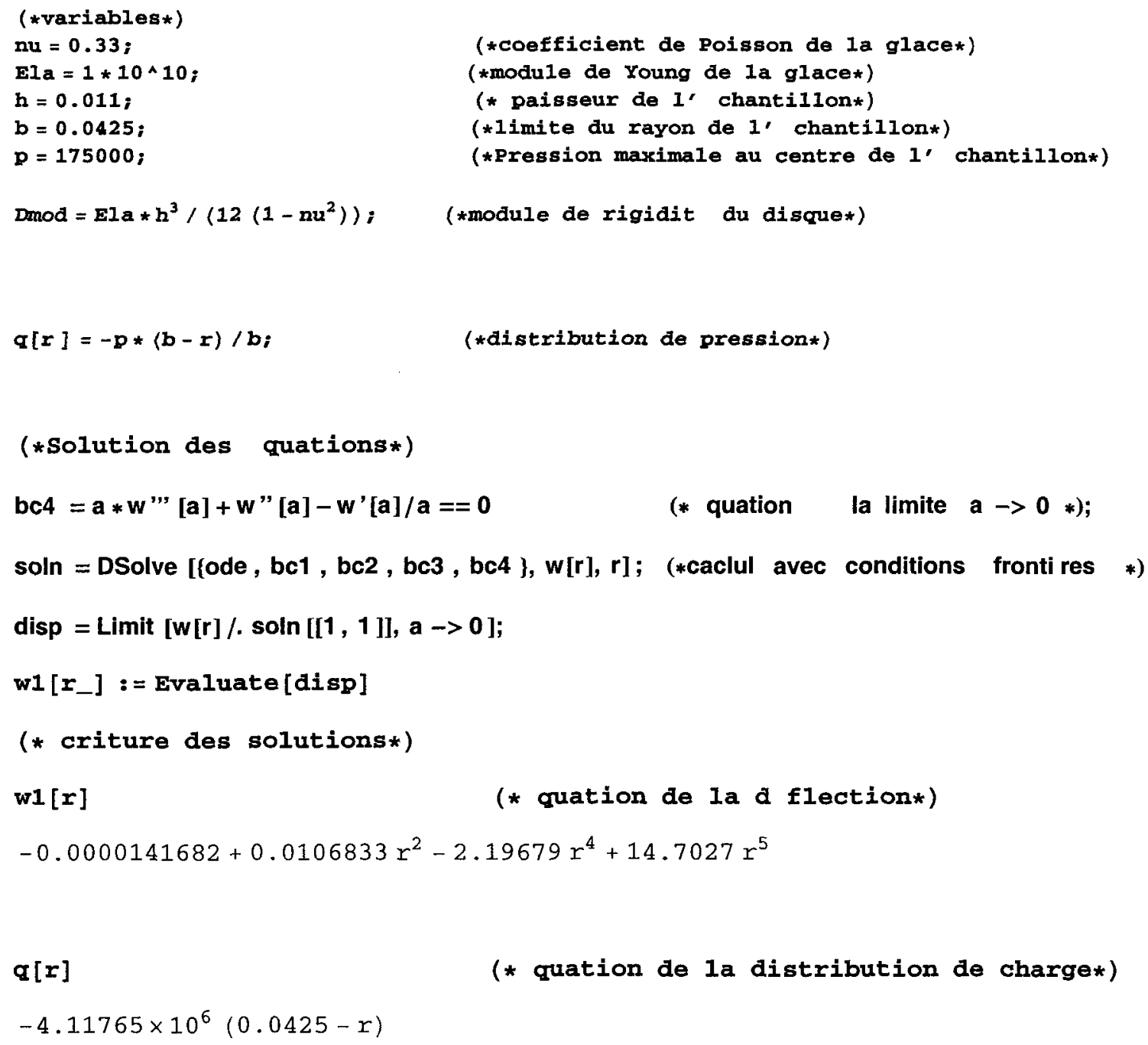


(*Calculs des contraintes*)

$\operatorname{sigmarr}\left[r_{-}, z_{-}\right]=\operatorname{simplify}\left[\operatorname{Ela} *\left(-w 1 *[r] * z-n u * w 1 \cdot[r] * \frac{z}{r}\right) /\left(1-n u^{2}\right)\right] ;$

sigmarr $[r,-h / 2]$;

sigmatheta $\left[r_{-}, z_{-}\right]=\operatorname{simplify}\left[\operatorname{Ela} *\left(-w 1,[r] * \frac{z}{r}-n u * w 1, '[r] * z\right) /\left(1-n u^{2}\right)\right]$;

sigmatheta $[r,-h / 2]$;

(*contrainte tengentielle $\sigma_{\theta \theta}$ valu au centre et sur la face in rieur de $1^{\prime}$ c) sigmatheta $[0,-h / 2]$

$1.75397 \times 10^{6}$

(*contrainte radiale $\sigma_{x r}$ valu au centre et sur la face in rieur de 1' chantil sigmarr $[0,-h / 2]$

$1.75397 \times 10^{6}$ 